\title{
Revealing the Solid-State Electrochemistry of Conjugated Oximates: Towards a New Functionality for Organic Batteries
}

Jiande Wang ( $\sim$ jiande.wang@uclouvain.be )

Université Catholique de Louvain

Petru Apostol ( $\square$ petru.apostol@uclouvain.be )

Université Catholique de Louvain

Darsi Rambabu ( $\sim$ rambabu.darsi@uclouvain.be)

Université Catholique de Louvain

Xuelian Liu ( $\nabla$ xuelian.liu@uclouvain.be)

Université Catholique de Louvain

Xiaolong Guo ( $\nabla$ xiaolong.guo@uclouvain.be )

Université Catholique de Louvain

Koen Robeyns ( $\nabla$ koen.robeyns@uclouvain.be )

UCLouvain https://orcid.org/0000-0002-4763-4217

Alae Eddine Lakraychi ( $\nabla$ alaeddinelakraychi@gmail.com )

Université Catholique de Louvain

Cristian Morari ( $\nabla$ cristim@itim-cj.ro )

Institutul Național de Cercetare-Dezvoltare pentru Tehnologii Izotopice și Moleculare Cluj-Napoca

Jean-Francois Gohy ( $\nabla$ jean-francois.gohy@uclouvain.be )

Universite catholique de Louvain

Deepak Gupta ( $\nabla$ deepak.gupta@uclouvain.be )

Université Catholique de Louvain

Alexandru Vlad ( $\square$ alexandru.vlad@uclouvain.be)

Université Catholique de Louvain https://orcid.org/0000-0002-0059-9119

Article

Keywords: Organic redox; Li-ion

DOI: https://doi.org/10.21203/rs.3.rs-1346580/v2

License: (c) (i) This work is licensed under a Creative Commons Attribution 4.0 International License.

Read Full License 
Additional Declarations: There is NO Competing Interest. 


\section{Revealing the Reversible Solid-State Electrochemistry of Lithium-Containing Conjugated Oximates: Towards a New Functionality for Organic Batteries}

Jiande Wang ${ }^{1, \S}$, Petru Apostol ${ }^{1, \S}$, Darsi Rambabu ${ }^{1, \S}$, Xiaolong Guo ${ }^{1, \S}$, Xuelian Liu ${ }^{1}$, Koen Robeyns ${ }^{1}$, Mengyuan Du ${ }^{1}$, Yan Zhang ${ }^{1,2}$, Shubhadeep Pal ${ }^{1}$, Robert Markowski ${ }^{1}$, Fabio Lucaccioni ${ }^{1}$, Alae Eddine Lakraychi ${ }^{1}$, Cristian Morari ${ }^{3}$, Jean-François Gohy ${ }^{1}$, Deepak Gupta ${ }^{1 *}$ and Alexandru Vlad ${ }^{1 *}$

${ }^{1}$ Institute of Condensed Matter and Nanosciences, Molecular Chemistry, Materials and Catalysis, Université catholique de Louvain, Louvain-la-Neuve B-1348, Belgium

${ }^{2}$ College of Materials Science and Engineering, Hunan Province Key Laboratory for Advanced Carbon Materials and Applied Technology, Hunan University, Changsha 410082, Hunan, P. R. China

${ }^{3}$ Institutul Național de Cercetare-Dezvoltare pentru Tehnologii Izotopice și Moleculare Cluj-Napoca, Cluj-Napoca, România

${ }^{\S}$ These authors contributed equally to this work;

Corresponding authors: deepak.gupta@uclouvain.be, alexandru.vlad@uclouvain.be

\section{Abstract}

In the rising advent of organic Li-ion positive electrode materials with increased energy content, chemistries with high redox potential and intrinsic oxidation stability, while in the lithium containing (Li-ion) form, remain a challenge. Molecular engineering of carbonyl compounds and of conjugated sulfonamide chemistries, have provided ground for future developments, while also inciting the search for other organic chemistry functions or classes, with alike properties, and with further improved energy storage metrics. Herein, we report on the solidphase reversible electrochemistry of a new class of organic redox functionality for batteries, the lithium salts of conjugated oximates. The disclosed first five examples of the oximate class, including cyclic (aromatic), and acyclic (non-aromatic), as well as aliphatic and tetra-functional stereotypes, uncover the complex interplay between the molecular structure and the electroactivity, as well as the potential of this rich family as Li-ion positive electrode materials for batteries. Amongst the exotic features specific to this class of materials, the most appealing one is the reversible electrochemical polymerization - depolymerization accompanying the charge storage process in solid phase, through intermolecular azodioxy coupling (-ONNO-) or the intramolecular furoxan cyclization of the oxidized forms. Over the disclosed materials, the best performing candidate delivers a high reversible capacity of $350 \mathrm{mAh} \mathrm{g}^{-1}$ at an average potential of $3.0 \mathrm{vs}$. Li $\mathrm{Li}^{+} \mathrm{Li}^{0}$, attaining $1 \mathrm{kWh} \mathrm{kg}^{-1}$ specific energy content at the material level metric. This work ascertains a strong link between the electrochemistry, organic chemistry and battery science by emphasizing on how different phases, mechanisms and performances can be accessed using a single chemical functionality. 


\section{Introduction}

Organic electrode materials are considered as one potential option for future electrochemical energy storage applications owing to appealing features of intrinsic natural abundance of the constituent elements, sustainability and low environmental footprint. ${ }^{1-4}$ The current battery value chain is exclusively dependent on transition metal electrode materials, which are procured after extensive mining and expensive synthesis protocols via energy consuming high temperature processing. Material costs, geopolitical issues related to sourcing of these, as well as handling and recycling encourage the efforts towards development of alternatives, amongst which organic electrode materials fulfill many conditions. The recent fast advancement of organic electrodes indicates that these may not only emerge as mere alternatives to the traditional transition metal positive electrode materials in conventional rechargeable batteries, but rather have the potential to lead to disruptive technologies. ${ }^{5}$

The charge storage mechanism of organic positive electrode materials can be divided into " $n$ type" or "p-type" redox systems. ${ }^{6,7}$ While the former have been studied mainly in their oxidized state (requiring battery discharge at first utilization, thus suitable only for the still under-developed lithium metal batteries), the latter stores the anion species, for application in dual-ion or anionic batteries. ${ }^{8}$ Conventional Li-ion cell assembly relies on a Li-ion source positive electrode material (the cathode) coupled to a Li-ion host material (the anode, in a rocking chair system ${ }^{9,10}$ ). Compared to the large diversity of inorganic Li-ion positive materials, the foundation of the state-of-the-art practical organic Li-ion positive electrode materials (mostly developed over the past 4 years) was laid extensively on enolate-carbonyl redox chemistry. Encouraging advancements have been attained through electronwithdrawing substituted quinones ${ }^{11}$, sacrificial metal-mediated charge delocalization ${ }^{12,13}$, and stereoelectronic chameleonic effect ${ }^{14}$. Recent advances have led to the development of a new class of organic redox systems for batteries, the conjugated sulfonamide chemistry with intrinsic ambient air stability while in the Li-reservoir form ${ }^{15}$. Whereas long considered challenging, the rationale of conjugated sulfonamides was based on decreasing the nucleophilicity of the organic anionic center, endowing resistance to hydrolysis as well as oxidation by molecular oxygen, while still allowing a reversible redox at a high potential. Unlocking the potential of this class of material not only provided new avenues for molecular engineering towards improved charge storage performances of also other monovalent cations, but also encouraged the idea of exploring other organic redox systems with alike properties $^{16,17}$. 
In this work, we present the first application of conjugated oximate Lithium salts as positive electrode materials for batteries. We analyze and discuss the rich physico-chemistry processes accompanying the electrochemistry in solid-phase of these materials. To establish the versatility of the oximate redox chemistry as electrode materials for Li-ion batteries, five lithiated oximate systems with different chemical structures, including cyclic (aromatic), acyclic (non-aromatic), aliphatic and tetrafunctional stereotypes, are investigated. Each system is found to evolve differently upon redox, with characteristic features originating from specific intermolecular and intramolecular interactions. Amongst the peculiar characteristics found, the most intriguing include the solid-to-liquid reversible conversion of dilithium dimethylglyoxime upon redox, and the reversible electrochemical polymerization - depolymerization accompanying the charge storage process in solid phase of the dilithium $p$-benzoquinone dioxime. The reversibility of the intermolecular azodioxy coupling (-ONNO-) or the intramolecular furoxan cyclization is also analyzed. The best performing candidate (dilithium $p$ benzoquinone dioxime) displays a high reversible capacity of $350 \mathrm{mAh} \mathrm{g}^{-1}$ at a redox potential of $3 \mathrm{~V}$ vs. $\mathrm{Li}^{+} / \mathrm{Li}^{\circ}$, reaching a specific energy density of nearly $1 \mathrm{kWh} \mathrm{Kg}^{-1}$, estimated at the material level. The excellent chemical and structural reversibility, as well as the promising electrochemical performances of the studied conjugated oximates corroborate the promising potential of this class of materials as positive electrode materials for Li-ion batteries. The oximate redox functionality not only enriches the family of organic electrode materials (Table S5), but also provides excellent redox reversibility, low molecular weight (equivalent of high capacity) and dry air-stability of the anionic form, fulfilling the requirement of positive electrodes as new candidates for Li-ion batteries.

\section{Design rationale and redox mechanisms of conjugated oximates}

The skeleton of oximate redox functionality exemplifies the fusion of two important families of organic electrochemical storage mechanisms, namely the conjugated carbonyls with the nitroxides (Fig. 1a). The conjugated carbonyl system ( $n$-type) remains probably the most investigated, and dominated the organic battery field with more than 100 structural analogues reported to date ${ }^{18}$. The nitroxide redox ( $p$-type), initially reported by Nishide, Oyaizu and co-workers, has attracted rapid attention in the community given the high redox potential (3.6V vs. $\mathrm{Li}^{+} / \mathrm{Li}, p$-type, anion storage) and the large versatility in incorporating this redox center into polymeric or small molecular systems. ${ }^{19,20}$ 
The reduced form of quinones (enolates) takes advantage of $\pi$-conjugation (aromatic system) to achieve stabilization through the mesomeric effect, ${ }^{11,14,21}$ whereas the nitroxide radical is an ambipolar redox center; with the nitroxide - oxoammonium $p$-type redox couple $\left(3.6 \mathrm{~V} \mathrm{vs}\right.$. $\left.\mathrm{Li}^{+} / \mathrm{Li}\right)$ being well studied, whereas the nitroxide - aminoxyl anion $n$-type redox couple $\left(2.8-3.0 \mathrm{~V} \mathrm{vs}\right.$. $\left.\mathrm{Li}^{+} / \mathrm{Li}\right)$ remaining elusive and less-understood. ${ }^{7,22,23}$ Thus, the amalgamation of the concepts of charge stabilization by conjugation with the nitroxide-based localized redox would potentially result in a nitroxide bearing conjugated $\pi$-system (e.g. pyrazine- $N, N^{\prime}$-dioxide) that might significantly favor radical stability and redox kinetics, although such a molecular system remains elusive so far (Fig. 1a, right-hand panel).

a

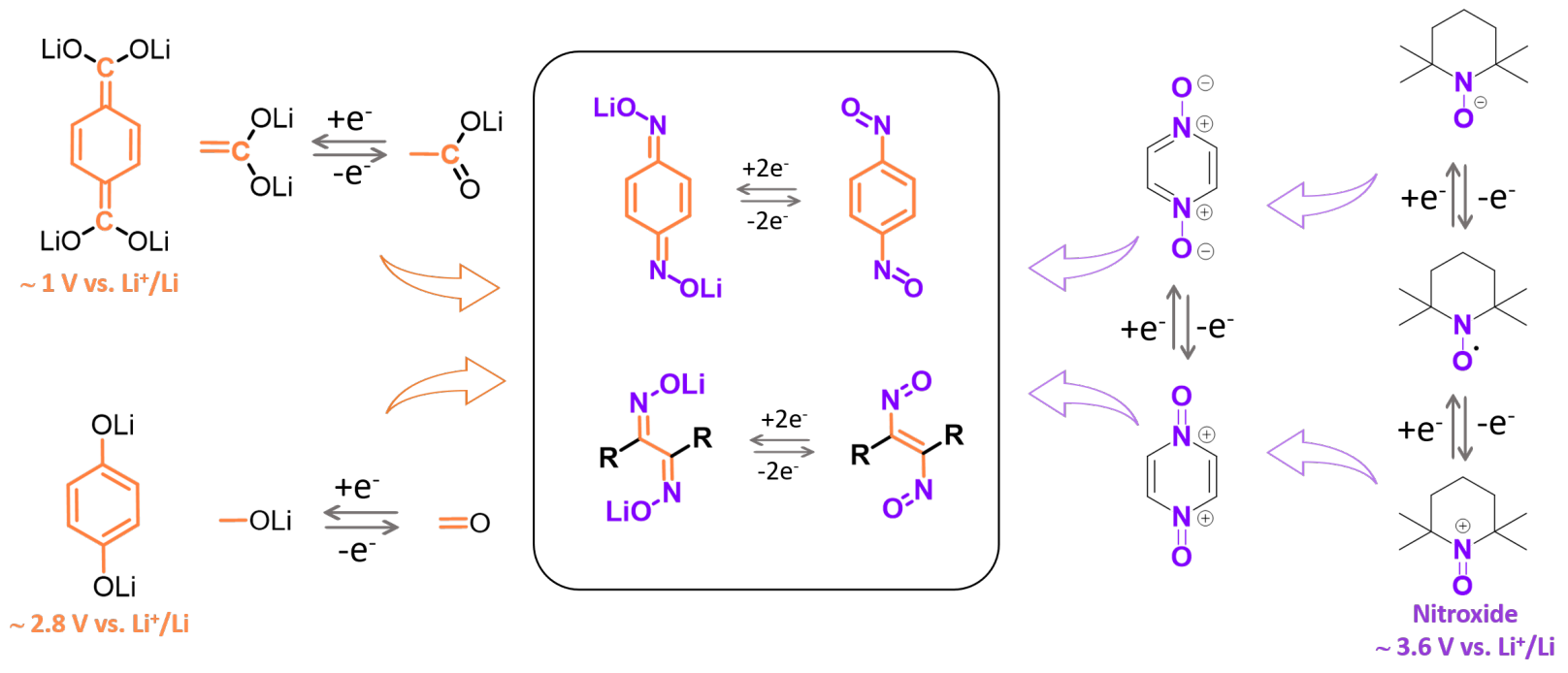

b

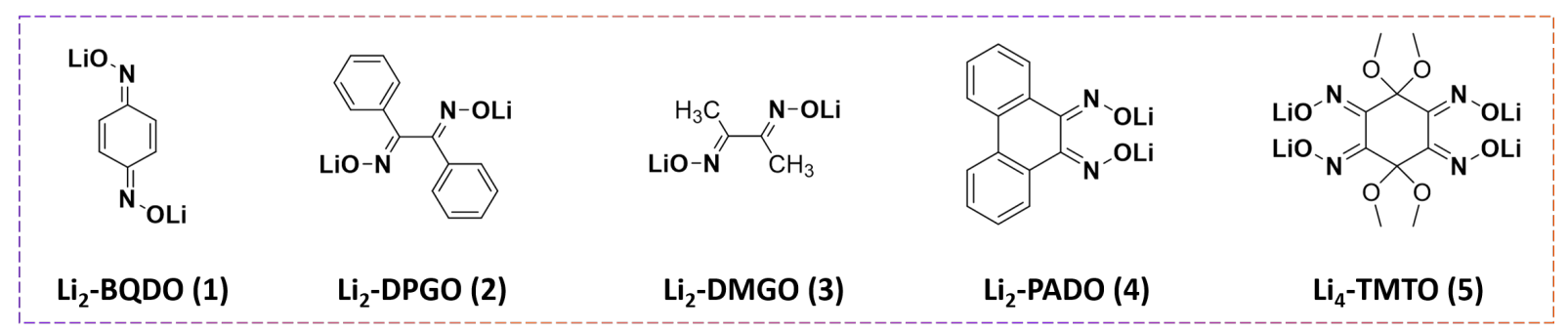

Figure 1. Design rationale and the library of conjugated oximates. a) Left: Conjugated carboxylate and ketoenolate redox. Right: Nitroxide-based redox (oxoammonium cation $\leftrightarrow$ nitroxide $\leftrightarrow$ aminoxy anion). Molecular design of conjugated oximates (center) can be regarded as intramolecular hybrid fusion of di-aminoxy (right) and quinoneimine functions (left). b) The first generation of conjugated oximate cathode materials disclosed in this work and the respective abbreviations used. 
On the other hand, in conjugated dicarboxylate systems, the redox potential of the carbonyl group is significantly lower (in the range of $1 \mathrm{~V} \mathrm{vs}$. $\mathrm{Li}^{+} / \mathrm{Li}$, as compared to $2.0-3.0 \mathrm{~V} \mathrm{vs}$. $\mathrm{Li}^{+} / \mathrm{Li}$ found in most carbonyl derivatives) owing to the strong electron donating effect of the $-\mathrm{OLi}$ attached to the $\mathrm{C}=\mathrm{O}$ carbon (the resonance carboxylate group), making these suitable as negative electrode materials for Liion storage (Fig. 1a, left panel). ${ }^{19}$ We envisioned that the rationale of eschewing electron-donating group (-OLi) is a prerequisite for augmenting the redox potential, which would be possible by replacing the tetravalent carbon unit with a trivalent nitrogen atom, leading to the ketoximate group $\left(=\mathrm{N}-\mathrm{O}^{-}\right)$that would merge the essential features of nitroxide and carbonyl redox moieties. The benzoquinone dioximate (Fig. 1b (1), BQDO ${ }^{2-}$ ) represents thus a hybrid of iminoxy group and quinoneimine center with a reversible two-electron redox process, that was also found to be accompanied by a series of exotic processes, depending on the position of redox functionality and conjugation as discussed in the following sections. As the conjugated non-aromatic redox systems remain scarcely studied for organic battery electrode materials, a series of additional oximate derivatives (e.g., lithium salts of DPGO ${ }^{2-}(2)$, $\mathrm{DMGO}^{2-}(3), \mathrm{PADO}^{2-}(4)$ and $\mathrm{TMTO}^{4-}(5)$ - displayed in Fig. 1b) drew our attention as model compounds, due to their rich chemistry as well as low molecular weight, translating to design diversity and potentially high theoretical capacities (Fig. 1b).

All lithium salt of studied oximate anions (1-5) were prepared by direct acid-base reaction on the protonated versions at room temperature (refer to Experimental Section in SI: materials synthesis). Complete deprotonation was qualitatively confirmed by the disappearance of the $3200 \mathrm{~cm}^{-1}$ band of the hydroxyl group in FTIR analysis (Fig. S14-S18). Being aware of the fact that air-stability of cathode materials is a crucial parameter for practical applications and only a handful of organic positive Li-ion electrode materials comply to this requirement thus far ${ }^{11,12,14,15}$, we focused our initial attention to the reactivity of the lithiated phases in ambient and controlled atmospheric environment. All the studied oximate (1-5) materials displayed no significant chemical changes (asserted by FTIR analysis) after 48 hours of exposure to dry air, confirming the excellent oxidation stability of the oximate lithium salts (refer to Fig. S14-S18 and associated text for details on measurements and analysis). 


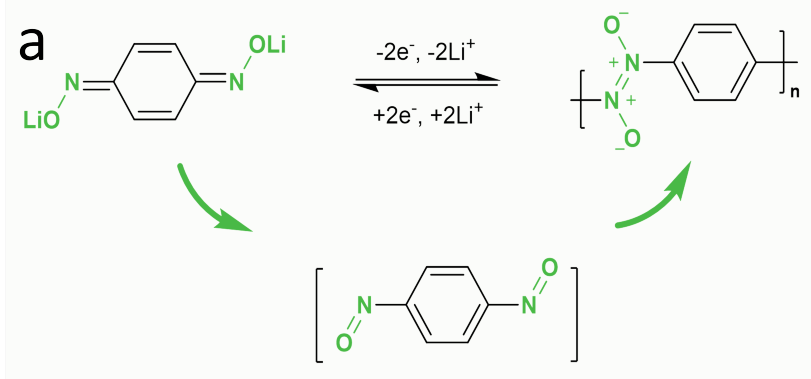

Mechanism 1 intermolecular coupling
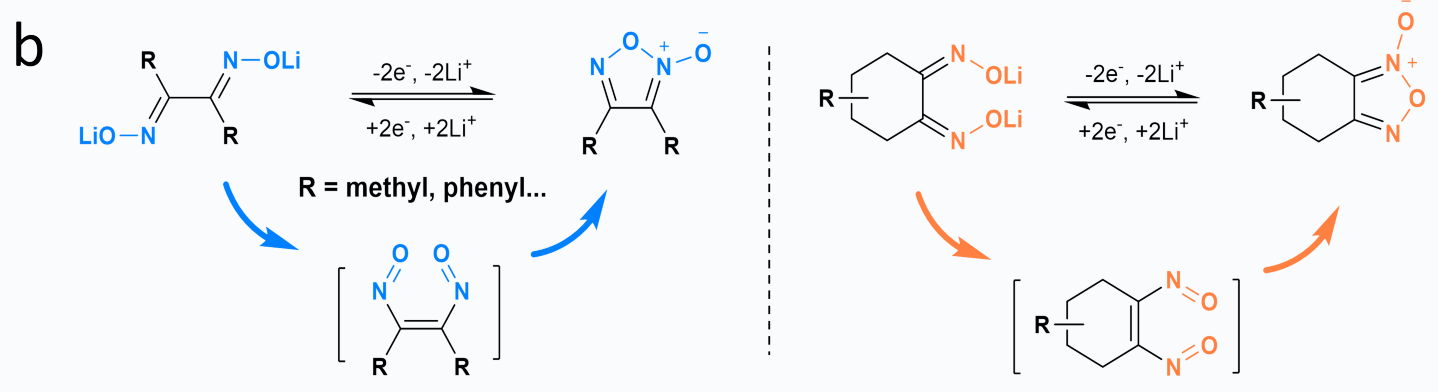

Mechanism 2 intramolecular cyclization

Figure 2. Reversible intra- and inter-molecular conversion during redox in lithium oximates. (a) Redox mechanism of $L i_{2}-B Q D O$ : upon oxidation, $L i_{2}-B Q D O$ exchanges two electrons and lithium ions forming the intermediate para-dinitrosobenzene, which undergoes intermolecular coupling to form PNND through azodioxy (-ONNO-) linkage. (b) left panel: Redox mechanism of 1,2-oximates ( $\mathrm{Li}_{2}-D P G O$ and $\mathrm{Li}_{2}$-DMGO): after oxidation, the dinitroso intermediate forms the furoxan ring via intramolecular cyclization; right panel: Redox mechanism of ortho-oximates ( $\alpha$-dioximates) showing the formation of furoxan derivatives as the oxidation products.

The redox chemistry of oximes is relatively well studied, with primary interest on the anodically generated nitroso form $(-\mathrm{N}=\mathrm{O})$ that has industrial as well as fundamental considerations ${ }^{24-27}$. Most of the organic nitroso compounds have been found to behave differently in dissolved and solid phases (for example, displaying pale yellow coloration in the solid state, as compared to bright green in solution $)^{28}$. This was attributed to the reversible dimerization of the nitroso compounds, existing as monomers in solution, and dimeric in the solid state, at room temperature. The (di)nitroso intermediates are indeed stable only at low temperatures $(<130 \mathrm{~K})$ or elevated temperature in gas phase, with ability to dimerize or polymerize spontaneously at room temperature ${ }^{29}$. The reversible dimerization of nitroso derivatives leads to the formation cis or trans azodioxides, or furoxan derivatives, 
depending on the electronic conjugation as well as molecular structure. The activation energy for the dissociation of azodioxy dimers is in the range of 20-30 $\mathrm{kcal} \mathrm{mol}^{-1}$, suggesting that this covalent bond is strong, but can nevertheless be broken and reformed reversibly under mild conditions. This aspect has been for example ingeniously exploited for the construction of monocrystalline covalent organic networks with a variety of linkers and topologies. ${ }^{24}$

However, despite the advances in understanding and application of nitroso-azidioxy chemistry, some fundamental differences, as well as open questions and contradictions remain. Most studies have been performed so far on the dissolved phase of the protonated oximate forms, with sole emphasis on the chemical oxidation process. While the reversibility of the nitroso - azidoxy polymerization depolymerization process is relatively well understood, the reversibility of the redox processes (either by chemical or electrochemical means) remains seldomly addressed and contradictory. This is an essential aspect of these materials to investigate, given the complex intra- and inter-molecular conversion taking place (Fig. 2, and discussed further), that could be intuitively treated as irreversible. For example, some quinone dioximes were reported as irreversible, with only dimethyl ether derivatives showing reversible redox, yet proceeding through a $p$-type mechanism (formation of radical cation upon oxidation) ${ }^{26}$. Our findings not only confirm the reversibility of the redox, but also the reversibility of the subsequent dimerization-polymerization processes in the solid phase, beginning with either anionic (reduced) or the oxidized forms (cation-electron coupled events). All disclosed oximate lithium salts (1-5) were found to undergo a reversible one-electron per oximate group redox, forming the intermediate nitroso derivatives, followed by significant reversible structural modifications, which are strongly dependent on electronic conjugation and molecular structure (Fig. 2). For instance, the electrochemical oxidation product of $\mathrm{Li}_{2}-\mathrm{BQDO}$ was identified as poly $(1,4-$ phenyleneazine- $E-\mathrm{N}, \mathrm{N}-$ dioxide) (PNND, Mechanism 1), similar to the one obtained by chemical oxidation of p-benzoquinone dioxime with an excess of sodium hypochlorite in alkaline aqueous medium ${ }^{29,30}$.

The in-situ formation of PNND upon oxidation of $\mathrm{Li}_{2}-\mathrm{BQDO}$ was also confirmed by Rietveld refinement of the experimental PXRD data (Fig. S19 and S20). ${ }^{31}$ Only the product with E- (trans-) configuration around the $\mathrm{O}-\mathrm{N}=\mathrm{N}-\mathrm{O}$ groups was detected, as also confirmed by the absence of the characteristic FTIR band for Z- (cis) conformer in the range of $\left.1350-1400-\mathrm{cm}^{-1}\right)^{32,33}$. This is consistent with the previous study on kinetics and thermodynamic stability of the PNND de/polymerization 
process, with exclusive formation of the $E$-configuration at ambient temperatures ${ }^{25}$. The sequence of redox and de-polymerization steps in solid-phase was found to be highly reversible, while being also exploited and proving advantageous for Lithium storage. For instance, the PNND polymeric form was found to display low solubility in battery electrolytes (Table S6, Fig. S21), the soluble species being composed of monomer, or low-unit oligomers (and not the polymeric form), as a result of azodioxy nitroso equilibrium ${ }^{24}$ (refer to $\mathrm{SI}$ for additional details and discussion). The low solubility is an interesting feature for energy storage application applications, given the general problem of elution of organic electrode materials typically arising from the oxidized/neutral phase (e.g., benzoquinone), unlike the PNND which is poorly soluble in commercial electrolytes (Table S6, Fig. S21). ${ }^{34}$

The $\alpha$ - or 1,2-dioximates (e.g., $\mathrm{Li}_{2}$-DPGO, $\mathrm{Li}_{2}$-PADO and $\mathrm{Li}_{4}$-TMTO) also oxidize into the intermediate dinitroso states, which undergo intramolecular cyclization to furoxan derivatives as the final product (Fig. 2. Mechanism 2, e.g., 3,4-diphenyl-1,2,5-oxadiazole-2-oxide (DPODO) as the oxidized phase of $\mathrm{Li}_{2}$ DPGO). ${ }^{29,35,36}$ The redox of the linear aliphatic $\mathrm{Li}_{2}$-DMGO (3) proceeds through an additional unconventional solid-liquid conversion process, in that the oxidation reaction product (3,4-dimethyl furoxan) is liquid at ambient temperature, a feature that is highly suitable for a redox flow battery systems ${ }^{37,38}$. Interesting, despite the liquid phase of the end of charged product, the cell based on $\mathrm{Li}_{2-}$ DMGO displayed high material utilization and good electrochemical reversibility at the first charge discharge cycle (Fig. S27). The formation of furoxan ring in solid state upon charge-discharge influences

the redox process and changes the electrochemical performance of the electrode materials, which will be discussed in the next section.

\section{Solid-phase electrochemical and charge storage characteristics}

Having established the structural and electrochemical features of the oximate-[nitroso]-azodioxy such as reversible electrochemical and chemical conversion, the solid-state electrochemical performance of 1-5 as positive electrode materials was evaluated next (Fig. 3). All studied compounds display the theoretical one-electron redox per oximate group, with average redox potential spanning the range of 2.5-3.1 V vs. $\mathrm{Li}^{+} / \mathrm{Li}$, and efficient material utilization with high capacities of 210-350 mAh/g

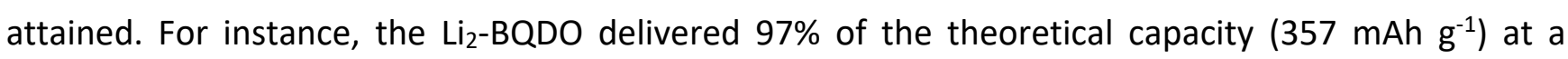
potential around $3.0 \mathrm{~V}$ vs. $\mathrm{Li}^{+} / \mathrm{Li}$ on the first cycle, corresponding to $1.94 \mathrm{Li}^{+} / \mathrm{e}^{-}$equivalents utilization (Fig. 3a, green curve, galvanostatic charge/discharge rate of one $\mathrm{Li}^{+}$ion in $5 \mathrm{~h}$ ). The cells also displayed 
good rate capability (Fig. S22), as well as stable behavior with more than $200 \mathrm{mAh} \mathrm{g}^{-1}$ retained after 50 cycles (over $75 \%$ of capacity retention, Fig. S23-S24). Whereas these performances certainly require additional electrode and electrolyte engineering to enhance the cycling stability and power performances, conceptually, it is interesting to compare the performances of $\mathrm{Li}_{2}-\mathrm{BQDO}$ to its closest analogue, the dilithium benzene-1,4-diolate ( $\left.\mathrm{Li}_{2}-\mathrm{BQ}\right)$. First, $\mathrm{Li}_{2}-\mathrm{BQDO}$ displays a higher redox potential $(\sim 300 \mathrm{mV})$ in both dissolved and solid forms ${ }^{14}$ (Fig. S26). This confirms the hypothesis proposed earlier that the induction of nitrogen between the hydroxyl redox center and the cyclohexadiene ring leads to enhanced electronic delocalization through resonance effects, as well as the redox potential increase. It is also worth noting that $\mathrm{Li}_{2}-\mathrm{BQ}$ can barely sustain few cycles in liquid electrolytes given the high solubility of the oxidized phase (1,4-benzoquinone) in polar solvents, whereas $\mathrm{Li}_{2}$-BQDO shows considerably ameliorated cycling stability, benefiting from the polymeric PNND phase impeding significant material dissolution. Replacing $\mathrm{Li}_{2}-\mathrm{BQDO}$ with PNND (prepared by chemical oxidation of $\mathrm{H}_{2}-$ BQDO) as active electrode material and starting the galvanostatic cycling in discharge (reduction) mode, similar galvanostatic profiles were attained (Fig. 3a - black curve, and Fig. S25), additionally confirming that PNND polymer is indeed the electrochemical oxidation product.
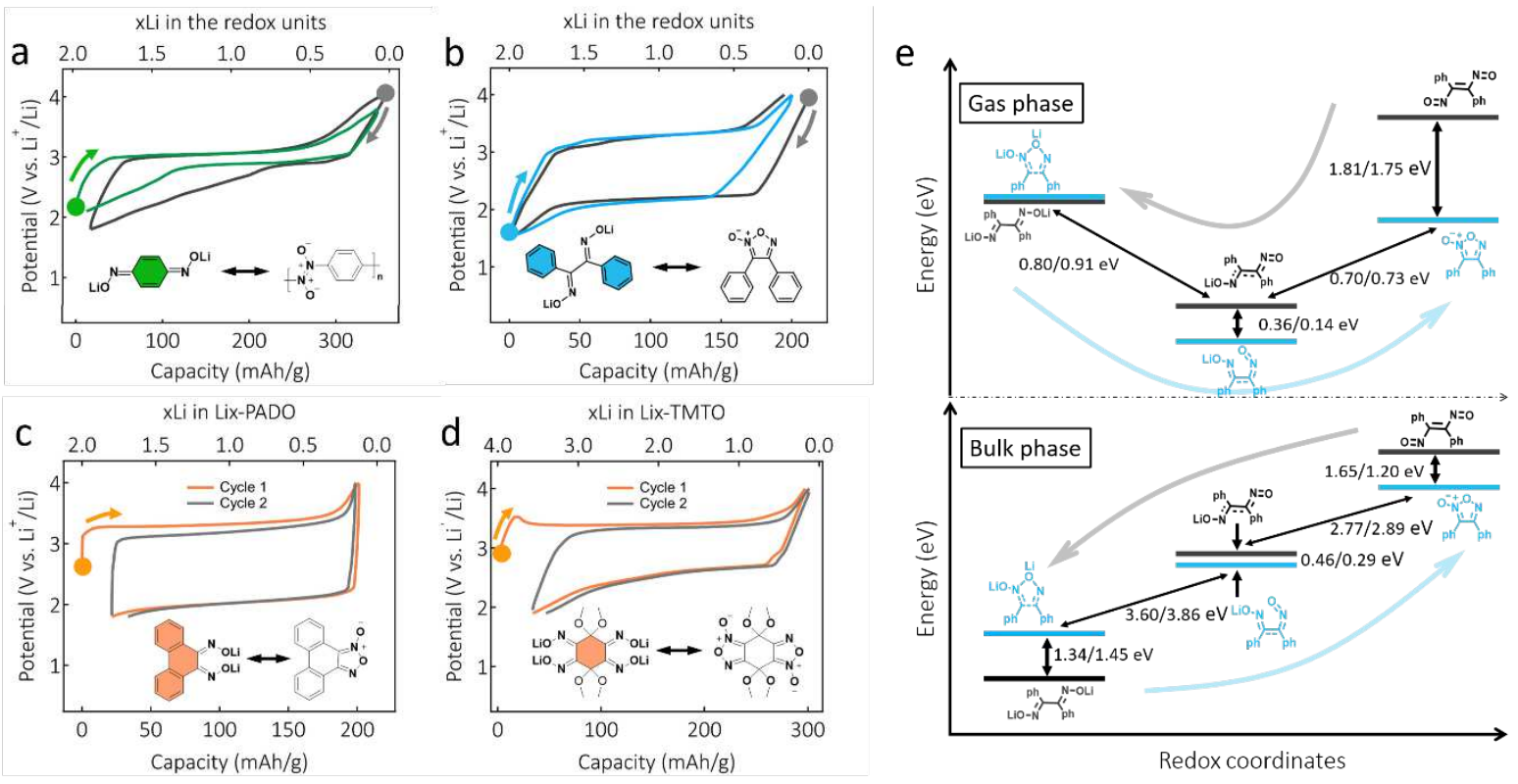

Figure 3. Solid-state electrochemistry of lithium oximates $(1,2,4$, and 5) as positive electrode material for charge storage. Potential-composition galvanostatic charge-discharge profiles of (a) Li $i_{2}-B Q D O$ (green curve) and PNND (gray curve) electrode materials cycled at a rate of $\mathrm{C} / 10$ (equivalent of $1 \mathrm{e}^{-} / 1 \mathrm{Li}^{+}$exchanged in 5 hours), (b) Li $i_{2}$-DPGO (blue curve) and DPODO cells (black curve) electrode material, (c) Li $i_{2}$-PADO electrode material, and (d) of $\mathrm{Li}_{4}$-TMTO electrode material. (e) Redox coordinates for the conversion of $\mathrm{Li}_{2}$ - 
DPGO to DPODO, considering reaction pathways for the linear and cyclic configurations, in both gas and solid phase (refer to DFT Calculation Section in supporting information document). The energy differences for phase transition (eV) are indicated for both experimental and calculated cell parameters.

The large voltage hysteresis observed (reaching almost 1V) in galvanostatic cycling profile of 1,2oximate derivatives (2-5) triggered our interest in further analyses of this aspect, as this could be in line with previous mentions on the electrochemical irreversibility in 1,4-dioxime derivatives ${ }^{26}$. Given that the simplest 1,2-oximate derivative (3) results in a liquid phase product upon oxidation, extensive physico-chemical characterization of the redox process reversibility was difficult to perform and therefore, lithiated diphenylglyoxime ( $\mathrm{Li}_{2}-\mathrm{DPGO}, 2$ ) was chosen as prototype to explore the solid-state electrochemical behavior of lithiated 1,2-oximates. On galvanostatic charging (oxidation) of Li 2 -DPGO, the charge potential reaches a flat plateau around $3.1 \mathrm{~V}$ with efficient extraction of two electrons, reaching the theoretical capacity (212 mAh g-1 , Fig. 3b, blue curve). The discharge, and subsequent cycling were found to be reversible, proceeding through a two-electron/lithium transfer, yet the discharge was observed at a much lower potential (in average, at about $2.1 \mathrm{~V} \mathrm{vs.} \mathrm{Li}^{+} / \mathrm{Li}$ ), giving rise to a polarization of nearly $1 \mathrm{~V}$. To confirm the reversibility of the chemical conversion process, the electrochemistry of the oxidized form of $\mathrm{Li}_{2}$-DPGO, namely the 3,4-diphenyl-1,2,5-oxadiazole-2-oxide (DPODO), was also tested. The galvanostatic charge-discharge profile of DPODO active electrode material (given the oxidized state of DPODO, the cell was started in discharge-reduction mode) displayed comparable electrochemical cycling profile (Fig. 3b, black curve), confirming the chemical and electrochemical reversibility between $\mathrm{Li}_{2}-\mathrm{DPGO}$ and DPODO phases.

The reversible redox of the $\mathrm{Li}_{2}$-DPGO $\leftrightarrow$ DPODO, as well as of other 1,2-oximate derivatives, conversion process consistently displayed high polarization $(\sim 0.6-1 \mathrm{~V})$, persistent during subsequent charge-discharge cycles. Galvanostatic intermittent titration technique (GITT) analysis shows that the large part of polarization ( $80 \%$ ) originates from the oxidation (charge) process (Fig. S28). As schematized in Fig. $2 b$, the open chain dioximate undergoes significant structural modification including oximate to nitroso oxidative conversion, $E-Z$ configuration change, and furoxan cyclization. The electrode polarization is the result of many factors and parameters associated with the cell design (e.g. - electrolyte conductivity, separator thickness, etc), electrode design (carbon content, mass loading, etc), as well as to potential intrinsic material redox kinetics. In organic compounds, polarization can also originate from molecular orbital rearrangements during redox, which may require additional energy, 
and thus result in high polarization or low Faradaic (energy) efficiency. In the 1,2-oximate case, assuming the $E-Z$ isomerization process as the limiting kinetic step in solid-phase, and thus main responsible for the large polarization of the cells observed, we designed and studied the dilithium 9,10-phenanthrene dioximate $\left(\mathrm{Li}_{2}-\mathrm{PADO}, 4\right)$ expected to bypass the $E-Z$ isomerization given the molecularly locked configuration (Fig. S29), and thus result in lower polarization.

However, high large hysteresis of $\sim 1 \mathrm{~V}$ was also observed for the $\mathrm{Li}_{2}$-PADO active material, indicating that the $E-Z$ isomerization is not the main contributor to the cell polarization (Fig. $3 \mathrm{c}$ ). The experimental results have thus been corroborated to calculations for the energy profile of the $\mathrm{Li}_{2}-\mathrm{DPGO} \leftrightarrow$ DPODO conversion process (analysing both, linear and closed structure reaction pathways, and in both, gas and solid phases). The analysis shows a dependence between structure type (linear or closed) and the oxidation state, and that the structural change comes with an energy barrier of around $1 \mathrm{eV}$ between reduced and oxidized states (Fig. 3e, and refer to SI for more DFT details). The significant orbital rearrangement requires expense of energy which is reflected in the form of redox hysteresis or polarization. The computational results also indirectly explain the absence of polarization in 1,4 -oximate (e.g., $\left.\mathrm{Li}_{2}-\mathrm{BQDO}\right)$ which is due to minimal or no structural changes during the redox process. Moreover, small energy barrier posed by availability of more than one coordination modes of Li-ions is escaped by the spontaneity of PNND polymer formation. The polymerization is also energetically favorable due to low enthalpy and high negative entropy of activation $\left(-179.7 \text { to }-183.0 \mathrm{~J} \mathrm{~K}^{-1} \mathrm{~mol}^{-1}\right)^{31}$.

Unlike carbonyl-based redox systems, the conjugated oximates family is not limited to two-electron redox chemistry. By adopting well-defined molecular engineering methodologies, multiple oximate units have been further incorporated onto a single hydrocarbon backbone and higher capacity attained. The alicyclic $\alpha$-oximate ( $\left.\mathrm{Li}_{4}-\mathrm{TMTO}(5)\right)$ with four oximate reaches a 4-electron redox, with a theoretical specific capacity of $310 \mathrm{mAh} / \mathrm{g}$. The use of $\mathrm{Li}_{4}-\mathrm{TMTO}$ as active material displayed a charge plateau at 3.5 $\mathrm{V}$ (vs. $\mathrm{Li}^{+} / \mathrm{Li}$ ) followed by a discharge process at an average potential of $2.65 \mathrm{~V}$ (vs. $\mathrm{Li}^{+} / \mathrm{Li}$, Fig. $3 \mathrm{~d}$ ). The average redox potential of $\mathrm{Li}_{4}-\mathrm{TMTO}\left(\sim 3.0 \mathrm{~V} \mathrm{vs}\right.$. $\left.\mathrm{Li}^{+} / \mathrm{Li}\right)$ is approximately $400 \mathrm{mV}$ higher than that of $\mathrm{Li}_{2-}$

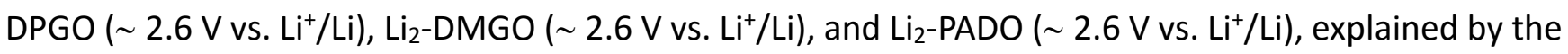
electron-withdrawing inductive effect $(-\mathrm{I})$ of the four $-\mathrm{OCH}_{3}$ groups. 


\section{Structural and chemical reversibility}

The reversible electrochemical polymerization - depolymerization of the $\mathrm{Li}_{2}-\mathrm{BQDO} \leftrightarrow \mathrm{PNND}$, or cyclization of the $\mathrm{Li}_{2}$-DPGO $\leftrightarrow$ DPODO processes, all accompanying the charge storage in solid phase, have been also monitored by in-operando XRD and ex-situ infrared spectroscopy (Fig. 4, and Fig. S30 S32) to confirm the structural and chemical reversibility, but also to attempt to further understand the dynamics of these processes in solid-phase. While the solvated $\mathrm{Li}_{2}-\mathrm{BQDO} \bullet 8 \mathrm{CH}_{3} \mathrm{OH}$ intermediated was crystalline, the desolvation process (necessary for the electrochemical tests) resulted in the formation of an amorphous $\mathrm{Li}_{2}-\mathrm{BQDO}$ phase (Figs. 4a, S31). Interesting, during the charge (oxidation) of $\mathrm{Li}_{2}-\mathrm{BQDO}$, the progressive appearance of the crystalline PNND phase was observed, which was characterized by the main diffraction peaks at $15.5^{\circ}$ and $25^{\circ}$, corresponding to (020) and (011) reflections plane respectively in the PNND phase (Fig. $4 \mathrm{a}$ and S31). These observations clearly corroborate the fact that the product of solid-phase electrochemical oxidation of $\mathrm{Li}_{2}-\mathrm{BQDO}$ is PNND, with the transient formation of 1,4-dinitrosobenzene, that undergoes polymerization as discussed earlier. It should be mentioned that the molecular dinitroso form exists in dissolved phase in low amounts (see earlier discussion on results of chemical analysis and solubility of PNND). However, the co-existence of this form in solid phase at room temperature remains highly unlikely, where trace amounts remain undetectable by available techniques, according to previous studies and current findings.

During the subsequent reduction-depolymerization process (i.e. discharge/lithiation), the XRD peaks (of PNND phase) progressively vanished, with complete amorphization of the active material observed at the end of this step. This process was found reversible during the second full cycle (Fig. 4a). Although the phase structure of the $\mathrm{Li}_{2}-\mathrm{BQDO}$ could not be determined from these data, nor that the same amorphous phase is generated upon electrochemical reduction (at the end of the two full galvanostatic cycles) we hypothesize that the $\mathrm{Li}_{2}-\mathrm{BQDO} \leftrightarrow$ PNND inter-conversion is a synchronous biphasic process with the structural reversibility of the PNND phase. Additional support to this is also provided by the flat-plateau charge-discharge galvanostatic profiles (Figs. 3 and S25), characteristic of bi-phasic reactions. It has to be however specified that this mechanistic view remains valid only when treating the equilibrium phases, and is therefore insufficient as it does not take into account the transient dinitroso intermediate, with direct investigation of the dynamic process being required for this. 


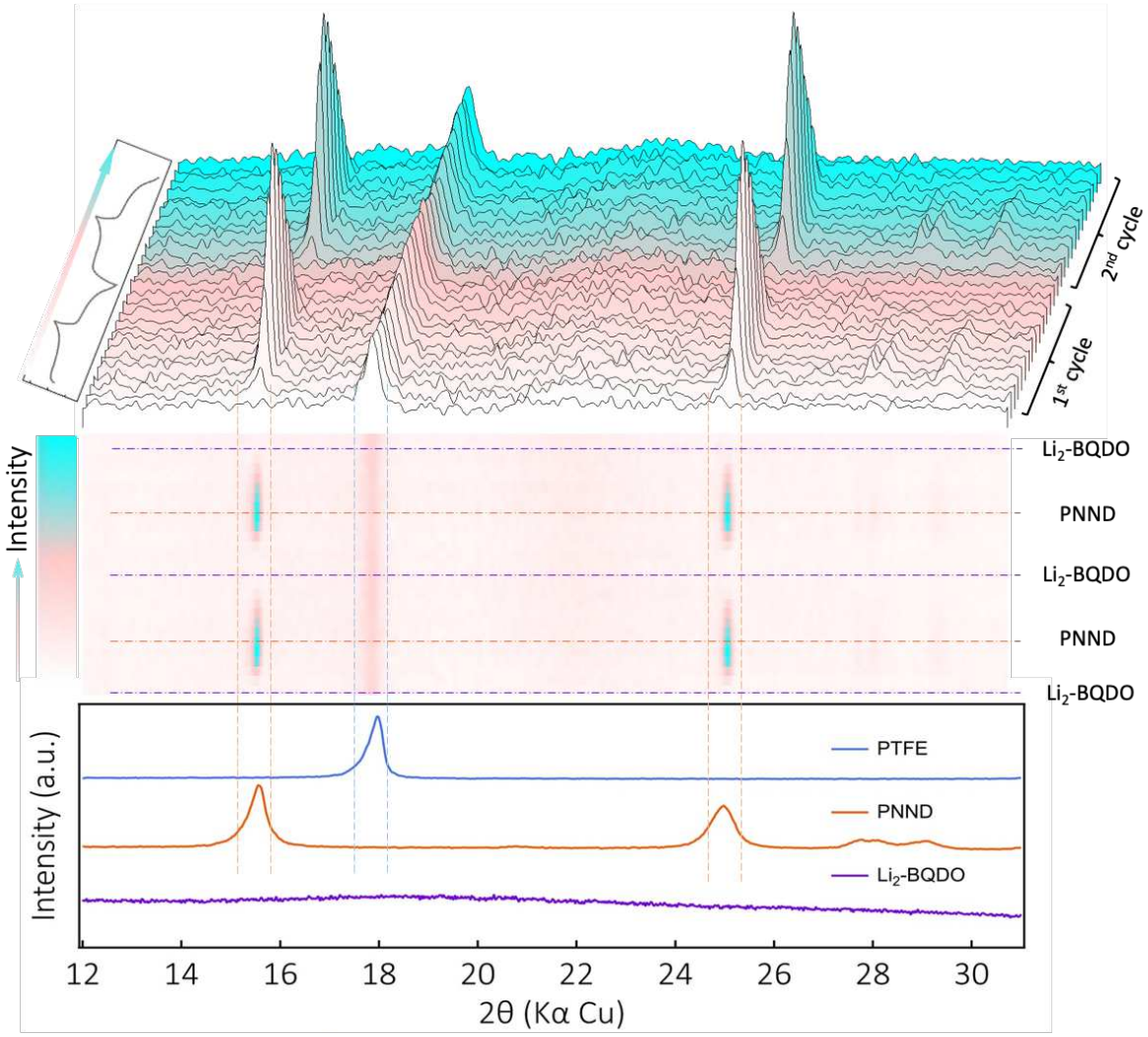

b

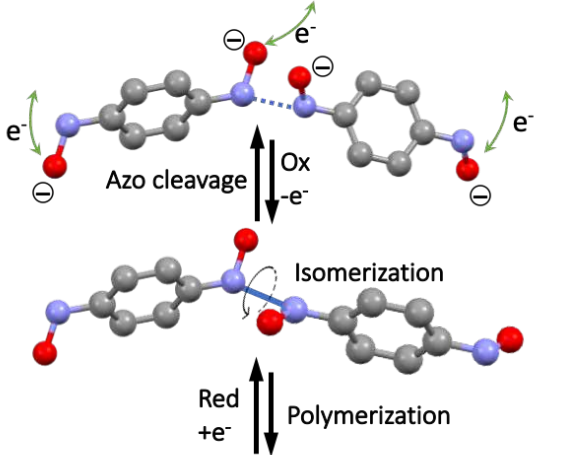

C
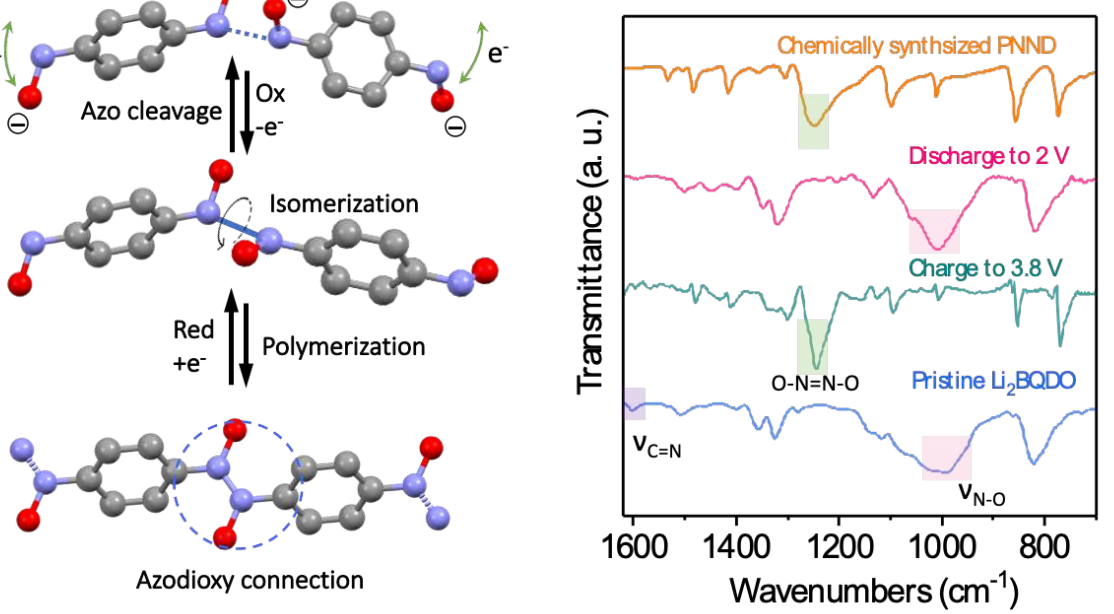

Figure 4. In-situ and ex-situ survey of the $\mathrm{Li}_{2}-B Q D O \leftrightarrow$ PNND conversion process in solid-phase. (a) Top panels: In-situ XRD of $\mathrm{Li}_{2}-B Q D O$ electrochemical conversion over the course of two full galvanostatic cycles. Bottom panel: overlayed PXRD patterns of PTFE binder used for the in-situ cell construct, as well as of pure, chemically synthesized $L_{2}-B Q D O$ and $P N N D$ phases. The $X$-axis $(2 \Theta, K \alpha-C u)$ is common to both top, and bottom panels. (b) Schematic illustration of the in-situ electrochemical polymerization-depolymerization process. (c) Ex-situ FTIR survey of the $L_{2}-B Q D O \leftrightarrow P N N D$ conversion process acquired at different charge states: pristine, fully charged to $3.8 \mathrm{~V}$ (corresponding to electrochemically generated PNND) and fully discharged to $2 \mathrm{~V}$ (corresponding to electrochemically generated $\left.L_{2}-B Q D O\right)$. For comparison, the FTIR pattern of chemically synthesized PNND is also displayed. 
Given the ambiguity of the phase reversibility upon redox, the chemical reversibility of the redoxdriven solid-phase polymerization was additionally confirmed by ex-situ FTIR analysis (Fig. 4c). On oxidation, the characteristic $\mathrm{V}_{\mathrm{N}-\mathrm{O}}$ and $\mathrm{V}_{\mathrm{C}=\mathrm{N}}$ bands of $\mathrm{Li}_{2}$-BQDO disappeared, accompanied by the appearance of E-azodioxy signature $\left(1264 \mathrm{~cm}^{-1}\right)$, identical to the chemically synthesized PNND phase. The characteristic FTIR band for Z-azodioxy conformer (1350-1400- $\mathrm{cm}^{-1}$ ) was not detected, hence this configuration can be excluded in the electrochemically generated PNND. Upon subsequent discharge, the FTIR spectrum was found to be closely similar to that of the pristine Li - BQDO (Fig. 4c) further confirming the reductive depolymerization to Li2-BQDO. On similar lines, in-situ XRD and ex-situ FTIR survey of the electrochemistry in solid-phase of $\alpha$ - or ortho-dioximates (e.g., Li $2_{2}$-DPGO $\leftrightarrow$ DPODO) also revealed the complete chemical and structural reversibility between the reduced (oximate form, $\mathrm{Li}_{2-}$ DPGO) and oxidized compounds (furoxan form, DPODO) (Fig. S30, S32). These findings not only unravel the standing issue of reversibility of the electrochemical conversion between oximates and azodioxy or furoxan forms but also give guidelines for future researchers to unambiguously design new oximate cathodes and redox pathway to fully achieve the potential of the materials.

Though it is rarely observed with organic cathode materials, this type of reversible structural transformation (polymerization - depolymerization) during solid-state electrochemical processing remains exclusive and highly efficient in oximate-based redox systems. Electrochemical polymerization of redox systems followed by their utilization as battery active electrode materials is a relatively wellknown process and has been largely applied with conducting polymers (PANI, PEDOT-PSS, and other). ${ }^{39,40}$ There are also more exotic examples, such as the in-situ electro-polymerization of $4,4^{\prime}, 4^{\prime \prime}$ Tris(carbazol-9-yl)-triphenylamine give the ability of carbazole groups to polymerize under an electric field and subsequently used as a $p$-type high-voltage redox-active charge storage material. ${ }^{41}$ There are nevertheless subtle yet fundamentally significant differences between the case of $\mathrm{Li}_{2}$-BQDO $\leftrightarrow$ PNND (disclosed here) and prior publications. The process presented in this work is a reversible biphasic electrochemical polymerization - depolymerization process, with synchronous $n$-type charge storage, whereas previous studies, rely on distinct two-step processing, consisting of initial irreversible electropolymerization, followed by the use of thus formed polymeric material for $p$-type charge storage. 


\section{Material level energy metrics evaluation and future perspectives}

a

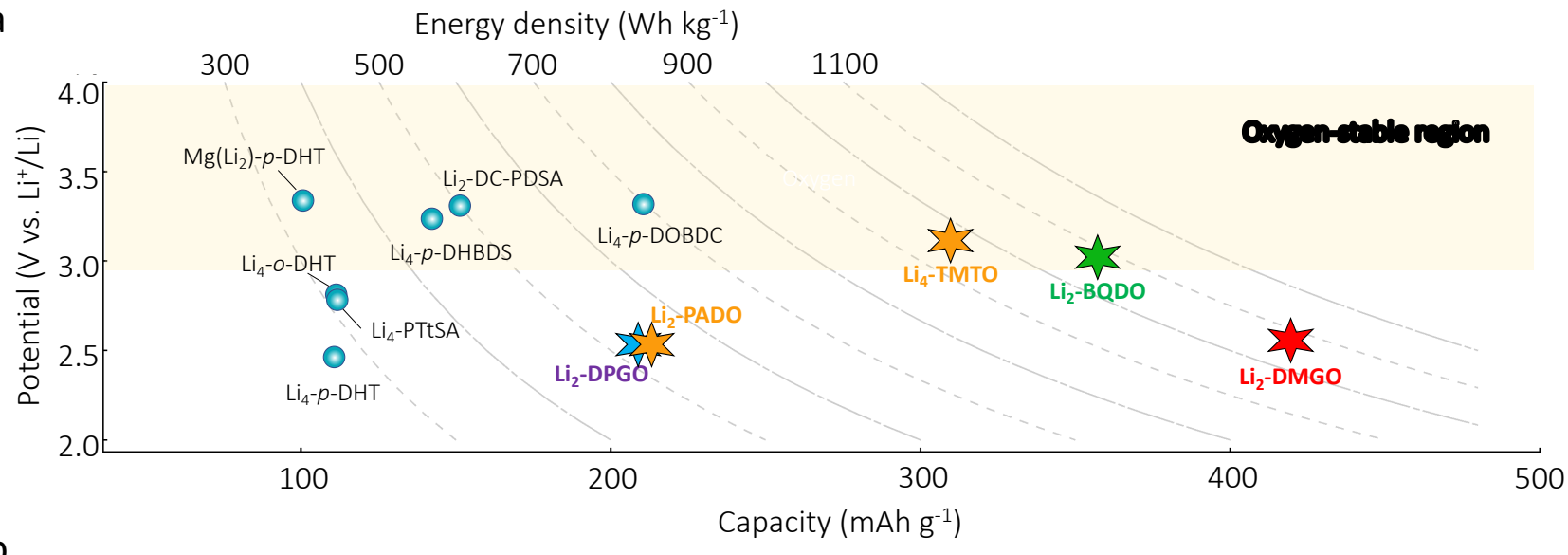

b
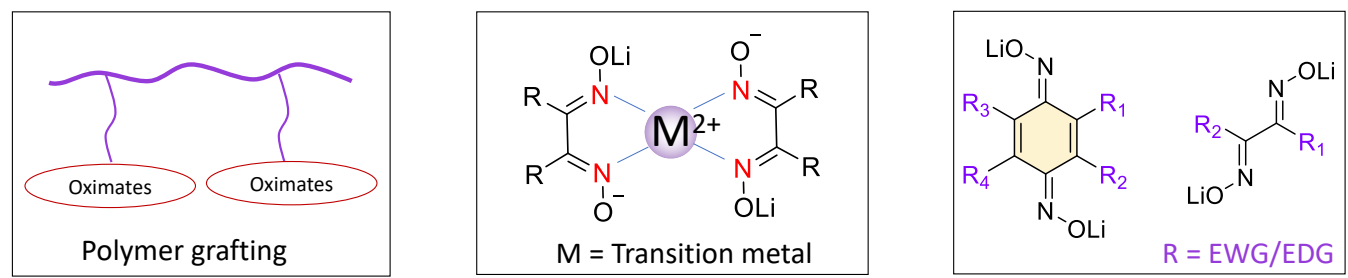

Figure 5. Material level energy metrics and future perspectives of oximate materials for energy storage. (a) Reversible capacity, average redox potential and the corresponding energy density of oximates (1-5, marked with a star symbol) as compared to state-of-art n-type organic positive electrode materials. (b) The inspiration to improve the cell practical performance can be drawn from the rich family of carbonyl battery electrodes, including polymer side-chain grafting, coordination chemistry approaches (coordination polymers, metalorganic frameworks), or functionalization with electron withdrawing-donating groups for redox potential tuning and solubility limitations.

Although cycling stability remains still of concern for majority of organic battery materials,

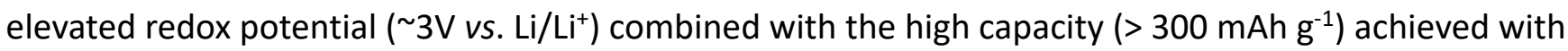
few oximate-based positive electrode materials are encouraging, considering the fundamental level of their technological development. The combination of high capacity and redox potential of this new class of organic positive electrode materials paves the realization of long-pending goal of verging the energy density range of $1 \mathrm{kWh} \mathrm{kg}^{-1}$ (energy metric established at the material level), establishing superiority over the existing conjugated enolates, conjugated sulfonamides and other organic battery material classes (in the range of 300-700 $\mathrm{Wh} \mathrm{kg}^{-1}$, Fig. 5a and Table S5). Although this value stands considerably 
higher than the energy content of many conventional inorganic positive electrode materials, it is also too early to consider the fundamental developments of this first generation of conjugated oximate battery electrode materials for commercialization, demanding further improvement of the overall performance through various molecular architecture design, that could be inspired from the rich knowledge of quinone analogues (Fig. 5b). ${ }^{42,43}$ For example, oximate redox centers can be incorporated within polymeric backbone wherein the distinct redox features can still be achieved with improved cycling stability. The $\alpha$-dioximates are also known to act as chelating groups to transition metal ions forming coordination complexes or metal-organic frameworks. ${ }^{44}$ This could be also exploited and could potentially lead to redox potential increase through cation inductive effect, and capacity increase if the redox of the metal ion can be accessed ${ }^{12,17}$. The conjugated oximates also offer the possibility of redox potential tuning by functionalizing with electron withdrawing or donating groups (e.g., methoxy, carboxylate or sulfonate - as for instance discussed for $\mathrm{Li}_{4}-\mathrm{TMTO}$ ) and the chemical space of conjugated oximate electrode materials is by no means limited to the five examples investigated in this work. Since oxime function can be easily accessed by the reaction of hydroxylamine with carbonyl derivatives, it is not excessive to assume that many of the quinone derivatives reported so far charge storage can be adapted to oximates, thereby leading to a library of new organic Li-ion positive electrode materials.

\section{Summary}

The reversible redox of conjugated oximates in solid-phase is revealed and shown to fulfill the requirements for performant Li-ion storage. When compared to carbonyl based redox systems, unique molecular design and redox mechanism of the oximate groups offers net advantages in terms of dry air stability, increased energy content, as well as higher structural versatility allowing the implementation of both cyclic and acyclic derivatives as electrode materials. All studied chemistries undergo synchronous one-electron redox per oximate unit to form nitroso derivatives, followed by significant yet reversible structural modifications, including polymerization-depolymerization for cyclic 1,4dioximates (e.g., Li 2 -BQDO, 1) or intermolecular furoxan cyclization for alicyclic 1,2-dioximates (2 - 4). According to design rationales (Fig. 1), the oximate chemistries display high redox potential (2.5-3.2 V vs. $\mathrm{Li}^{+} / \mathrm{Li}$ ) and are stable towards oxidation in dry air, important aspects to consider for practical Li-ion positive electrode materials. The low molecular mass results in high specific capacities attained (in the range of 210-360 $\mathrm{mAh} \mathrm{g}^{-1}$ ), which translates energy content at par with current technologies and 
materials. Creeping towards practical organic batteries, the versatility of organic chemistry for further molecular engineering and design of oximate family provides umpteen opportunities to further upgrade the cell parameters such as redox potential, capacity and cycling stability.

\section{Acknowledgments}

J.W., X.L., M.D., Y.Z., and X.G acknowledge China-Belgium (Wallonia-Brussels French-speaking Region) Outstanding Young Scholar Scholarships for the financial support. A.V. is indebted to the European Research Council under the European Union's Horizon 2020 research and innovation program (grant agreement no. 770870, MOOiRE), as well as partial support from F.R.S.-FNRS through F.4552.21P and J.0168.22 grants. C.M. acknowledge UEFISCDI for financial support, grant PN-III-P4-ID-PCE-20200824, Contract nr. PCE-22/2021. The authors declare no competing financial interests.

\section{Data Availability Statement}

The data that support the findings of this study are available from the corresponding author upon reasonable request.

\section{References}

1 Poizot, P. et al. Opportunities and Challenges for Organic Electrodes in Electrochemical Energy Storage. Chem Rev 120, 6490-6557, doi:10.1021/acs.chemrev.9b00482 (2020).

2 Esser, B. et al. A perspective on organic electrode materials and technologies for next generation batteries. Journal of Power Sources 482, doi:10.1016/j.jpowsour.2020.228814 (2021).

3 Lakraychi, A. E., Dolhem, F., Vlad, A. \& Becuwe, M. Organic Negative Electrode Materials for Metal-lon and Molecular-Ion Batteries: Progress and Challenges from a Molecular Engineering Perspective. Advanced Energy Materials 11, doi:10.1002/aenm.202101562 (2021).

$4 \quad$ Wang, J. \& Vlad, A. Empowering magnesium. Nature Energy 5, 945-946, doi:10.1038/s41560-020-00744-y (2020).

5 Schon, T. B., McAllister, B. T., Li, P. F. \& Seferos, D. S. The rise of organic electrode materials for energy storage. Chem Soc Rev 45, 6345-6404, doi:10.1039/c6cs00173d (2016).

6 Gottis, S., Barres, A. L., Dolhem, F. \& Poizot, P. Voltage gain in lithiated enolate-based organic cathode materials by isomeric effect. ACS App/ Mater Interfaces 6, 10870-10876, doi:10.1021/am405470p (2014).

7 Jia, H. et al. Core-shell nanostructured organic redox polymer cathodes with superior performance. Nano Energy 64, doi:10.1016/j.nanoen.2019.103949 (2019).

8 Jouhara, A. et al. Tuning the Chemistry of Organonitrogen Compounds for Promoting All-Organic Anionic Rechargeable Batteries. Angew Chem Int Ed Eng/58, 15680-15684, doi:10.1002/anie.201908475 (2019).

9 Dominique, G. J.-M., Tarascon. Rocking-chair or lithium-ion rechargeable lithium batteries. Advanced Materials 6, 408-212, doi:org/10.1002/adma.19940060516 (1994). 
Sid, M. B., Scrosati. Lithium-ion rechargeable batteries. Journal of Power Sources 51, 79-84, doi:org/10.1016/0378-7753(94)01956-8 (1994).

11 Lakraychi, A. E. et al. An air-stable lithiated cathode material based on a 1,4-benzenedisulfonate backbone for organic Li-ion batteries. Journal of Materials Chemistry A 6, 19182-19189, doi:10.1039/c8ta07097k (2018).

12 Jouhara, A. et al. Raising the redox potential in carboxyphenolate-based positive organic materials via cation substitution. Nat Commun 9, 4401, doi:10.1038/s41467-018-06708-x (2018).

13 Rambabu, D. et al. An Electrically Conducting Li-Ion Metal-Organic Framework. J Am Chem Soc 143, 1164111650, doi:10.1021/jacs.1c04591 (2021).

14 Sieuw, L. et al. Through-Space Charge Modulation Overriding Substituent Effect: Rise of the Redox Potential at $3.35 \mathrm{~V}$ in a Lithium-Phenolate Stereoelectronic Isomer. Chemistry of Materials 32, 9996-10006, doi:10.1021/acs.chemmater.0c02989 (2020).

15 Wang, J. et al. Conjugated sulfonamides as a class of organic lithium-ion positive electrodes. Nat Mater $\mathbf{2 0}$, 665-673, doi:10.1038/s41563-020-00869-1 (2021).

16 Wang, J. et al. A High-Voltage Organic Framework for High-Performance Na- and K-lon Batteries. ACS Energy Letters 7, 668-674, doi:10.1021/acsenergylett.1c02571 (2022).

17 Wang, J. et al. High performance $\mathrm{Li}-, \mathrm{Na}-$, and $\mathrm{K}$-ion storage in electrically conducting coordination polymers. Energy \& Environmental Science 15, 3923-3932, doi:10.1039/d2ee00566b (2022).

18 Yang, $\mathrm{H}$. et al. Molecular engineering of carbonyl organic electrodes for rechargeable metal-ion batteries: fundamentals, recent advances, and challenges. Energy \& Environmental Science 14, 4228-4267, doi:10.1039/d1ee00419k (2021).

19 Armand, M. et al. Conjugated dicarboxylate anodes for Li-ion batteries. Nat Mater 8, 120-125, doi:10.1038/nmat2372 (2009).

20 Song, Z. et al. Polyanthraquinone as a Reliable Organic Electrode for Stable and Fast Lithium Storage. Angew Chem Int Ed Eng/54, 13947-13951, doi:10.1002/anie.201506673 (2015).

21 Chen, $\mathrm{H}$. et al. From biomass to a renewable LixC6O6 organic electrode for sustainable Li-ion batteries. ChemSusChem 1, 348-355, doi:10.1002/cssc.200700161 (2008).

22 Guo, W., Yin, Y.-X., Xin, S., Guo, Y.-G. \& Wan, L.-J. Superior radical polymer cathode material with a twoelectron process redox reaction promoted by graphene. Energy Environ. Sci. 5, 5221-5225, doi:10.1039/c1ee02148f (2012).

23 Janoschka, T., Hager, M. D. \& Schubert, U. S. Powering up the future: radical polymers for battery applications. Adv Mater 24, 6397-6409, doi:10.1002/adma.201203119 (2012).

24 Beaudoin, D., Maris, T. \& Wuest, J. D. Constructing monocrystalline covalent organic networks by polymerization. Nat Chem 5, 830-834, doi:10.1038/nchem.1730 (2013).

25 Bibulić, P., Rončević, I., Bermanec, V. \& Vančik, H. Polymerization of 1,4-dinitrosobenzene: Kinetics and Submicrocrystal Structure. Croatica Chemica Acta 90, doi:10.5562/cca3220 (2017).

26 Rathore, R. J. S. K. J. K. K. Catalytic autoxidation of benzoquinone dioximes with nitrogen oxides: steric effects on the preparation of monomeric dinitrosobenzenes. Journal of the Chemical Society, Perkin Transactions 1 19, 2675-2684, doi:org/10.1039/P19940002675 (1994).

27 Kokkinidis, G. G. P. A reaction model for the ECECE mechanism: Reduction of p-benzoquinone dioxime on $\mathrm{Pt} / \mathrm{M}$ (upd) modified electrodes in $\mathrm{HClO}_{4}$ solutions. Journal of Electroanalytical Chemistry and Interfacial Electrochemistry 257, 239-255, doi:org/10.1016/0022-0728(88)87045-1 (1988).

28 Feuer, H. The Chemistry of the Nitro and Nitroso Groups, Part 1. (Interscience, 1969).

29 Gowenlock, B. G. \& Richter-Addo, G. B. Dinitroso and polynitroso compounds. Chem Soc Rev 34, 797-809, doi:10.1039/b500855g (2005).

30 Joseph H. Trepagnier, W., Del., and John V. Vaughen, Woodstown, N. J. Preparation of dinitrosobenzenes (U.S. Patent No. 2,419,976). United States of American patent (1947).

31 Gallo, G., Mihanović, A., Rončević, I., Dinnebier, R. \& Vančik, H. Crystal structure and ON-OFF polymerization mechanism of poly(1,4-phenyleneazine-N,N-dioxide), a possible wide bandgap semiconductor. Polymer 214, doi:10.1016/j.polymer.2020.123235 (2021). 
32 Hacker, N. P. Investigation of the polymerization of 1,4-dinitrosobenzene by low-temperature infrared and UV absorption spectroscopy. Macromolecules 26, 5937-5942, doi:org/10.1021/ma00074a015 (1993).

33 Lorraine, A. M. C. B. G. G. I. J. M. Properties and spectroscopic studies of polymeric dinitrosobenzenes. Journal of the Chemical Society, Perkin Transactions 22, 243-245 (1992).

34 Sieuw, L. et al. A H-bond stabilized quinone electrode material for Li-organic batteries: the strength of weak bonds. Chem Sci 10, 418-426, doi:10.1039/c8sc02995d (2019).

35 Sillitoe, A. K. M. M. H. 3,4-Diphenylfurazan N-oxide. Acta Crystallographica Section B: Structural Crystallography and Crystal Chemistry 34, 2021-2023 (1978).

36 Yu, Z.-X. P. C. K. N. H. Dimerizations of Nitrile Oxides to Furoxans Are Stepwise via Dinitrosoalkene Diradicals:

A Density Functional Theory Study. Journal of the American Chemical Society 125, 15420-15425, doi:doi.org/10.1021/ja037325a (2003).

37 Zhao, Q., Zhu, Z. \& Chen, J. Molecular Engineering with Organic Carbonyl Electrode Materials for Advanced Stationary and Redox Flow Rechargeable Batteries. Adv Mater 29, doi:10.1002/adma.201607007 (2017).

38 Huskinson, B. et al. A metal-free organic-inorganic aqueous flow battery. Nature 505, 195-198, doi:10.1038/nature12909 (2014).

39 Sieuw, L., Ernould, B., Gohy, J. F. \& Vlad, A. On the improved electrochemistry of hybrid conducting-redox polymer electrodes. Sci Rep 7, 4847, doi:10.1038/s41598-017-05063-z (2017).

$40 \mathrm{Vlad}, \mathrm{A}$. et al. Exploring the potential of polymer battery cathodes with electrically conductive molecular backbone. Journal of Materials Chemistry A 3, 11189-11193, doi:10.1039/c5ta01500f (2015).

41 Zhao, C. et al. In Situ Electropolymerization Enables Ultrafast Long Cycle Life and High-Voltage Organic Cathodes for Lithium Batteries. Angew Chem Int Ed Eng/ 59, 11992-11998, doi:10.1002/anie.202000566 (2020).

42 Häupler, B., Wild, A. \& Schubert, U. S. Carbonyls: Powerful Organic Materials for Secondary Batteries. Advanced Energy Materials 5, doi:10.1002/aenm.201402034 (2015).

43 Shi, Y. et al. Quinone-Amine Polymer Nanoparticles Prepared through Facile Precipitation Polymerization as Ultrafast and Ultralong Cycle Life Cathode Materials for Lithium-Ion Batteries. Advanced Functional Materials 32, doi:10.1002/adfm.202111307 (2022).

44 Xie, L. S. et al. Isoreticular Linker Substitution in Conductive Metal-Organic Frameworks with Through-Space Transport Pathways. Angew Chem Int Ed Eng/59, 19623-19626, doi:10.1002/anie.202004697 (2020). 


\section{Supplementary information for}

Revealing the Reversible Solid-State Electrochemistry of Lithium-Containing Conjugated Oximates: Towards a New Functionality for Organic Batteries

Jiande Wang ${ }^{1, \S}$, Petru Apostol, ${ }^{1, \S}$, Darsi Rambabu ${ }^{1, \S}$, Xiaolong Guo ${ }^{1, \S}$, Xuelian Liu ${ }^{1}$, Koen Robeyns ${ }^{1}$, Mengyuan Du ${ }^{1}$, Yan Zhang ${ }^{1,2}$, Shubhadeep Pal ${ }^{1}$, Robert Markowski ${ }^{1}$, Fabio Lucaccioni ${ }^{1}$, Alae Eddine Lakraychi ${ }^{1}$, Cristian Morari ${ }^{3}$, Jean-François Gohy ${ }^{1}$, Deepak Gupta ${ }^{1 *}$ and Alexandru Vlad ${ }^{*}$

1 Institute of Condensed Matter and Nanosciences, Molecular Chemistry, Materials and Catalysis, Université catholique de Louvain, Louvain-Ia-Neuve B-1348, Belgium

${ }^{2}$ College of Materials Science and Engineering, Hunan Province Key Laboratory for Advanced Carbon Materials and Applied Technology, Hunan University, Changsha 410082, Hunan, P. R. China

${ }^{3}$ Institutul Național de Cercetare-Dezvoltare pentru Tehnologii Izotopice și Moleculare ClujNapoca, Cluj-Napoca, România

$\S$ These authors contributed equally to this work;

Corresponding authors: deepak.gupta@uclouvain.be, alexandru.vlad@uclouvain.be 


\section{Table of Contents}

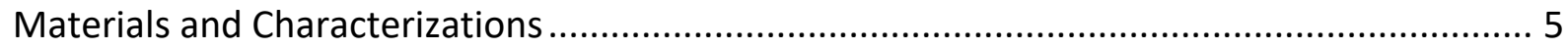

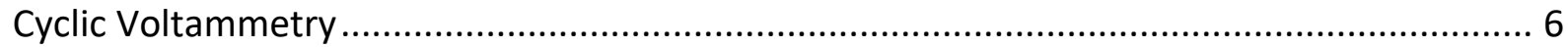

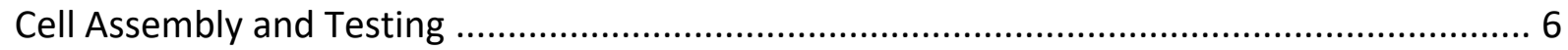

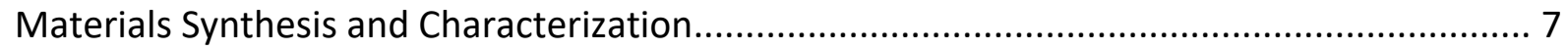

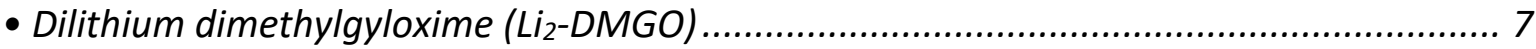

Supplementary Figure $S 1 .{ }^{1} \mathrm{H} N \mathrm{MR}(\mathrm{MeOD})$ spectrum of $\mathrm{Li}_{2}$-DMGO .............................. 7

Supplementary Figure S2. ${ }^{13} \mathrm{C}$ NMR (MeOD) spectrum of $\mathrm{Li}_{2}$-DMGO.............................. 8

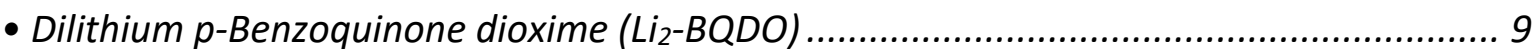

Supplementary Figure S3. ${ }^{1} \mathrm{H} N \mathrm{NMR}(\mathrm{MeOD})$ spectrum of $L i_{2}-B Q D O$.................................. 9

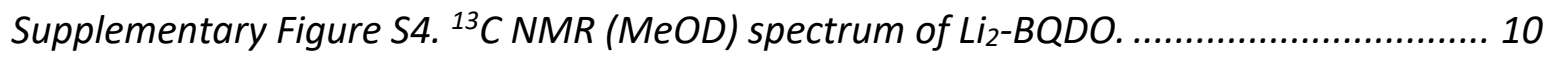

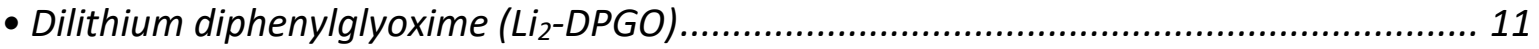

Supplementary Figure S5. ${ }^{1} \mathrm{H}$ NMR (MeOD) spectrum of $L i_{2}$-DPGO .............................. 11

Supplementary Figure S6. ${ }^{13} \mathrm{C}$ NMR (MeOD) spectrum of $\mathrm{Li}_{2}$-DPGO.............................. 12

-Dilithium 9,10-phenanthrenedione oximate (Li 2 -PADO) ............................................. 13

Supplementary Figure S7. ${ }^{1} \mathrm{H}$ NMR (DMSO-d6) spectrum of $\mathrm{H}_{2}-\mathrm{PADO}$............................... 13

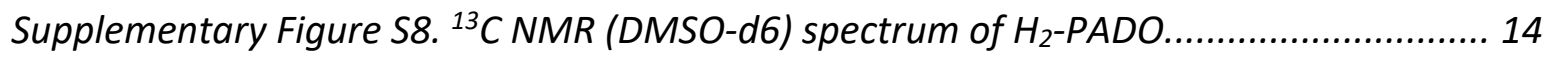

Supplementary Figure S9. ${ }^{1} \mathrm{H}$ NMR (MeOD) spectrum of $\mathrm{Li}_{2}$-PADO ................................. 15

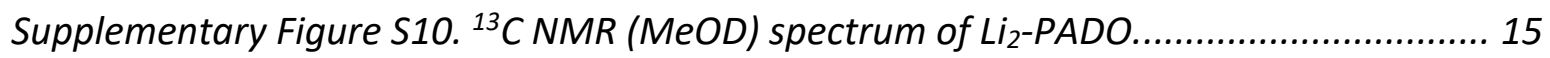

- Tetralithium-1,1,4,4-Tetramethoxy-2,3,5,6-tetraoximatecyclohexane (Li 4 -TMTO). ${ }^{4}$...... 16

Supplementary Figure S11. ${ }^{1} \mathrm{H}$ NMR and ${ }^{13} \mathrm{C} N M R(D M S O-d 6)$ spectra of $\mathrm{H}_{4}$-TMTO........... 16

Supplementary Figure S12. ${ }^{1} \mathrm{H}$ NMR (MeOD) spectrum of $\mathrm{Li}_{4}$-TMTO............................... 17

Supplementary Figure S13. ${ }^{13} \mathrm{C}$ NMR (MeOD) spectrum of $\mathrm{Li}_{4}$-TMTO .............................. 18

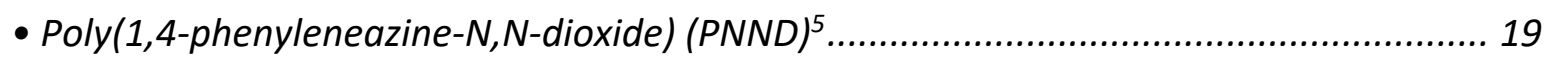

Crystal structure determination by X-ray powder diffraction method................................ 20

Supplementary Table S1. Powder XRD data and refinement parameters of PNND ${ }^{a} \ldots \ldots . . . .20$

Supplementary Table S2. Fractional Atomic Coordinates $(\times 104)$ and Equivalent Isotropic Displacement Parameters (Å2×103) for PNND ............................................................ 21

Supplementary Table S3. Bond Lengths for PNND..................................................... 21

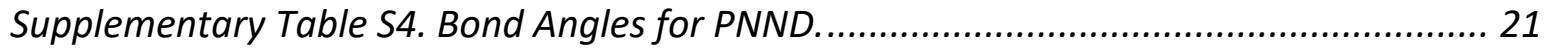

Supplementary Table S5. Reaction mechanisms and groups for electrochemical storage in reported redox-active organic materials 
Supplementary Figure S14. FTIR analysis survey for the synthesis of $L i_{2}-B Q D O$, coupled to air and moisture stability analysis.

Supplementary Figure S15. FTIR analysis survey for the synthesis of Li $i_{2}-D P G O, \ldots \ldots \ldots \ldots . . . . . .24$

Supplementary Figure S16. FTIR analysis survey for the synthesis of $L_{2}-D M G O, \ldots . . . . . . . . .25$

Supplementary Figure S17. FTIR analysis survey for the synthesis of $L_{4}-T M T O, \ldots \ldots \ldots \ldots . . . .26$

Supplementary Figure S18. FTIR analysis survey for the synthesis of $L i_{2}-P A D O, \ldots \ldots . . . . . . . . .27$

Supplementary Figure S19. Crystal structure analysis of PNND ..................................... 28

Supplementary Figure S20. Crystal structure of PNND polymer. .................................... 29

Supplementary Table S6. Solubility of electrode materials in 1M LiTFSI in EC/DMC electrolyte formulation. 30

Supplementary Figure S21. UV-visible solubility calibration plots of the studied materials and their unknown diluted solutions in 1M Li-TFSI in EC/DMC electrolyte. Inset figure in each figure is respective UV-visible spectra.. 31

Supplementary Figure S22. Rate capability test of $\mathrm{Li}_{2}-\mathrm{BQDO}$.

Supplementary Figure S23. The capacity retention and coulombic efficiency of $L_{2}-B Q D O$ cycled in a saturated LiTFSI in EC/DMC (1:1 vol\%) electrolyte. The cell was cycled a rate of $\mathrm{C} / 2$ (one electron exchange in $1 \mathrm{~h}$ ).

Supplementary Figure S24. Control experiment for the determination of the specific capacity contribution from Ketjen Black conductive carbon. .... 35

Supplementary Figure S25. Capacity-potential profile of chemically synthesized PNND and comparisons of cycling profiles of in different electrolytes. 36

Supplementary Figure S26. Comparison of electrochemical properties for the main building units studied in this work: dilithium-1,4-benzenediolate $\left(L i_{2}-B Q\right)$ vs. dilithium $p$ -

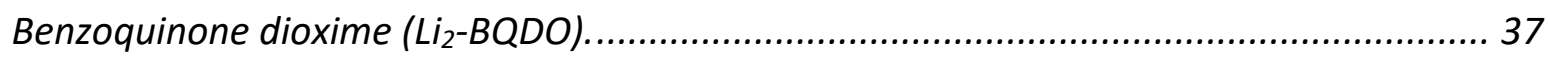

Supplementary Figure S27. Solid-state electrochemistry of dilithium-dimethylgyloxime ( $\mathrm{Li}_{2^{-}}$ DMGO).

Supplementary Figure S28. Galvanostatic Intermittent Titration Technique (GITT) plot of the $L i_{2}-D P G O-D P O D O$ redox as measured in a Li half cell configuration.

Supplementary Figure S29. Structural reorganization of $L i_{2}-D P G O$ and $L i_{2}-P A D O$ during the oxidation process.

Supplementary Figure S30. Ex-situ FTIR survey of reversible electrochemical reaction between $L_{2}-D P G O$ and DPODO in solid phase.

Supplementary Figure S31. PXRD data of $L i_{2}-B Q D O$ and PNND phases and analysis of $L_{2_{2}-}$ $B Q D O-8 M e O H$ crystal structure.

Supplementary Table S7. Crystal data and structure refinement for Li2-BQDO-8MeOH.... 43 Supplementary Figure S32. In-situ XRD survey of $L i_{2}-D P G O$ during electrochemical cycling in solid phase. 
Supplementary Figure S33. SEM images of pristine PNND and $\mathrm{Li}_{2} \mathrm{BQDO}$ at different cycling

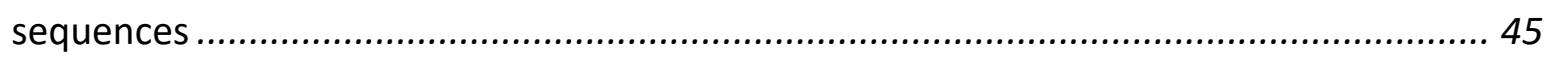

Gel Permeation Chromatography (GPC) analysis of soluble PNND species........................ 46

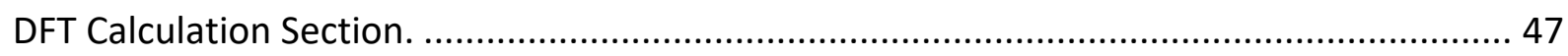

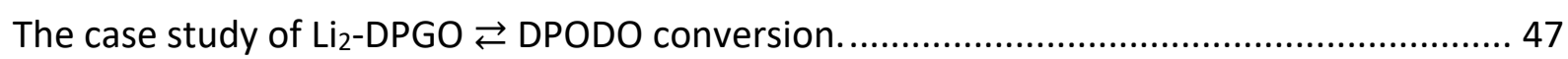

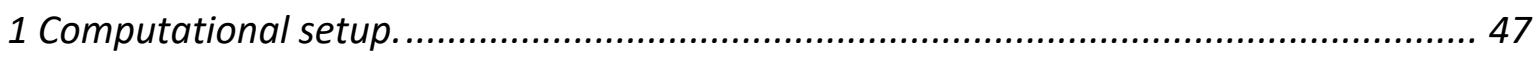

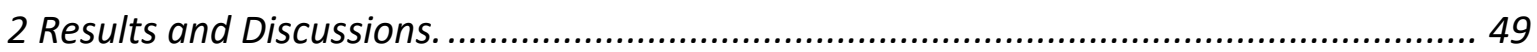

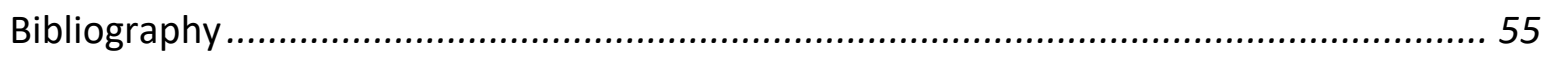




\section{Materials and Characterizations}

1,4-benzoquinone dioxime (95\%, TCl Chemicals), Dimethylglyoxime (98\%, $\mathrm{TCl}$ Chemicals), anti-Diphenylglyoxime (97\%, Sigma Aldrich) and 3,4-diphenyl-1,2,5-oxadiazole-2-oxide (DPODO, Sigma Aldrich) were used as received. Lithium bis(trifluoromethylsulfonyl)imide (LiTFSI), Ethylene Carbonate (EC), Dimethyl Carbonate (DMC) and Tetragylyme were obtained from Dodo Chem, China. Anhydrous solvents (THF, DMF, MeOH and Diethyl ether) for synthesis were purchased from Across Organics and used as received.

Fourier transform Infra-Red (FTIR) spectroscopy was carried out on Agilent Technologies Cary 630 FTIR operated in transmission or ATR mode. Nuclear Magnetic Resonance (NMR) spectra were recorded with a Bruker magnet system $300 \mathrm{MHz} / 54 \mathrm{~mm}$ ultrashield spectrometer. Dimethyl sulfoxide-d6 was used as solvent and internal standard for chemical shifts. X-ray powder diffraction (XRD) patterns were collected on STOE DARMSTADT Transmission diffractometer system using $\mathrm{Cu} / \mathrm{Mo} \mathrm{K} \alpha 1$ radiation with a wavelength of 1.540600/0.70930 Å. UV-visible spectroscopy studies were carried out using a Shimadzu UV1700 PharmaSpec.

SEM images were recorded on a JEOL FEG SEM 7600F (JEOL, Tokyo, Japan) Zeiss Neon 40 cross-beam workstation with Gemini SEM column (Carl Zeiss Iberia, S.L, Madrid, Spain).

HRMS was measured using Thermo Scientific ${ }^{\mathrm{TM}} \mathrm{Q}$-Exactive Orbitrap mass spectrometer and $\mathrm{APCl}+$ as ionisation mode.

The GPC was performed on an Agilent gel permeation chromatography (GPC) system equipped with an Agilent $1100 / 1200$ pump ( $25^{\circ} \mathrm{C}$; eluent: DMF, $2.5 \mathrm{mM}$ of $\mathrm{NH}_{4} \mathrm{PF}_{6}$; flow rate: $1 \mathrm{~mL} / \mathrm{min})$.

Elemental analysis (CHNS) was done with Thermo Scientific ${ }^{\mathrm{TM}}$ FlashSmart $^{\mathrm{TM}}$ Elemental Analyzer.

X-ray crystallography: Single crystal X-ray data collection on $\mathrm{Li}_{2}-\mathrm{BQDO}-8 \mathrm{MeOH}$ was carried out on a Xcalibur, Ruby, Gemini ultra-instrument using Cu-K $\alpha$ radiation (1.54184 ̊̊) generated by a fine-focus sealed X-Ray tube. Prior to measurement, the crystal was flash cooled to 100 $\mathrm{K}$ in an $\mathrm{N}_{2}$ gaseous stream. Data acquisition, reduction and analytical face-index based absorption correction were made using the program CrysAlis $^{1}$ and the implemented absorption correction was applied. The structure was solved by SHELXT and refined by SHELXL2014/7. ${ }^{2}$ Anisotropic displacement parameters were applied for all atoms, except hydrogen atoms. All non-hydrogen atoms were refined anisotropically and $\mathrm{H}$ atoms were placed on calculated positions and refined in riding mode on their parent atoms with isotropic temperature factors, fixed at $1.2 \mathrm{Ueq}$ of the parent atoms. CCDC number 2193545 (for $\mathrm{Li}_{2}$ BQDO-8MeOH) contains the supplementary crystallographic data for this paper. These data can be obtained free of charge from The Cambridge Crystallographic Data Centre via www.ccdc.cam.ac.uk/structures 


\section{Cyclic Voltammetry}

Cyclic Voltammetry was conducted with a BioLogic Science Instruments SP-300. A threeelectrode setup with screen-printed platinum as working and counter electrode and screenprinted $\mathrm{Ag}$ as the pseudo-reference electrode was used for measurements. A solution of $0.1 \mathrm{M}$ $\mathrm{LiCl}$ in DMSO was used as supporting electrolyte. Typically, $2 \mathrm{mM}$ of the active material was dissolved in the electrolyte solution, and the CV was measured at a scan rate of $100 \mathrm{mV} \mathrm{s}^{-1}$. After the respective scan, $2 \mathrm{mM}$ of ferrocene was added to the solution and used as an internal reference for redox potential determination.

\section{Cell Assembly and Testing}

The electrodes were prepared by hand mixing and grinding the active material (50\% wt.), conductive carbon (40\% wt.) and PTFE binder (10\% wt.) for $15 \mathrm{~min}$. The formed powder mixture was added onto the positive side of a coin cell case and pressed with a stainless-steel disk. A typical mass loading of $4 \mathrm{mg} / \mathrm{cm}^{2}$ was used. The electrochemical properties and galvanostatic charge/discharge tests were performed in 2032-type coin cells with $13 \mathrm{~mm}$ lithium chips as the counter and pseudo-reference electrodes, one sheet of glass microfiber filter (Whatman GF/D, Aldrich) as separator, with different electrolyte formulations: 5 M LiTFSI in tetraglyme and $7 \mathrm{M}$ LiTFSI in EC/DMC. Galvanostatic charge/discharge tests were performed on Neware battery testing system at ambient temperature. GITT was tested under a galvanostatic current pulse of $\mathrm{C} / 10$ for a duration of $2 \mathrm{~h}$ followed by relaxation at open circuit for $1 \mathrm{~h}$ at each step. 


\section{Materials Synthesis and Characterization}

\section{- Dilithium dimethylgyloxime ( $\mathrm{Li}_{2}$-DMGO)}

Dimethylgyloxime $(1.16 \mathrm{~g}, 10 \mathrm{mmol})$ was dissolved in anhydrous methanol $(20 \mathrm{ml})$ and Lithium methoxide $(760 \mathrm{mg}, 20 \mathrm{mmol})$ was added to the solution under stirring. The reaction (performed in an Argon filled glove box), was allowed to stir for $24 \mathrm{~h}$ at room temperature. The yellow reaction mixture was poured into $100 \mathrm{~mL}$ of anhydrous diethyl ether to precipitate the product, followed by filtration, washing with copious amounts of diethyl ether and drying at $60^{\circ} \mathrm{C}$ under vacuum. The product ( $\left.\mathrm{Li}_{2}-\mathrm{DMGO}\right)$ was obtained in quantitative yield.

- ${ }^{1} \mathrm{H}$ NMR (300 MHz, MeOD) $\delta 2.01$ ppm. ${ }^{13} \mathrm{C}$ NMR (75 MHz, MeOD) $\delta 154.95,8.38$ ppm.

- HRMS (ESI) calcd for $\mathrm{C}_{4} \mathrm{H}_{6} \mathrm{Li}_{2} \mathrm{~N}_{2} \mathrm{O}_{2}: 128.0749$, Found: $127.9790[\mathrm{M}]^{+}$.

- Elemental Analysis: Calculated: C: 37.54\%; H: 4.73\%; N: 21.89\%. Found: C: 37.38\%; H: 5.03\%; $\mathrm{N}: 20.10 \%$.

Supplementary Figure S1. ${ }^{1} \mathrm{H}$ NMR (MeOD) spectrum of $\mathrm{Li}_{2}$-DMGO.

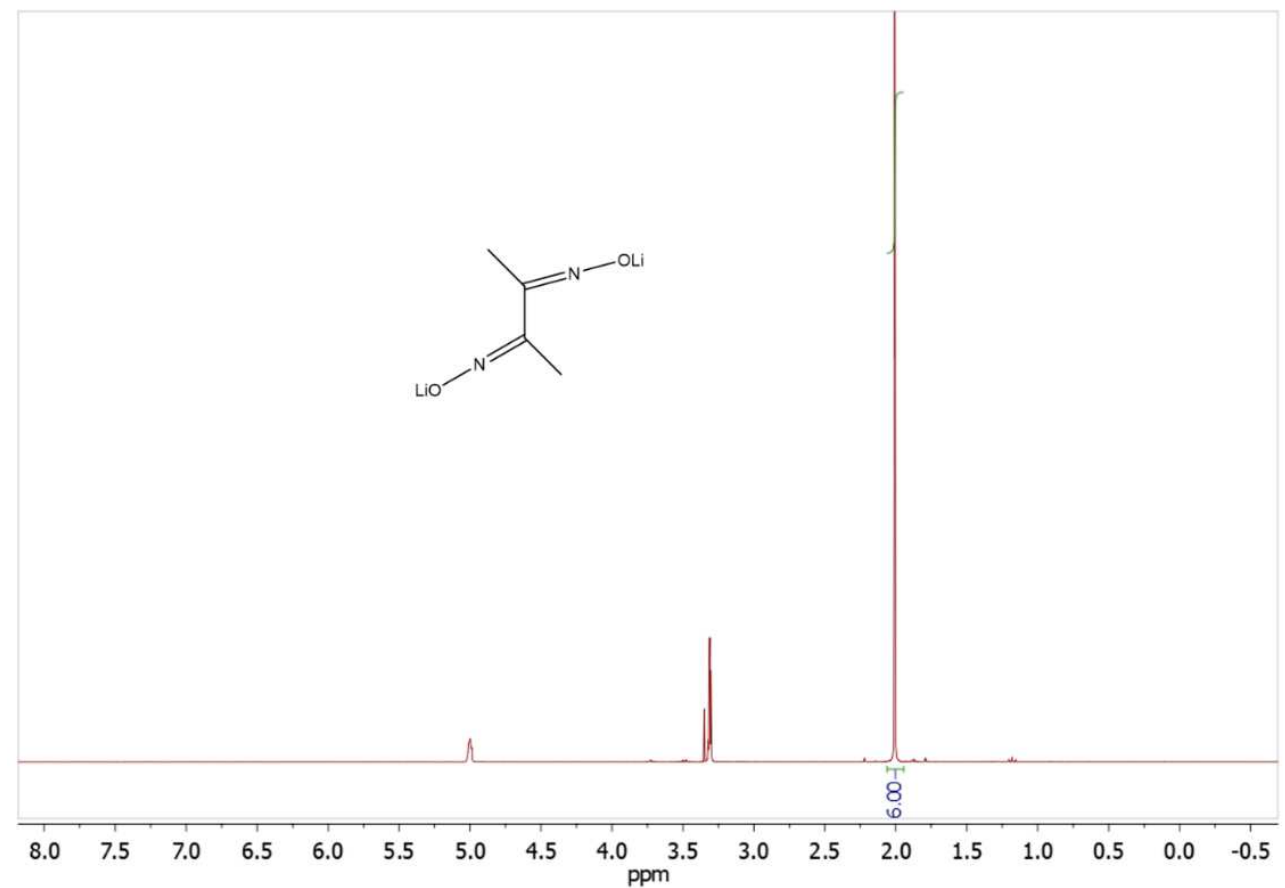


Page $\mid \mathbf{8}$

Supplementary Figure S2. ${ }^{13} \mathrm{C}$ NMR (GeOD) spectrum of $\mathrm{Li}_{2}$-DMGO.

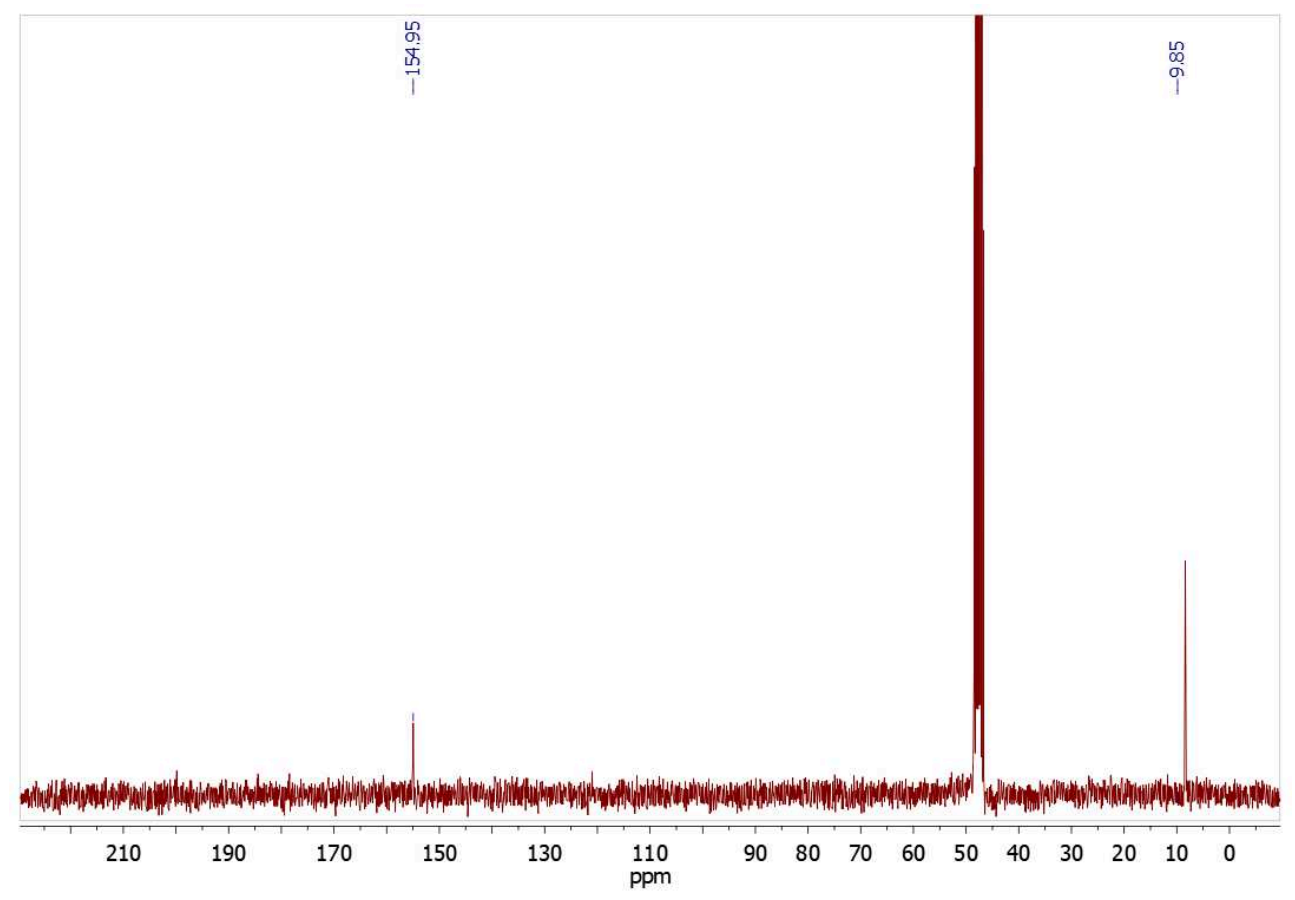




\section{- Dilithium p-Benzoquinone dioxime ( $\left.\mathrm{Li}_{2}-\mathrm{BQDO}\right)$}

p-Benzoquinone dioxime (276 $\mathrm{mg}, 2 \mathrm{mmol}$ ) was dissolved in anhydrous THF $(6 \mathrm{ml})$, and solid lithium hydride ( $32 \mathrm{mg}, 4 \mathrm{mmol}$ ) was added to the solution under stirring. The reaction (performed in an Argon filled glove box) was allowed to stir for $48 \mathrm{~h}$ at room temperature. The reaction mixture was afterwards poured into $6 \mathrm{~mL}$ of anhydrous diethyl ether to precipitate the product, followed by centrifugation and washed with a copious amount of diethyl ether. The product ( $\mathrm{Li}_{2}-\mathrm{BQDO}$ ) was collected and dried under vacuum at $70^{\circ} \mathrm{C}$ for $2 \mathrm{~h}$ and $200{ }^{\circ} \mathrm{C}$ for $12 \mathrm{~h}$.

- ${ }^{1} \mathrm{H}$ NMR (300 MHz, MeOD) $\delta 7.25-7.01(\mathrm{~m}, 2 \mathrm{H}), 6.77-6.55(\mathrm{~m}, 2 \mathrm{H})$.

$-{ }^{13} \mathrm{C}$ NMR $(75 \mathrm{MHz}, \mathrm{MeOD}) \delta 156.17,155.87,130.22,126.21,118.41,115.68 \mathrm{ppm}$.

- HRMS (ESI) calcd for $\mathrm{C}_{6} \mathrm{H}_{4} \mathrm{Li}_{2} \mathrm{~N}_{2} \mathrm{O}_{2}:$ 150.0593, found $151.0350[\mathrm{M}-\mathrm{H}]^{+}$.

- Elemental Analysis: Calculated: C: 48.05\%; H: 2.69\%; N: 18.68\%. Found: C: 47.65\%; H: 3.45\%; $\mathrm{N}: 17.58 \%$.

\section{Supplementary Figure S3. ${ }^{1} \mathrm{H} N \mathrm{NMR}$ (MeOD) spectrum of $\mathrm{Li}_{2}-\mathrm{BQDO}$.}

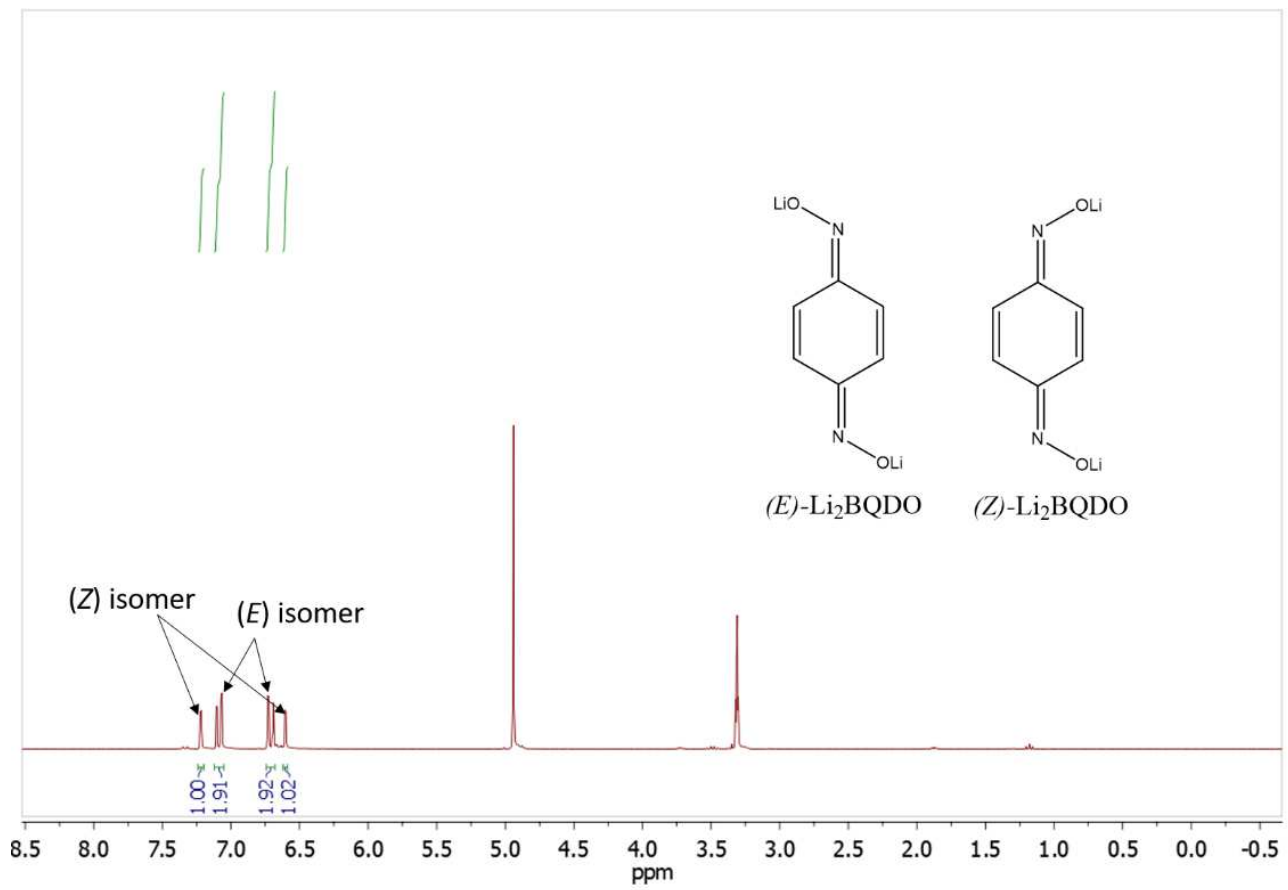




$$
\bar{I}
$$




\section{- Dilithium diphenylglyoxime ( $\mathrm{Li}_{2}$-DPGO)}

Diphenylglyoxime (240 mg, $1 \mathrm{mmol}$ ) was suspended in a mixture of anhydrous tert-butyl alcohol ( $5 \mathrm{ml}$ ) and $\mathrm{MeOH}(5 \mathrm{ml})$, and lithium tert-butoxide (160 mg, $2 \mathrm{mmol}$ ) was added to the suspension under stirring. The reaction (performed in an Argon filled glove box) was kept for $24 \mathrm{~h}$ at room temperature. The light brown precipitate was then filtered and washed with diethyl ether $(10 \mathrm{~mL}) 3$ times. The product ( $\left.\mathrm{Li}_{2}-\mathrm{DPGO}\right)$ was collected and dried at $60{ }^{\circ} \mathrm{C}$, then at $200{ }^{\circ} \mathrm{C}$ for $2 \mathrm{~h}$ under vacuum giving a yield of $90 \%$.

- ${ }^{1} \mathrm{H}$ NMR (300 MHz, MeOD) $\delta$ 7.43-7.37 (m, 4H), 7.23 - $7.16(\mathrm{~m}, 6 \mathrm{H}) \mathrm{ppm}$.

$-{ }^{13} \mathrm{C}$ NMR (75 MHz, MeOD) $\delta$ 156.86, 136.72, 129.24, 129.11, 128.80 ppm.

- HRMS (ESI) calcd for $\mathrm{C}_{14} \mathrm{H}_{10} \mathrm{Li}_{2} \mathrm{~N}_{2} \mathrm{O}_{2} 252.1062$, found 252.9240[M] ${ }^{+}$.

- Elemental Analysis: Calculated: C: 66.69\%; H: 4.00\%; N: 11.11\%. Found: C: 65.67\%; H: 3.77 $\%$; N: $10.79 \%$.

Supplementary Figure S5. ${ }^{1} \mathrm{H}$ NMR (MeOD) spectrum of $\mathrm{Li}_{2}-\mathrm{DPGO}$.

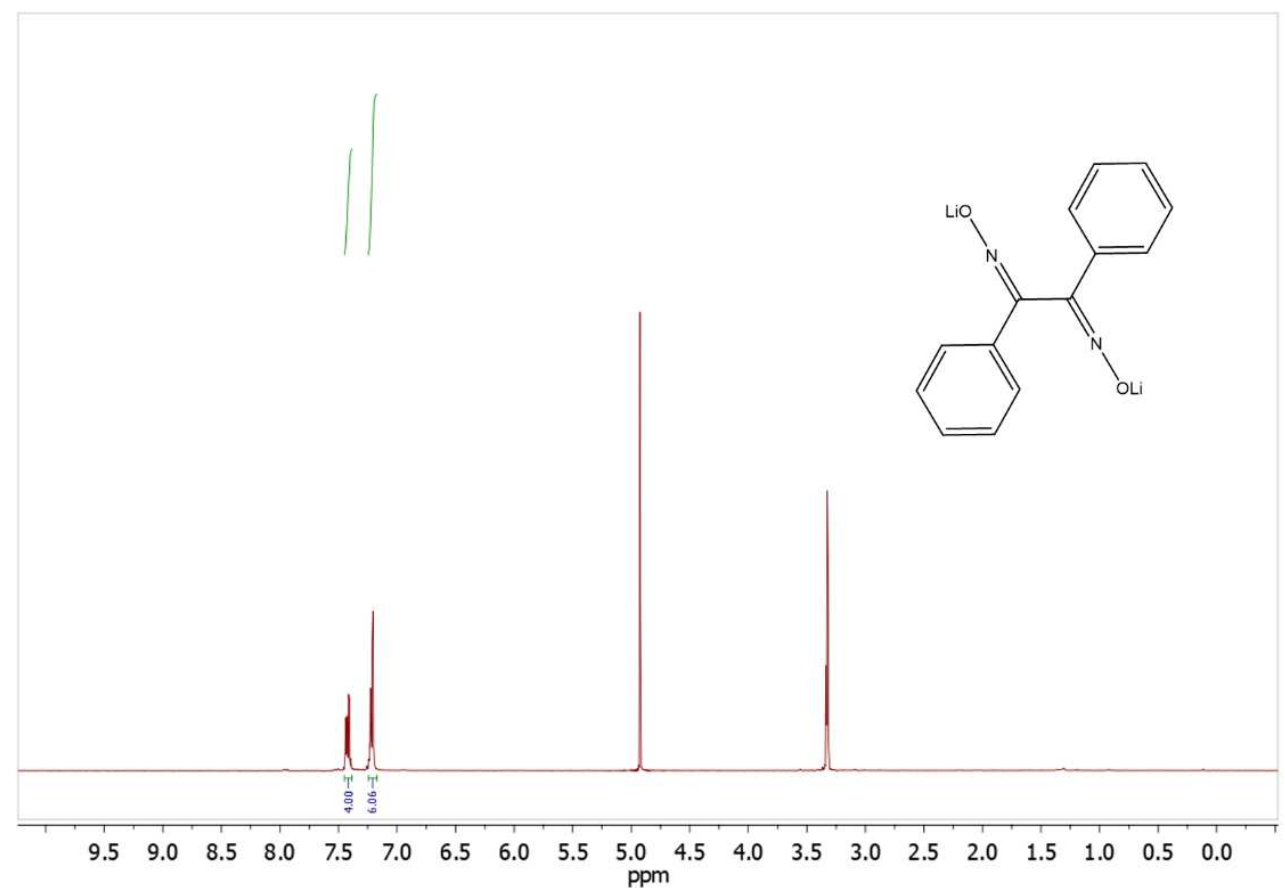


Pag e | $\mathbf{1 2}$

Supplementary Figure S6. ${ }^{13} \mathrm{C}$ NMR (MeOD) spectrum of Li 2 -DPGO.

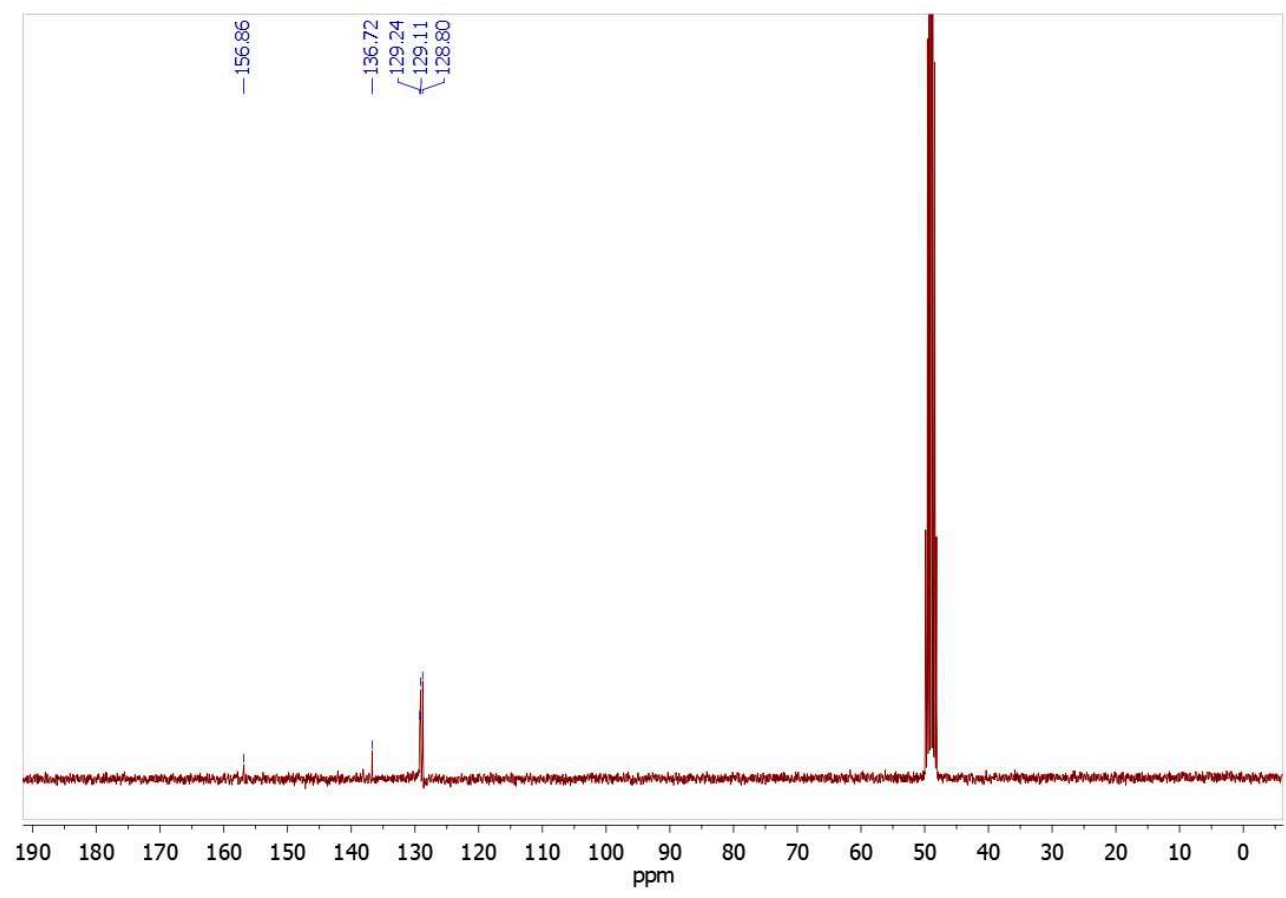


-Dilithium 9,10-phenanthrenedione oximate ( $\left.\mathrm{Li}_{2}-\mathrm{PADO}\right)$

To a suspension of 9,10-phenanthrenedione $(2.08 \mathrm{~g}, 10 \mathrm{mmol}$ ) in EtOH $(50 \mathrm{ml})$, a EtOH (30 $\mathrm{ml}$ ) solution of $\mathrm{NH}_{2} \mathrm{OH} \cdot \mathrm{HCl}(1.63 \mathrm{~g}, 25 \mathrm{mmol})$ and pyridine $(2.03 \mathrm{ml}, 25 \mathrm{mmol})$ was added. The resulting orange suspension was refluxed for $22 \mathrm{~h}$, during which the solids dissolved, and the suspension turned to clear yellow solution. The resulting solution was cooled, filtered, and left for slow evaporation at room temperature. After $24 \mathrm{~h}$, a yellow microcrystalline solid of the dioxime was formed, which was collected by filtration, washed with cold EtOH, and dried under vacuum for $24 \mathrm{~h}$. The yield was $90 \%$.

- ${ }^{1} \mathrm{H}$ NMR (300 MHz, DMSO-d6) $\delta 12.34-12.12(\mathrm{~m}, 2 \mathrm{H}), 8.42-8.47(\mathrm{~m}, 1 \mathrm{H}), 8.04-8.12(\mathrm{~m}, 2 \mathrm{H})$, 7.74-7.83 (dd, J = 7.7, $1.4 \mathrm{~Hz}, 1 \mathrm{H}), 7.38-7.55(\mathrm{~m}, 4 \mathrm{H}) \mathrm{ppm}$.

$-{ }^{13} \mathrm{C}$ NMR (75 MHz, DMSO) $\delta$ 133.22, 132.26, 131.58, 130.76, 130.01, 128.81, 128.17, 127.94, $126.72,125.04,124.94,124.74,124.36$ ppm.

\section{Supplementary Figure S7. ${ }^{1} \mathrm{H}$ NMR (DMSO-d6) spectrum of $\mathrm{H}_{2}$-PADO}

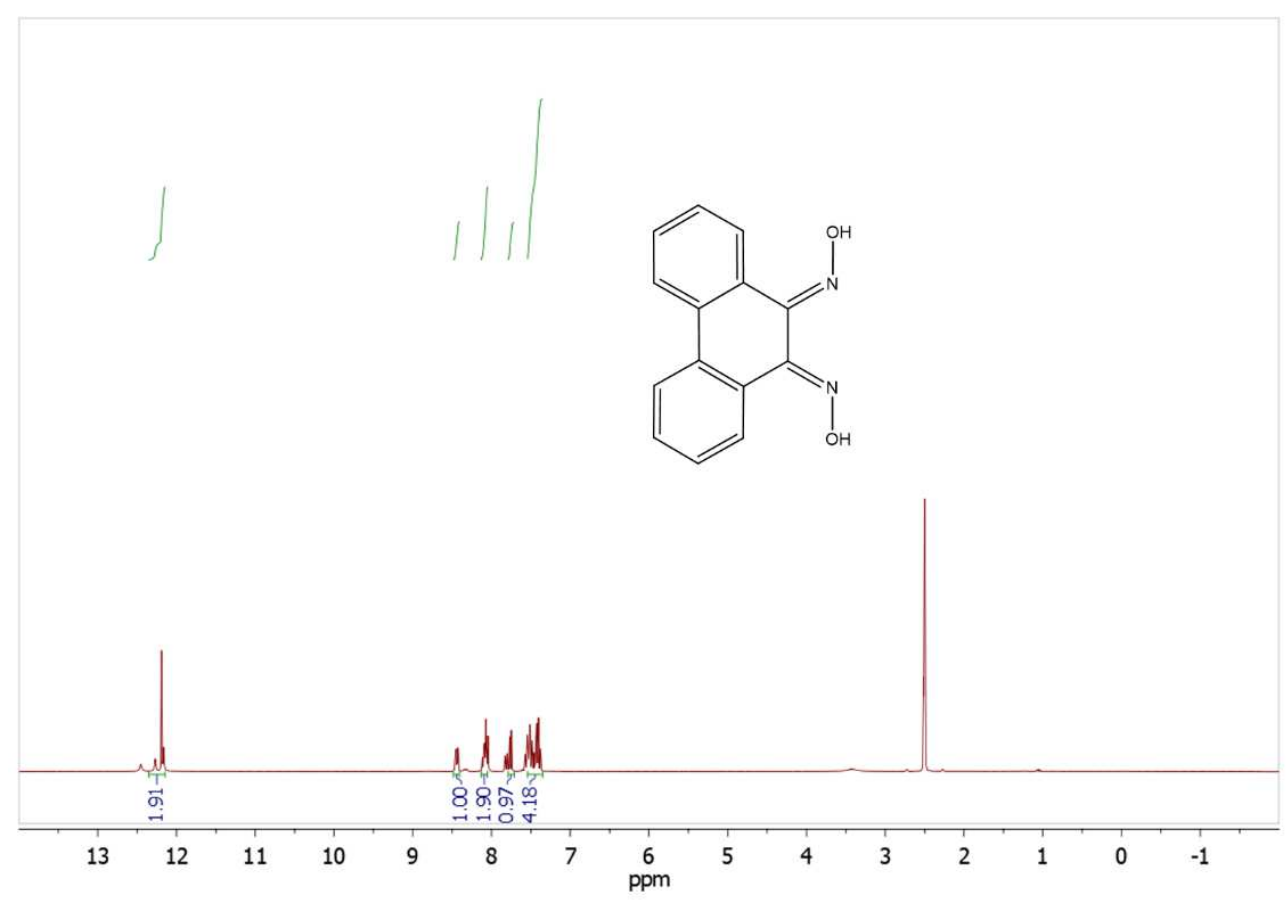




\section{Supplementary Figure S8. ${ }^{13} \mathrm{C}$ NMR (DMSO-d6) spectrum of $\mathrm{H}_{2}$-PADO.}

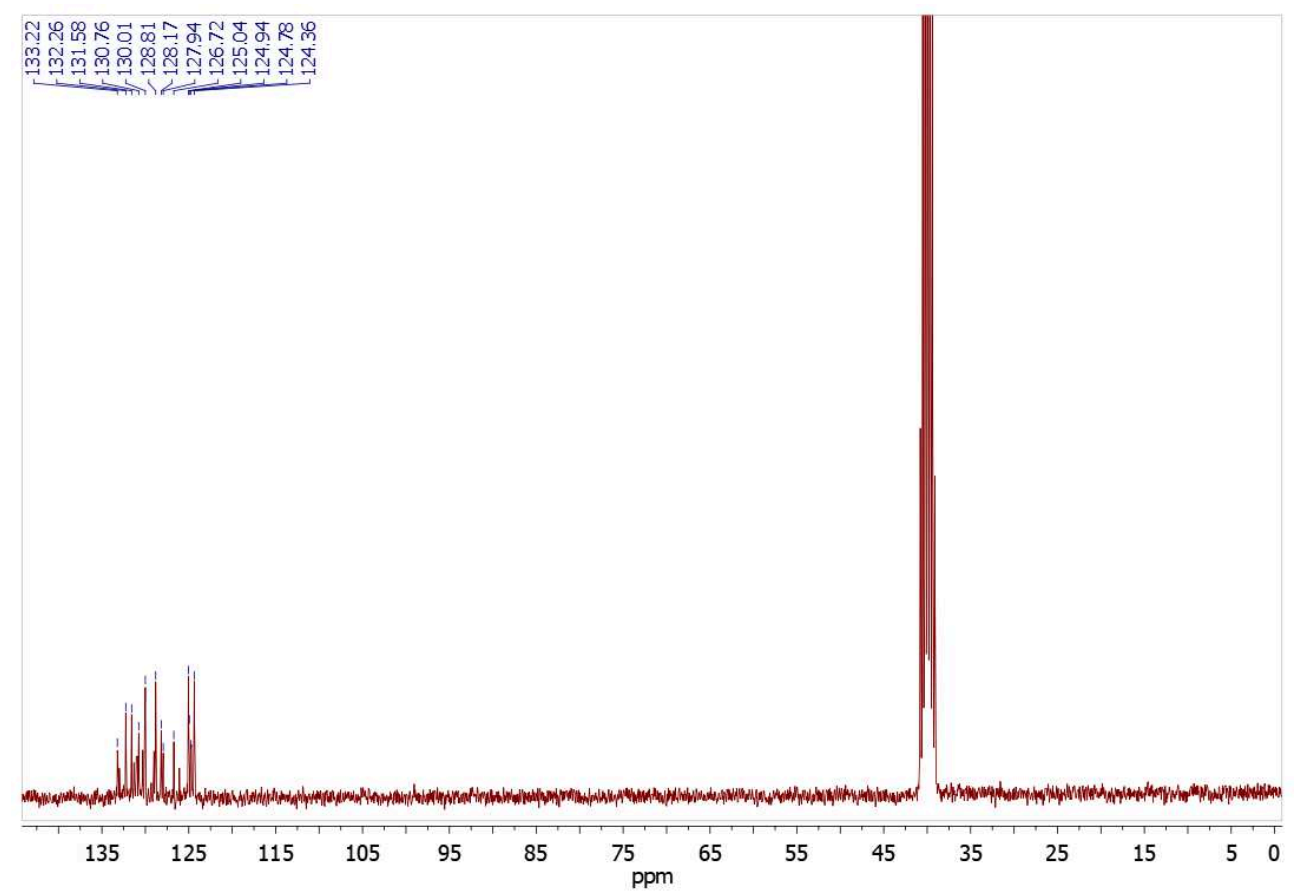

The lithiation was done by suspending $\mathrm{H}_{2}$-PADO $(238 \mathrm{mg}, 1 \mathrm{mmol})$ in $\mathrm{MeOH}(5 \mathrm{ml})$. To the stirred suspension, lithium methoxide $(76 \mathrm{mg}, 2 \mathrm{mmol}$ ) was added. The reaction (performed in an Argon filled glove box) was kept for $24 \mathrm{~h}$ at room temperature. The resulting product was obtained by adding an excess amount of anhydrous diethyl ether $(30 \mathrm{ml})$ to the solution. The product ( $\mathrm{Li}_{2}-\mathrm{PADO}$ ) was collected by filtration, and dried at $60^{\circ} \mathrm{C}$ under vacuum, followed by $200{ }^{\circ} \mathrm{C}$ under vacuum for $3 \mathrm{~h}$.

$-{ }^{1} \mathrm{H}$ NMR (300 MHz, MeOD) $\delta 8.10(\mathrm{dd}, J=7.9,1.4 \mathrm{~Hz}, 2 \mathrm{H}), 8.03(\mathrm{~d}, J=7.6 \mathrm{~Hz}, 2 \mathrm{H}), 7.49-7.42$ (m, 2H), $7.37-7.30(\mathrm{~m}, 2 \mathrm{H})$.

$-{ }^{13}$ C NMR (75 MHz, MeOD) $\delta 152.59,132.87,132.29,130.03,128.83,126.27,124.29$ ppm.

- HRMS (ESI) calcd for $\mathrm{C}_{14} \mathrm{H}_{8} \mathrm{Li}_{2} \mathrm{~N}_{2} \mathrm{O}_{2}: 250.0906[\mathrm{M}-\mathrm{Li}]^{+}$, found $250.9920[\mathrm{M}-\mathrm{H}]^{+}$.

- Elemental Analysis: Calculated: C:67.23\%; H: 3.22\%; N: 11.20\%. Found: C: 66.27\%; H: 3.22\%; $\mathrm{N}: 10.57 \%$. 
Supplementary Figure S9. ${ }^{1} \mathrm{H}$ NMR (MeOD) spectrum of $\mathrm{Li}_{2}-\mathrm{PADO}$.

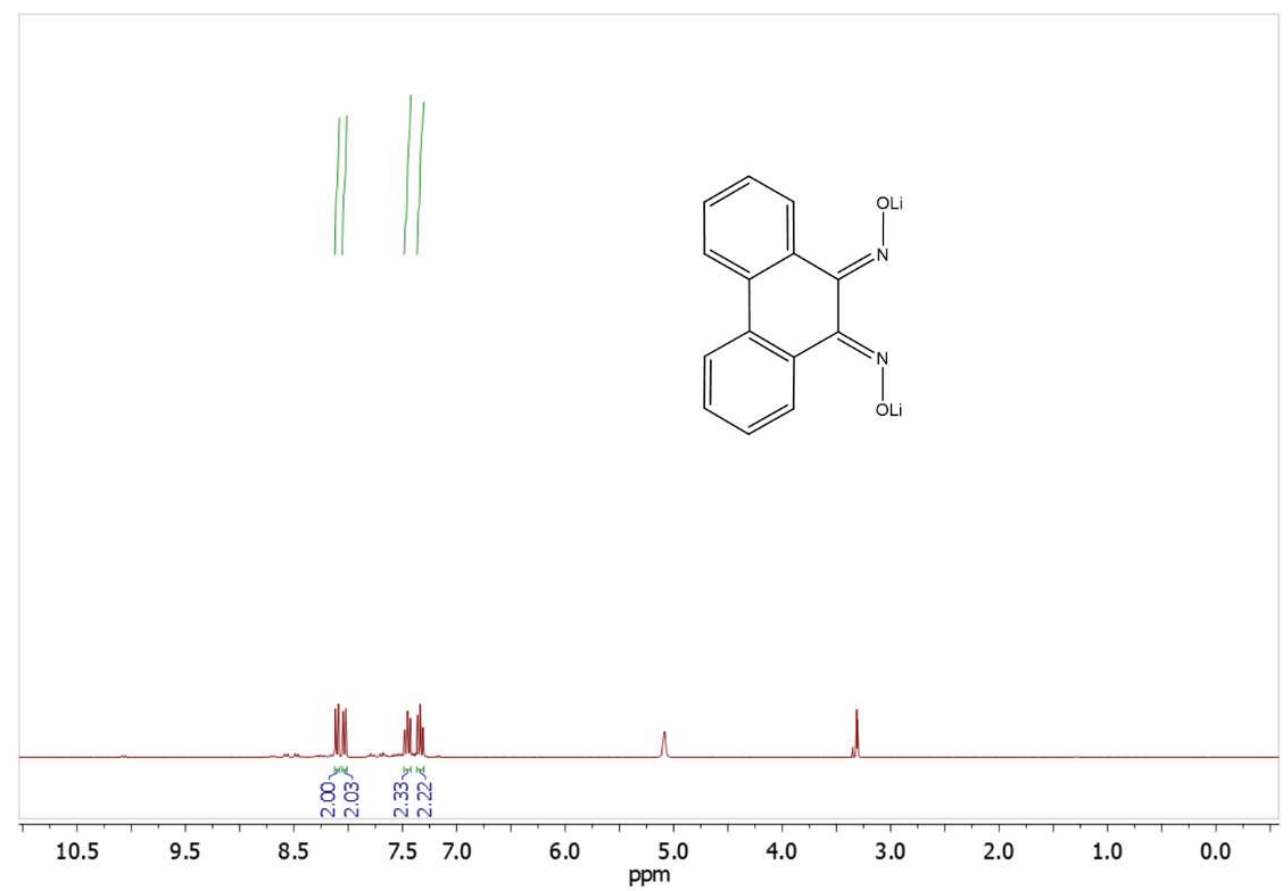

Supplementary Figure S10. ${ }^{13} \mathrm{C}$ NMR (MeOD) spectrum of $\mathrm{Li}_{2}-\mathrm{PADO}$.

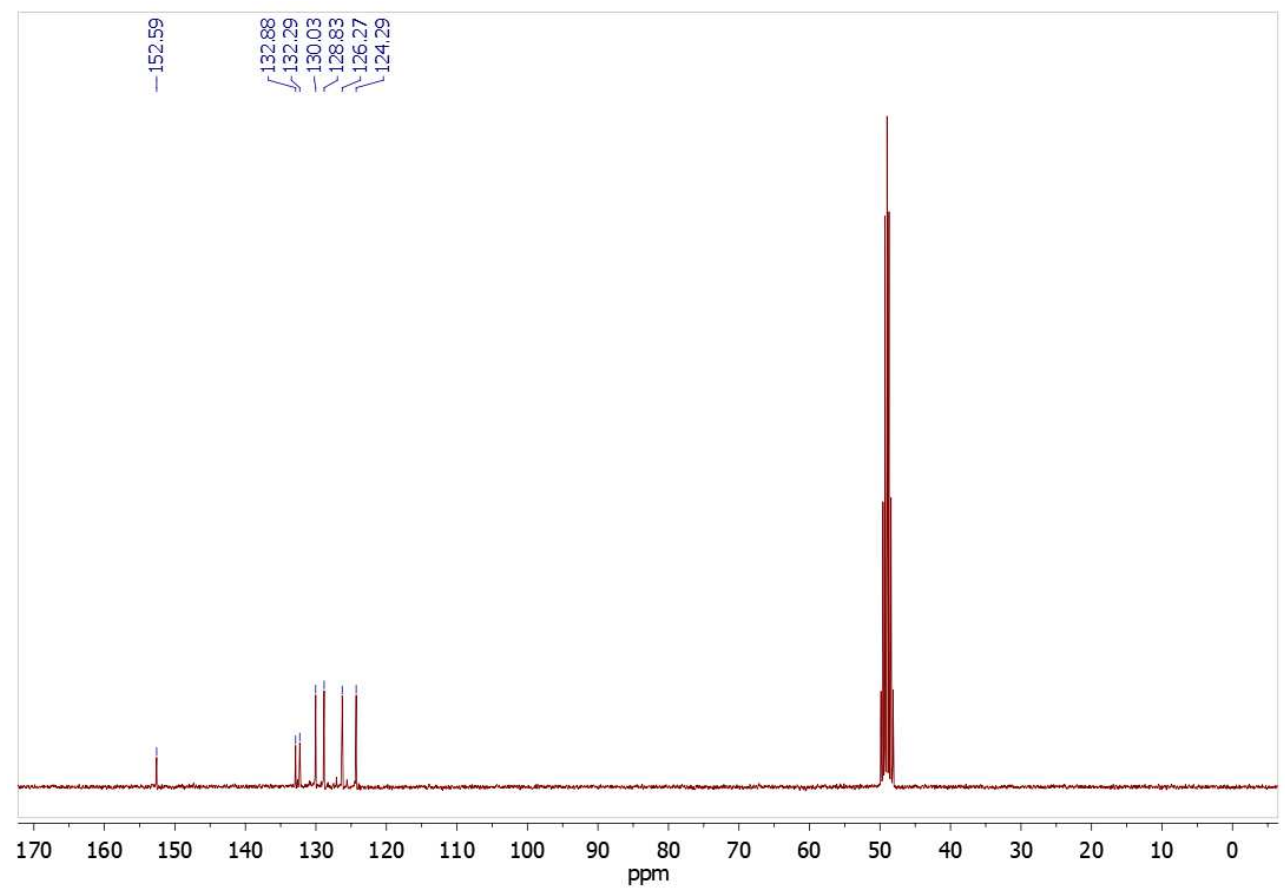


- Tetralithium-1,1,4,4-Tetramethoxy-2,3,5,6-tetraoximatecyclohexane ( $\mathrm{Li}_{4}$-TMTO). ${ }^{3}$

To a solution of phloroglucinol dihydrate $(10 \mathrm{~g}, 0.06 \mathrm{~mol})$ in methanol $(100 \mathrm{~mL}), 0.5 \mathrm{ml}$ of acetic acid was added. The suspension was cooled to $0{ }^{\circ} \mathrm{C}$, and isopropyl nitrite $(22 \mathrm{~mL}, 0.214$ mol) was added dropwise within $1 \mathrm{~h}$, while stirring and keeping the temperature of reaction at $0^{\circ} \mathrm{C}$. The mixture was stirred for another $0.5 \mathrm{~h}$ at room temperature. After that, the solvent was evaporated in vacuo at $35{ }^{\circ} \mathrm{C}$ of the water bath. The crude product was suspended in diethyl ether, a solid was filtered off, washed with diethyl ether, and dried. The material was used for the next step without further purification.

The 2,4,6-Trinitrosobenzene-1,3,5-triol (12.8g, $0.055 \mathrm{~mol})$ obtained above was solubilized in $200 \mathrm{ml}$ of methanol to which $3.84 \mathrm{~g}$ of hydroxylamine hydrochloride was added. The red solution was kept at room temperature for 30 days, afterwards, the white precipitate was filtrated, washed with water, methanol and dried at room temperature. Yield: $11.5 \mathrm{~g}$ of $\mathrm{H}_{4}$ TMTO, 65\%.

- ${ }^{1} \mathrm{H}$ NMR (300 MHz, DMSO-d6): $\delta=3.15$ (6H, s, OMe), 3.41 (6H, s, OMe), $11.53(4 \mathrm{H}, \mathrm{s}, \mathrm{NOH})$ ppm.

- ${ }^{13} \mathrm{C}$ NMR (100 MHz, DMSO-d6): $\delta=50.77$ (OMe), 51.63 (OMe), 100.12 (C(OMe)), 144.95(C=NOH) ppm.

Supplementary Figure S11. ${ }^{1} \mathrm{H}$ NMR and ${ }^{13} \mathrm{C}$ NMR (DMSO-d6) spectra of $\mathrm{H}_{4}$-TMTO.

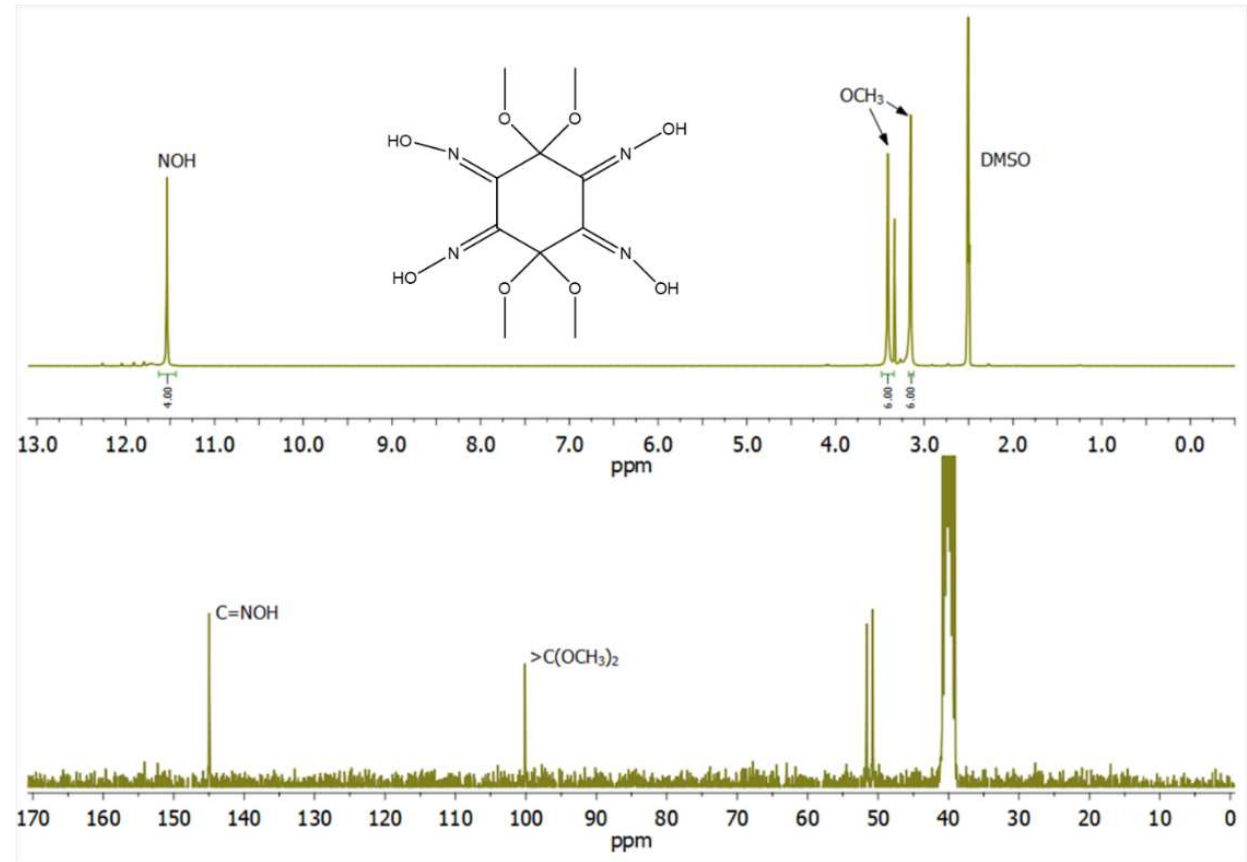


$100 \mathrm{mg}(0.31 \mathrm{mmol})$ of $\mathrm{H}_{4}$-TMTO was suspended in methanol $(9 \mathrm{ml})$ and lithium methoxide $(48.5 \mathrm{mg}, 1.27 \mathrm{mmol})$ was added to the solution under stirring. The reaction (performed in an Argon filled glove box) was kept for $24 \mathrm{~h}$ at room temperature. Then, anhydrous diethyl ether $(30 \mathrm{ml})$ was added to the reaction mixture, resulting in the precipitation of a white product, followed by filtration and washed with diethyl ether 4 times. The product ( $\mathrm{Li}_{4}-\mathrm{TMTO}$ ) was dried at $120^{\circ} \mathrm{C}$ for $12 \mathrm{~h}$ under a vacuum to remove free diethyl ether and methanol. Yield: $80.5 \mathrm{mg}$, $75 \%$.

$-{ }^{1} \mathrm{H}$ NMR (300 MHz, MeOD): $\delta=3.32(6 \mathrm{H}, \mathrm{s}, \mathrm{OMe}) \mathrm{ppm}$.

$-{ }^{13} \mathrm{C}$ NMR (75 MHz, MeOD): $\delta=51.60$ (OMe), 101.31 (C(OMe)), 152.34(C=NOH) ppm.

- HRMS (ESI) calcd for $\mathrm{C}_{10} \mathrm{H}_{12} \mathrm{Li}_{4} \mathrm{~N}_{4} \mathrm{O}_{8}: 344.1295$, found: $345.0100[\mathrm{M}-\mathrm{H}]^{+}$.

- Elemental Analysis: Calculated: C:34.92\%; H: 3.52\%; N: 16.29\%. Found: C: 33.40\%; H: 3.73\%; $\mathrm{N}: 15.92 \%$.

\section{Supplementary Figure S12. ${ }^{1} \mathrm{H}$ NMR (MeOD) spectrum of $\mathrm{Li}_{4}-\mathrm{TMTO}$.}

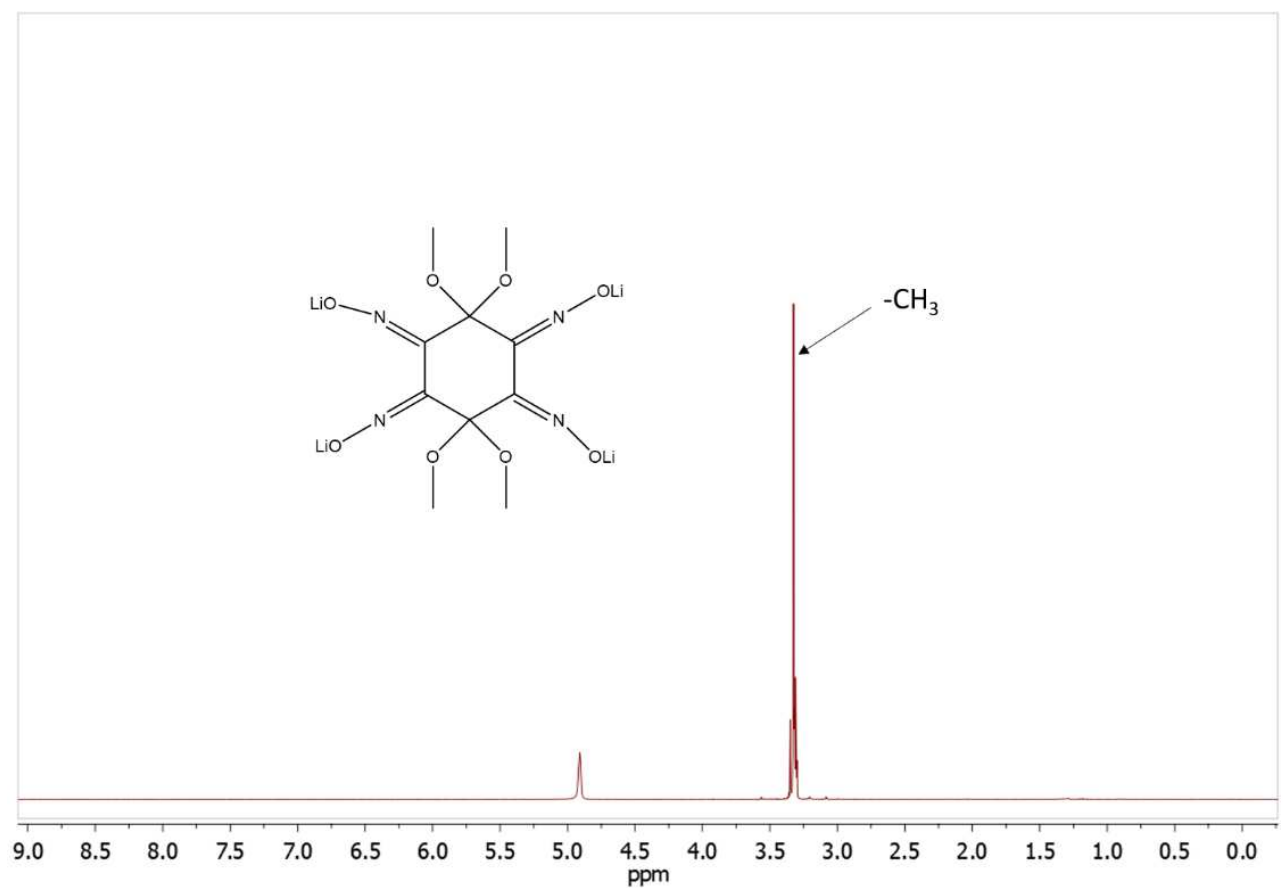


Pa ge | $\mathbf{1 8}$

Supplementary Figure S13. ${ }^{13} \mathrm{C}$ NMR (GeOD) spectrum of $\mathrm{Li}_{4}$-TITO.

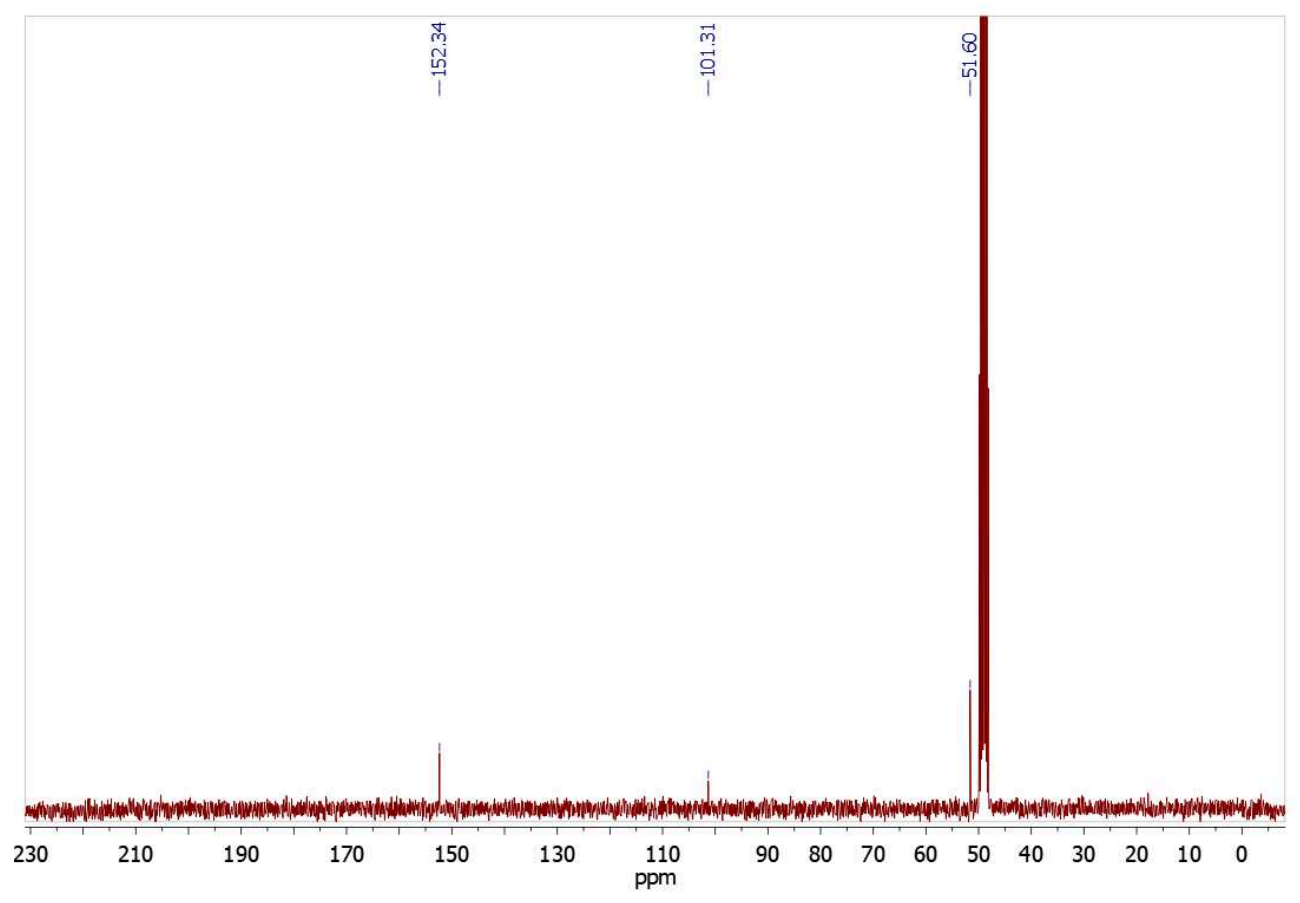




\section{- Poly(1,4-phenyleneazine-N,N-dioxide) (PNND) ${ }^{4}$}

To a stirred solution of $1.38 \mathrm{~g} \mathrm{p}$-benzoquinone dioxime $(10 \mathrm{mmol})$ and $0.84 \mathrm{~g}$ sodium hydroxide ( $21 \mathrm{mmol}$ ) in $30 \mathrm{ml}$ water, $30 \mathrm{ml}$ of $5.5 \%$ sodium hypochlorite (excess amount) was slowly added. The solution turned yellow, and a yellow precipitate gradually formed. Stirring was continued for $1 \mathrm{~h}$. The precipitate was filtered and washed with water 3 times and acetone, followed by drying under vacuum at RT to form PNND, with a yield of $65 \%$.

- Elemental Analysis: Calculated: C: 52.95\%; H: 2.93\%; N: 20.58\%. Found: C: 52.70\%; H: 2.91\%; $\mathrm{N}: 19.46 \%$. 


\section{Crystal structure determination by X-ray powder diffraction method.}

The crystal structure of PNND was solved from the powder XRD data. Powder X-ray diffraction (PXRD) patterns were collected on a STOE Stadi P diffractometer in transmission geometry equipped with a $\mathrm{Cu}$ anticathode (K $\alpha$ radiation, $\lambda=1.540600 \AA$, operating at $50 \mathrm{kV}$ $40 \mathrm{~mA}$ ). For the structure determination from PXRD, we followed the following steps: pattern indexing, space group determination, structure solution and the Rietveld refinement. The indexing procedure was carried out in EXPO2014 ${ }^{5}$, and FullProf suite ${ }^{6}$ using NTREOR, DICVOL. By these indexing procedures a monoclinic system was obtained with high figure of merit (F.O.M.). Space group determination, structure solution and Rietveld refinement were performed in EXPO2014.

Supplementary Table S1. Powder XRD data and refinement parameters of PNND

\begin{tabular}{|c|c|}
\hline \multicolumn{2}{|c|}{ Powder data } \\
\hline Formula & $\mathrm{C}_{3} \mathrm{H}_{2} \mathrm{NO}$ \\
\hline Powder Data temperature (K) & 295 \\
\hline$M_{r}$ & 68.055 \\
\hline crystal system & Monoclinic \\
\hline space group & $P 2_{1} / n$ \\
\hline$a(\AA)$ & $6.394(2)$ \\
\hline$b(\AA)$ & $11.322(4)$ \\
\hline$c(\AA)$ & $3.7072(12)$ \\
\hline$b$ (deg.) & $92.923(8)$ \\
\hline$V\left(\AA^{3}\right)$ & $268.03(15)$ \\
\hline 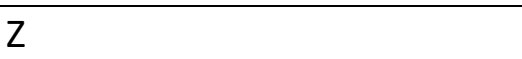 & 4 \\
\hline$\rho\left(\mathrm{g} \mathrm{cm}^{-3}\right)$ & 1.687 \\
\hline \multicolumn{2}{|c|}{ Data collection } \\
\hline$\lambda(\AA)$ & 1.540600 \\
\hline$q_{\min }-q_{\max }($ deg.) & $5-70$ \\
\hline $2 q$ step (deg.) & 0.015 \\
\hline \multicolumn{2}{|c|}{ Refinement } \\
\hline Profile function & Pseudo-Voigt \\
\hline$R_{\mathrm{p}}$ & 2.27 \\
\hline$R_{\mathrm{wp}}$ & 4.76 \\
\hline$R_{\mathrm{e}}$ & 0.992 \\
\hline $\mathrm{Chi}^{2}\left(\mathrm{X}^{2}\right)$ & 22.97 \\
\hline$R$ (Bragg) & 18.11 \\
\hline$R$ (structure factor) & 13.49 \\
\hline
\end{tabular}


Supplementary Table S2. Fractional Atomic Coordinates $(\times 104)$ and Equivalent Isotropic Displacement Parameters $(\AA 2 \times 103)$ for PNND. $U_{\text {eq }}$ is defined as $1 / 3$ of the trace of the orthogonalised $U_{I J}$ tensor.

\begin{tabular}{|l|r|r|r|r|}
\hline Atom & $x$ & $y$ & $z$ & U(eq) \\
\hline C9 & 5947 & 8903 & 10997 & $0(6)$ \\
\hline C10 & 7126 & 9820 & 9598 & $0(7)$ \\
\hline C11 & 6179 & 10897 & 8582 & $0(6)$ \\
\hline N4 & 9303 & 9641 & 9188 & $0(4)$ \\
\hline O2 & 9886 & 8732 & 7066 & $150(6)$ \\
\hline
\end{tabular}

Supplementary Table S3. Bond Lengths for PNND.

\begin{tabular}{|c|c|c|c|c|c|}
\hline Atom & Atom & Length/Å & Atom & Atom & Length/Å \\
\hline C9 & C10 & \multicolumn{2}{|c|}{1.398377 C11 } & $C 9^{1}$ & 1.394392 \\
\hline C9 & $\mathrm{C} 11^{1}$ & \multicolumn{2}{|c|}{1.394390 N4 } & $\mathrm{N} 4^{2}$ & 1.328292 \\
\hline C10 & C11 & \multicolumn{2}{|c|}{$1.405053 \mathrm{~N} 4$} & $\mathrm{O} 2$ & 1.358861 \\
\hline C10 & N4 & \multicolumn{2}{|c|}{1.421947} & & \\
\hline
\end{tabular}

Supplementary Table S4. Bond Angles for PNND.

\begin{tabular}{|c|c|c|c|c|c|c|c|}
\hline Atom & Atom & Atom & Angle/ ${ }^{\circ}$ & Atom & Atom & Atom & Angle $/^{\circ}$ \\
\hline C10 & C9 & $\mathrm{C} 11^{1}$ & 117.93 & C10 & C11 & $\mathrm{CO}^{1}$ & 121.36 \\
\hline C9 & C10 & C11 & 120.70 & C10 & N4 & $\mathrm{N} 4^{2}$ & 120.15 \\
\hline C9 & C10 & N4 & 118.89 & C10 & N4 & $\mathrm{O} 2$ & 117.95 \\
\hline C11 & C10 & N4 & 120.40 & $\mathrm{~N} 4^{2}$ & N4 & 02 & 121.89 \\
\hline
\end{tabular}




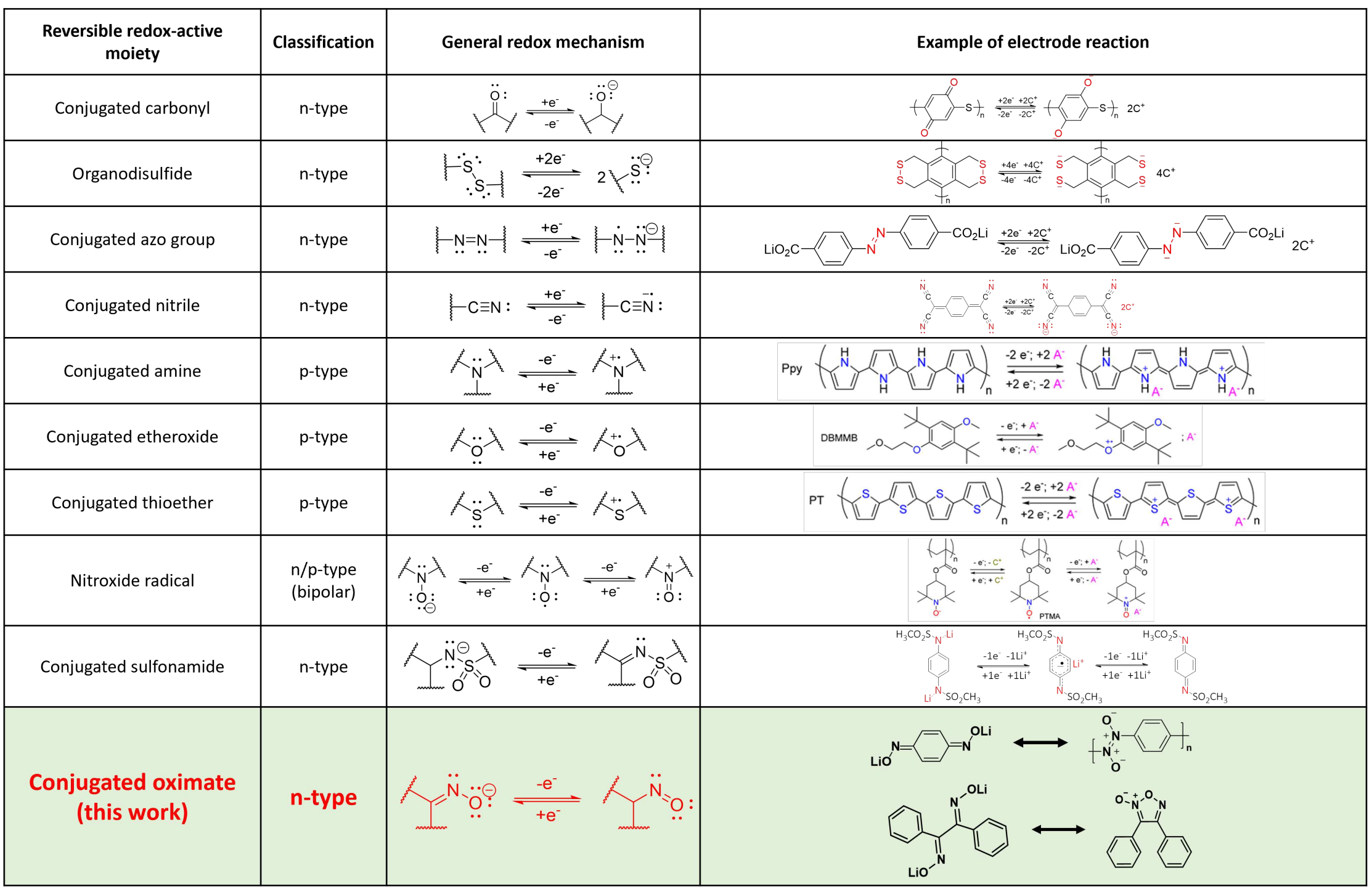

Supplementary Table S5. Reaction mechanisms and groups for electrochemical storage in reported redox-active organic materials, compared to the oximate chemistry disclosed in this work. Table adapted from reference ${ }^{7}$. 

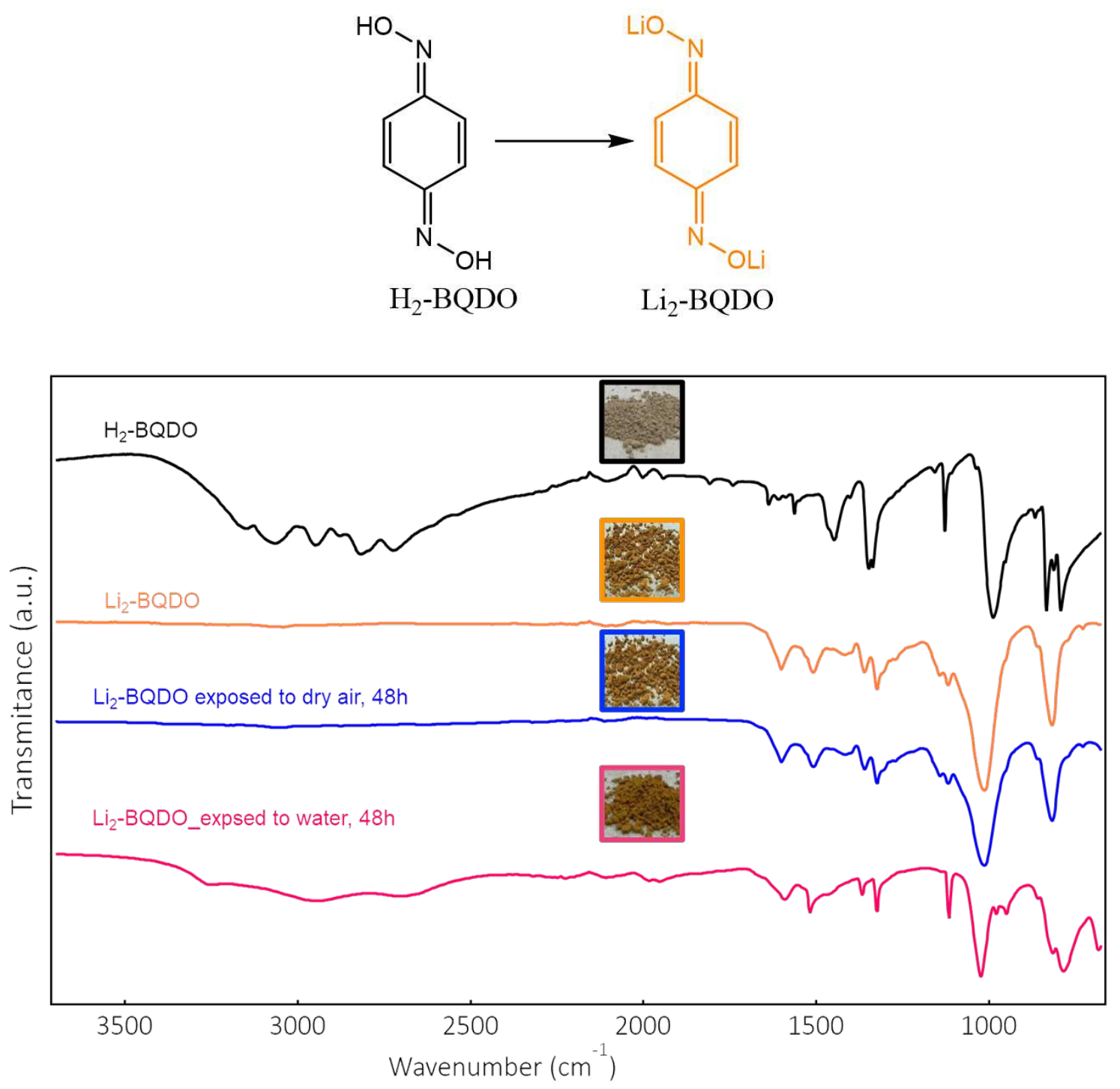

Supplementary Figure S14. FTIR analysis survey for the synthesis of $\mathrm{Li}_{2}-\mathrm{BQDO}$, coupled to air and moisture stability analysis.

The formation of lithiated product ( $\mathrm{Li}_{2}-\mathrm{BQDO}$, orange curve) is confirmed by the disappearance of the weak broad band around $3000 \mathrm{~cm}^{-1}$ attributed to the hydroxyl groups of $\mathrm{H}_{2}$-BQDO (black curve). After lithiation, the broad - $\mathrm{OH}$ band completely disappears (orange curve).

Comparative FTIR spectra of pristine and dry air-exposed (for $48 \mathrm{~h}$ ) samples of $\mathrm{Li}_{2}-\mathrm{BQDO}$ (blue curve) and water vapours exposed (for $48 \mathrm{~h}$ ) Li 2 -BQDO (pink curve). The materials show identical FTIR signatures after dry air-exposure without any sign of decomposition, indicating excellent oxygen tolerance. For water vapours exposed $\mathrm{Li}_{2}$-DPGO (pink curve), residual water or partial hydrolysis can be observed with the characteristic peaks still preserved after $48 \mathrm{~h}$ of exposure. 

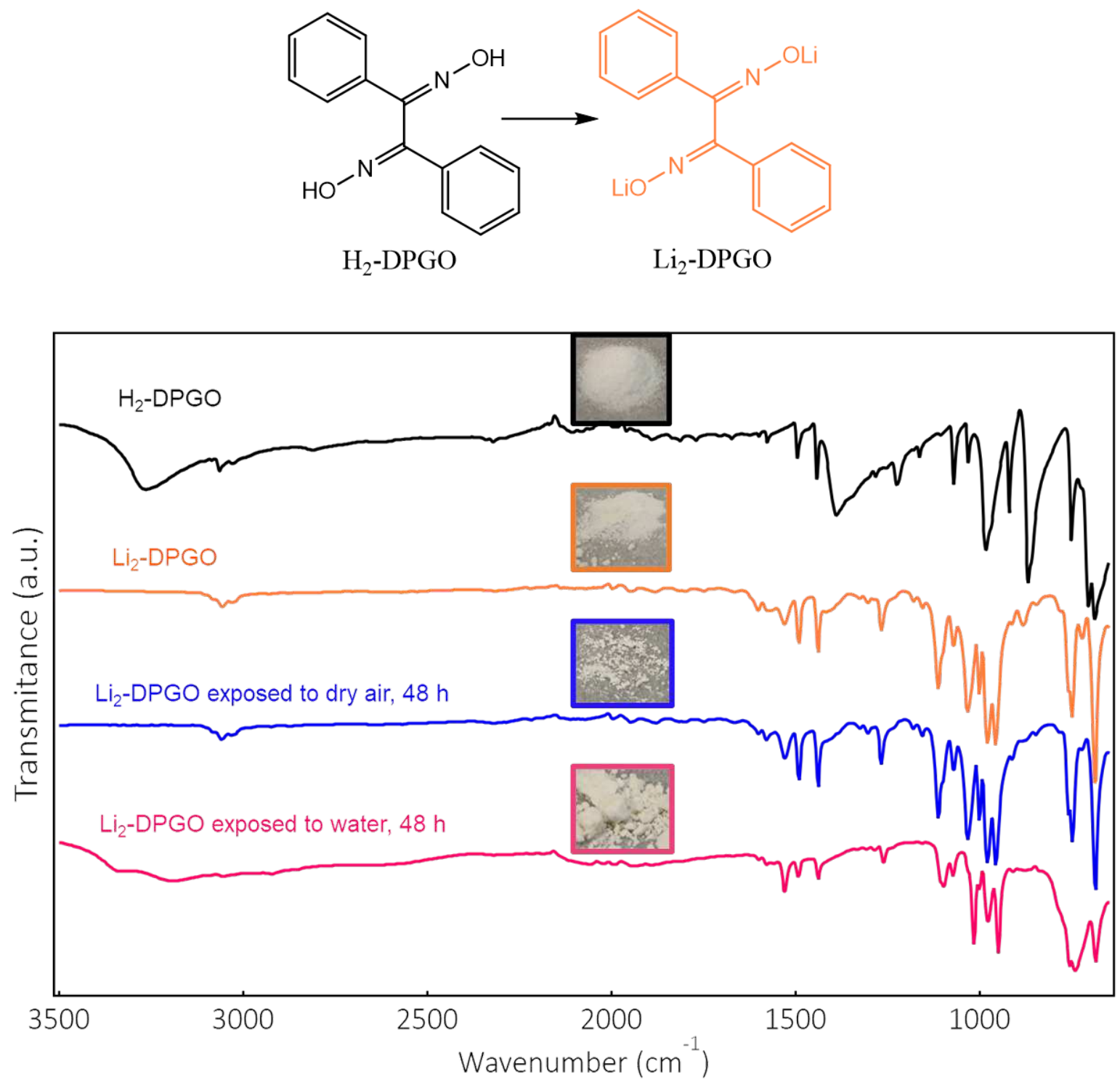

Supplementary Figure S15. FTIR analysis survey for the synthesis of $\mathrm{Li}_{2}$-DPGO, coupled to air and moisture stability analysis.

The formation of pure $\mathrm{Li}_{2}$-DPGO (orange curve) after lithiation of $\mathrm{H}_{2}$-DPGO (black curve) is confirmed by the disappearance of weak and broad O-H stretch band between $3200-3300 \mathrm{~cm}^{-}$ 1 .

Comparative FTIR spectra of pristine and dry air-exposed (for $48 \mathrm{~h}$ ) samples of $\mathrm{Li}_{2}$-DPGO (blue curve) and water vapours exposed $\mathrm{Li}_{2}$-DPGO (pink curve, for $48 \mathrm{~h}$ ). The materials show identical FTIR signatures after dry air-exposure without any sign of decomposition. For water vapour exposed $\mathrm{Li}_{2}$-DPGO (pink curve), residual water or partial hydrolysis can be observed with the characteristic peaks still preserved after $48 \mathrm{~h}$ of exposure. 


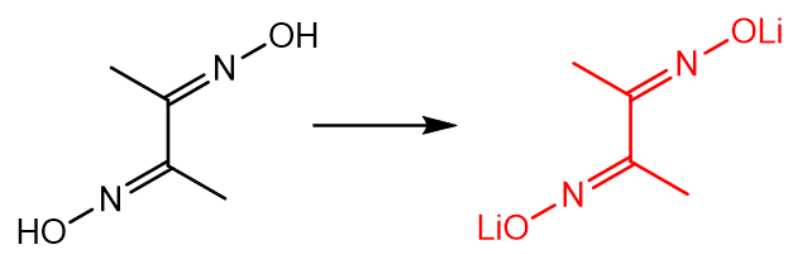

$\mathrm{H}_{2}$-DMGO

$\mathrm{Li}_{2}$-DMGO

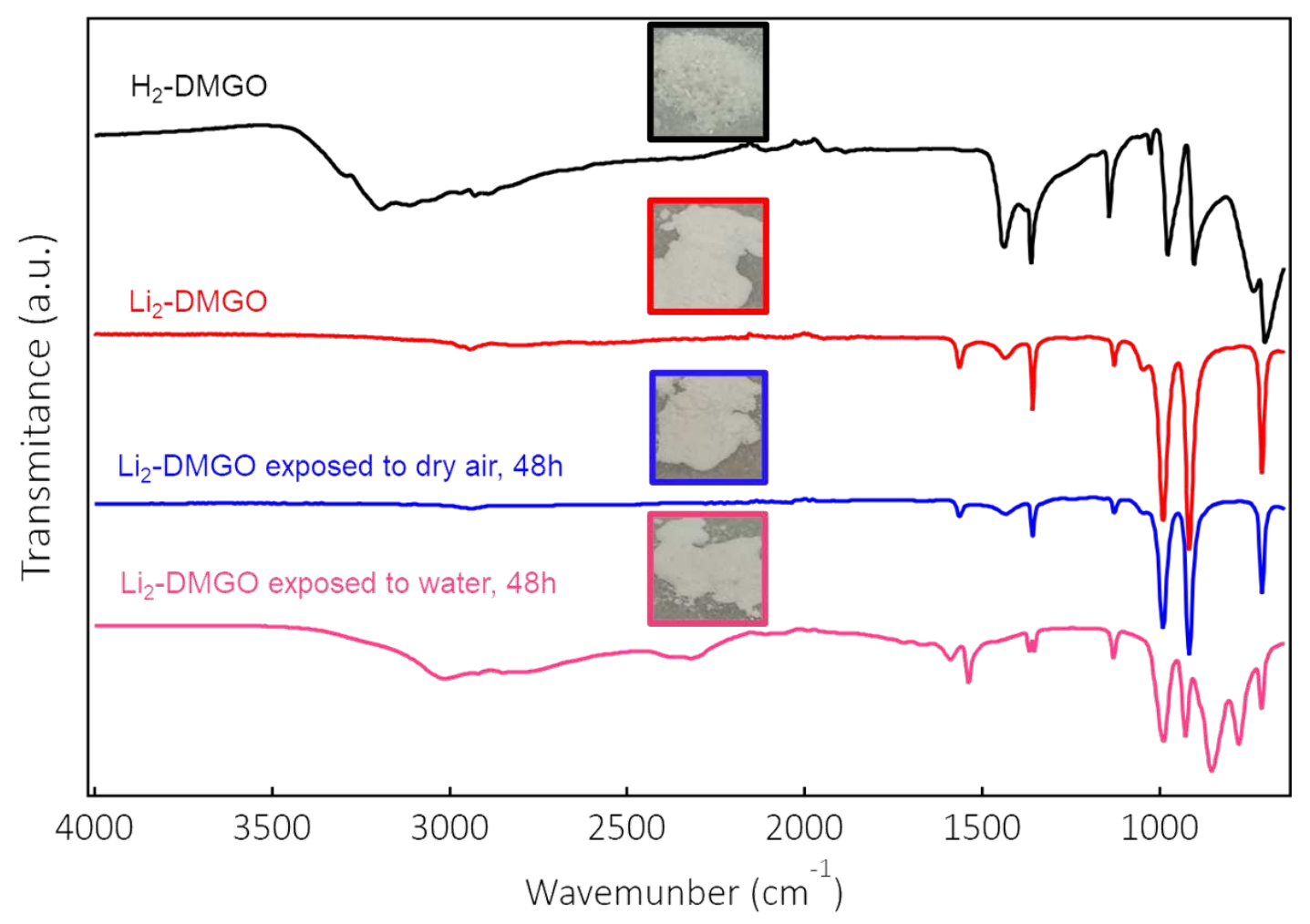

Supplementary Figure S16. FTIR analysis survey for the synthesis of $\mathrm{Li}_{2}$-DMGO, coupled to air and moisture stability analysis.

The formation of lithiated product ( $\mathrm{Li}_{2}-\mathrm{DMGO}$, red curve) is confirmed by the disappearance of the weak broad band between $3000-3400 \mathrm{~cm}^{-1}$ attributed to the hydroxyl groups of $\mathrm{H}_{2}$-DMGO (black curve).

Comparative FTIR spectra of pristine and dry air-exposed (for $48 \mathrm{~h}$ ) samples of $\mathrm{Li}_{2}-\mathrm{DMGO}$ show identical FTIR signatures after dry air-exposure without any sign of decomposition (oxidation nor hydrolysis). For water vapour exposed Li2-DMGO (pink curve), residual water or partial hydrolysis can be observed with the characteristic peaks still preserved after $48 \mathrm{~h}$ of exposure. 

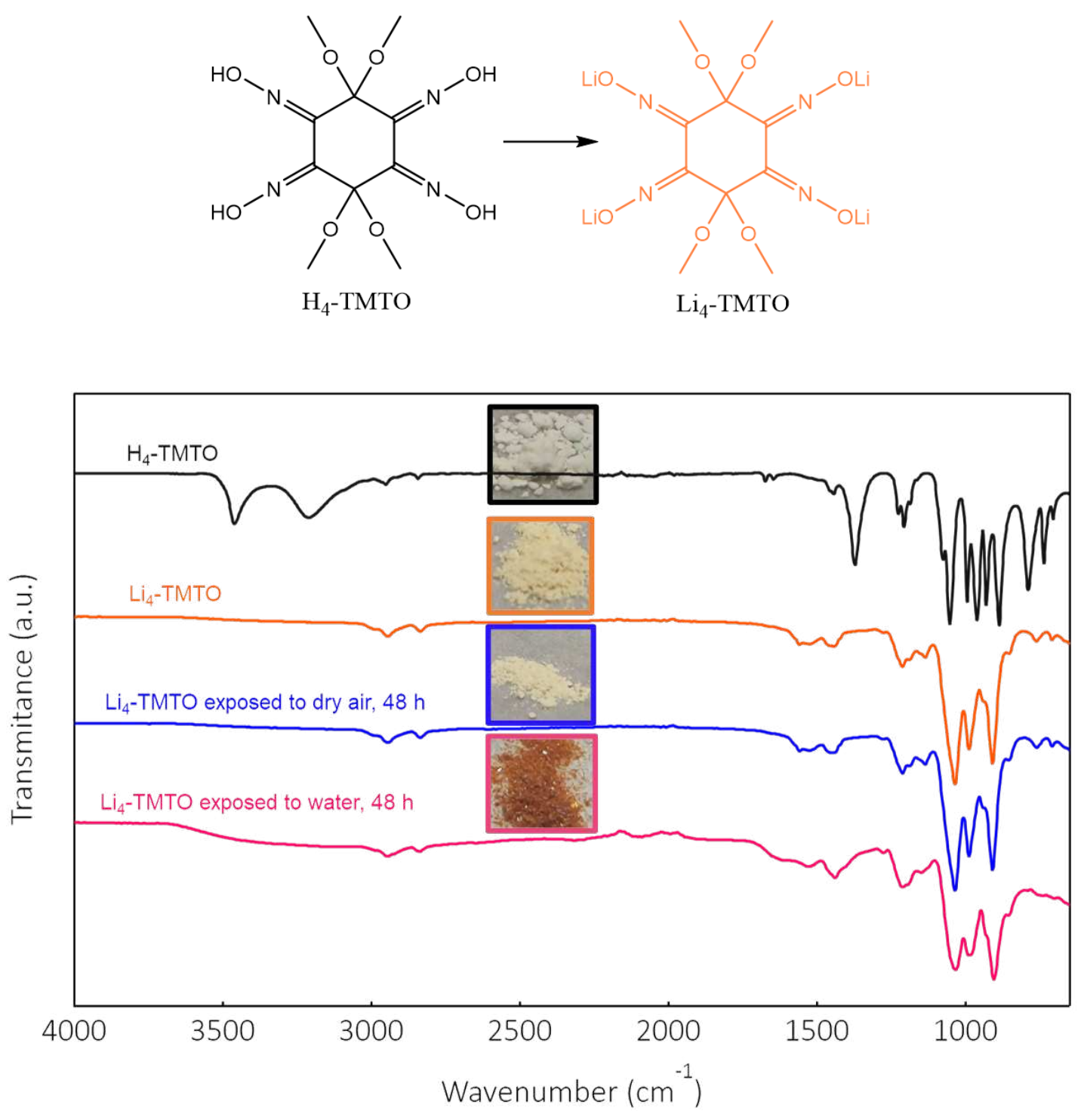

Supplementary Figure S17. FTIR analysis survey for the synthesis of $\mathrm{Li}_{4}-\mathrm{TMTO}$, coupled to air and moisture stability analysis.

The formation of lithiated product ( $\mathrm{Li}_{4}-\mathrm{TMTO}$, orange curve) is confirmed by the disappearance of the two broad bands between $3000-3500 \mathrm{~cm}^{-1}$ attributed to the hydroxyl group of $\mathrm{H}_{4}$-TMTO (black curve).

Comparative FTIR spectra of pristine and dry air-exposed (for $48 \mathrm{~h}$ ) samples of $\mathrm{Li}_{4}$-TMTO show identical FTIR signatures (blue curve) after dry air-exposure, without any sign of decomposition (oxidation nor hydrolysis). For water vapour exposed $\mathrm{Li}_{2}$-DPGO (pink curve), residual water or partial hydrolysis can be observed with the characteristic peaks still preserved after $48 \mathrm{~h}$ of exposure. 


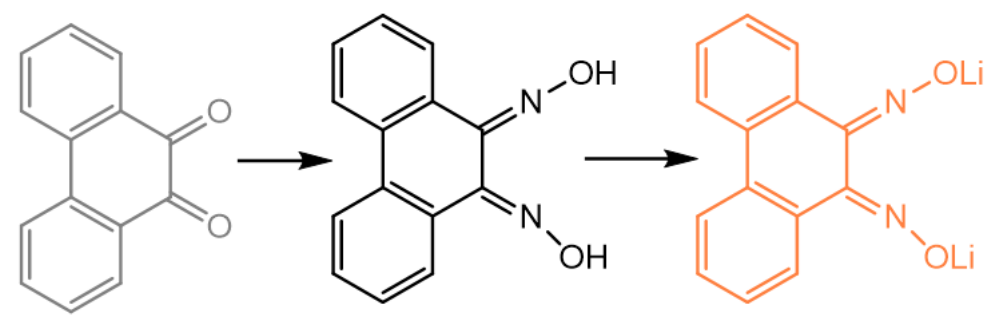

9,10 phenanthrenequinone $\quad \mathrm{H}_{2}$-PADO $\quad \mathrm{Li}_{2}$-PADO

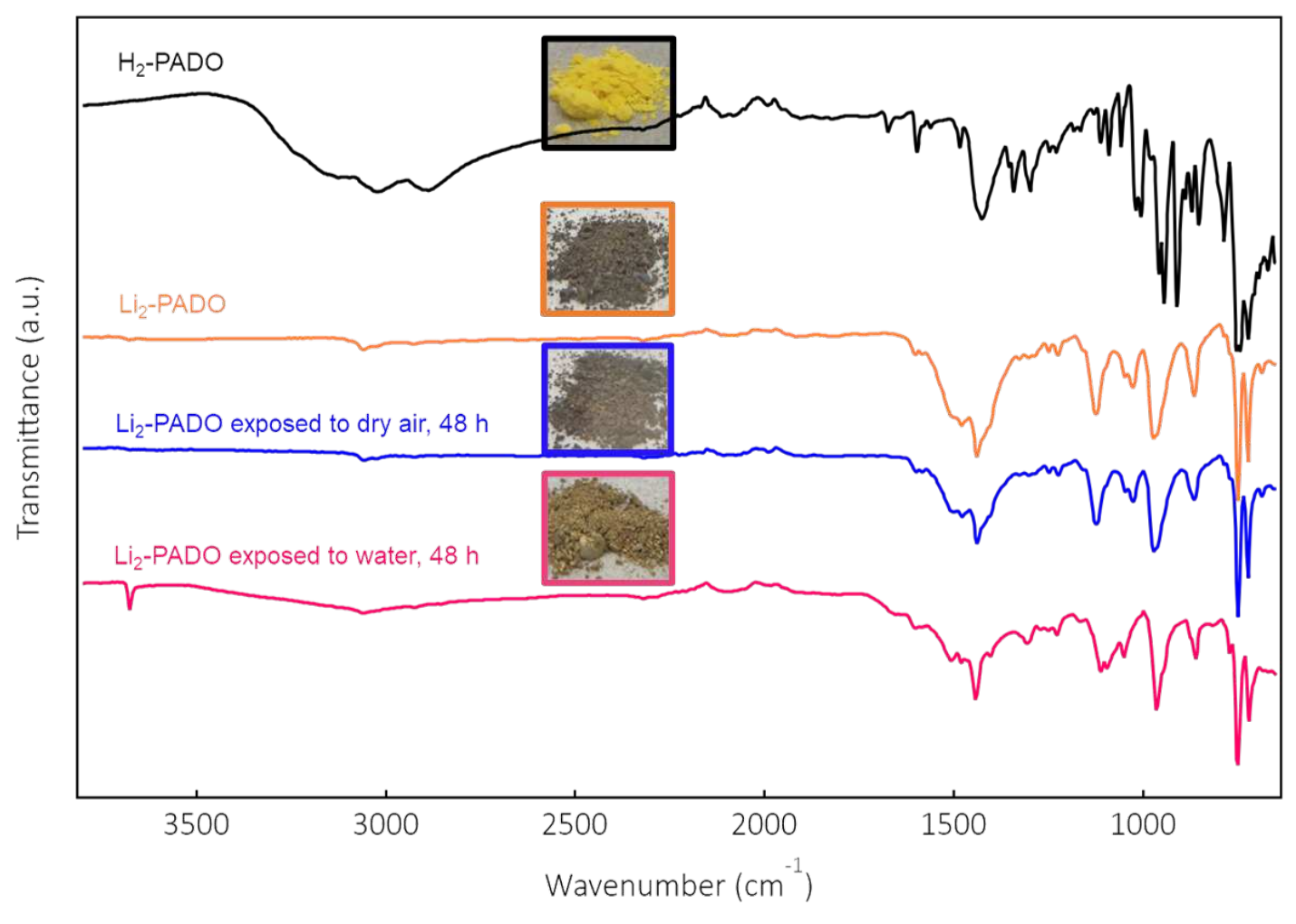

Supplementary Figure S18. FTIR analysis survey for the synthesis of $\mathrm{Li}_{2}-\mathrm{PADO}$, coupled to air and moisture stability analysis.

The formation of lithiated product ( $\mathrm{Li}_{2}-\mathrm{PADO}$, orange curve) is confirmed by the disappearance of the broad band between $3000-3500 \mathrm{~cm}^{-1}$ attributed to the hydroxyl group of $\mathrm{H}_{2}$-PADO (black curve).

Comparative FTIR spectra of pristine and dry air-exposed (for $48 \mathrm{~h}$ ) samples of $\mathrm{Li}_{2}$-PADO show identical FTIR signatures after dry air-exposure without any sign of decomposition (oxidation nor hydrolysis) proving stability under dry conditions as well as high oxidation potential (> $2.91 \mathrm{~V}$ vs. $\mathrm{Li}^{+} / \mathrm{Li}$ ). For water vapour exposed $\mathrm{Li}_{2}-\mathrm{PADO}$ (pink curve), residual water or partial hydrolysis can be observed with the characteristic peaks still preserved after $48 \mathrm{~h}$ of exposure. 


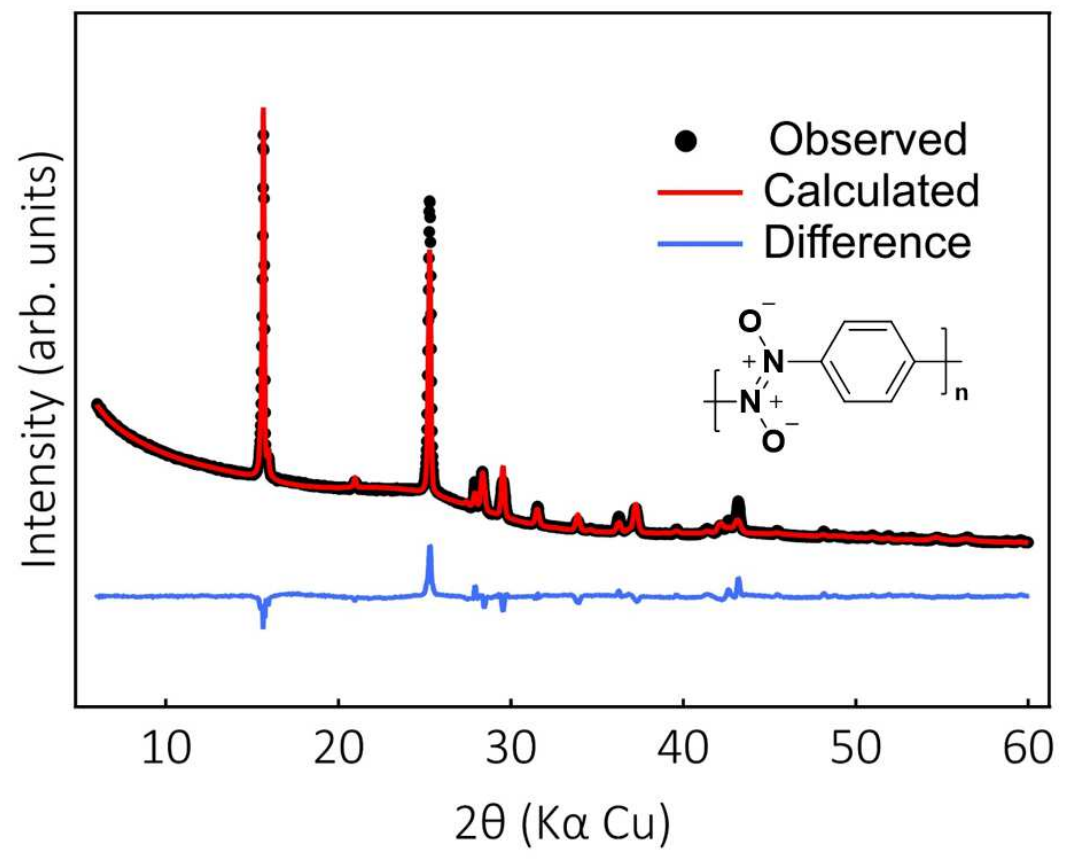

Supplementary Figure S19. Crystal structure analysis of PNND.

Experimental X-ray powder diffraction pattern (black dotted line) compared with the Rietveld-refined profile (red curve) and the difference (blue curve) for PNND. 

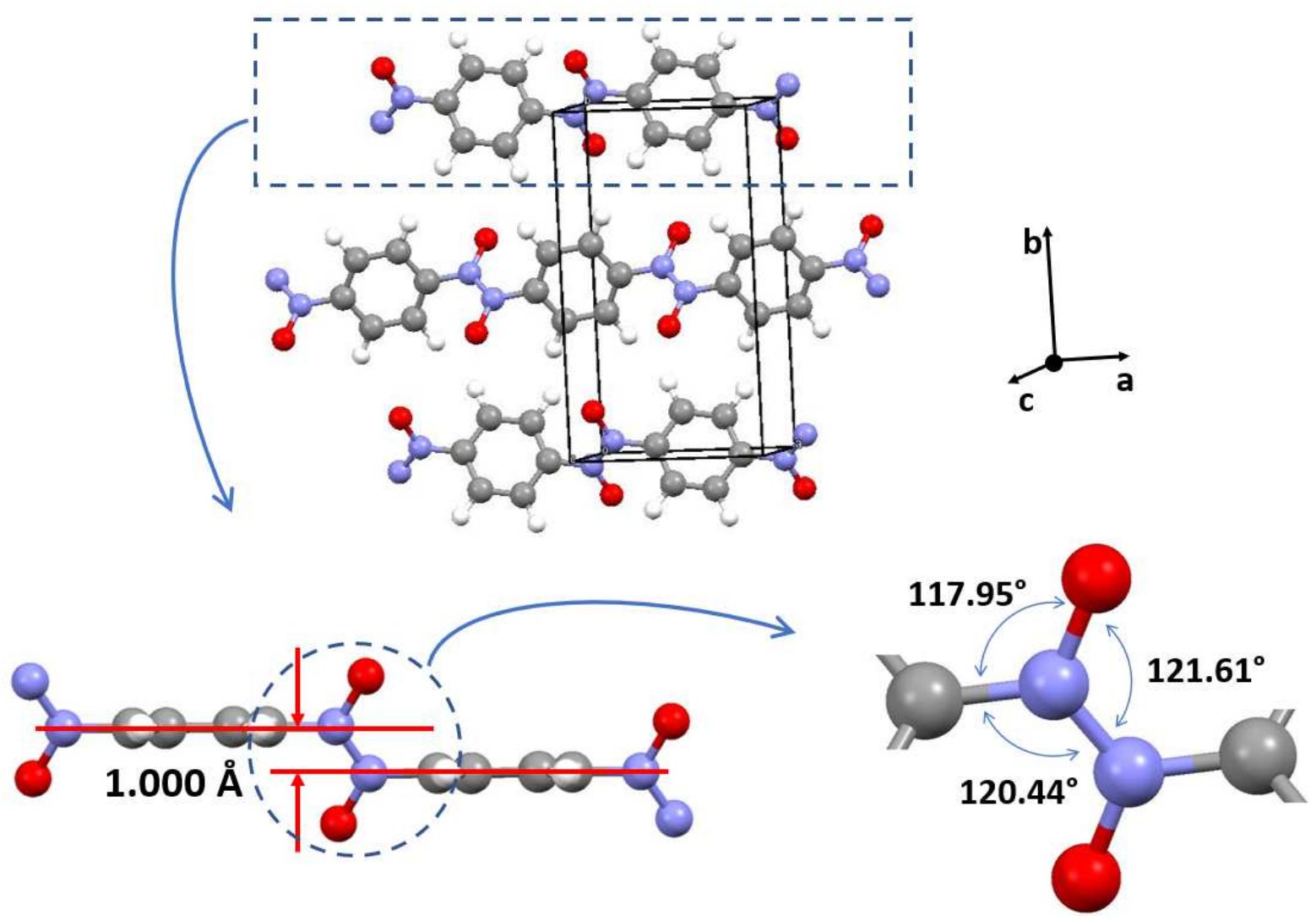

Supplementary Figure S20. Crystal structure of PNND polymer.

The crystal structure reveals that the polymer crystallizes in the monoclinic space group $P 2{ }_{1} / n\left(a=6.394(2) \AA, b=11.322(4) \AA, c=3.7072(1) \AA, b=92.923(6)^{\circ}\right.$, and $V=268.03(15) \AA^{3}$, Table S1).

Structure is similar to the reported CCDC structure with reference no. 2006601. Crystal structure shows that the compound polymerizes following the $E$ configuration along the $\mathrm{N}=\mathrm{N}$. Therefore, the PNND polymer is made up of linear chains, which go parallel along the crystallographic $a$-axis and stack along the $c$-axis. In the polymeric chain, the atoms do not lie on the same plane, but they are distributed on different planes defined by the aromatic ring and the $-\mathrm{ON}=$ NO- connection. The dihedral angle formed by the two planes leads to a stairlike structural motif in the polymeric chains. 
Supplementary Table S6. Solubility of electrode materials in 1M LiTFSI in EC/DMC electrolyte formulation.

\begin{tabular}{|c|c|c|}
\hline Entry & Composition & Solubility (mM) \\
\hline 1 & $\mathrm{Li}_{2}-\mathrm{BQDO}$ & 5.25 \\
\hline 2 & PNND e-oxidized $^{a}$ & 0.50 \\
\hline 3 & PNND chemically synthesized & 0.18 \\
\hline 4 & $\mathrm{Li}_{2}-\mathrm{DMGO}$ & 6.42 \\
\hline 5 & $\mathrm{Li}_{2}-\mathrm{DPGO}$ & 6.40 \\
\hline 6 & DPODO & 171.30 \\
\hline 7 & $\mathrm{Li}_{2}-\mathrm{PADO}$ & 4.21 \\
\hline 8 & $\mathrm{Li}_{4}-\mathrm{TMTO}$ & 1.77 \\
\hline
\end{tabular}

${ }^{a}$ Monomer molecular weight was used for molarity calculation. For calibration curve of chemically synthesized $P N N D, D M S O$ was used as a solvent. The analyzed sample was obtained by dissembling the cell with the $L i_{2}-B Q D O$ active material after $1^{\text {st }}$ charge, followed by dispersing the composite mixture in $1 M$ Li-TFSI in EC/DMC electrolyte. The resulting mixture was filtered through a $0.2 \mu$ syringe filter and was further diluted 10 times with DMSO.

For the neutral species (e.g. DPODO), high solubility was expected as the inter-molecular bonding in solid crystal are expected to be weak (no $\mathrm{H}$-bonding, presumably mainly through $\mathrm{VdW}$ bonding) thus highly soluble in polar solvents.

For the PNND polymer, low solubility was expected, and also confirmed by measurements, while also keeping in mind the particular dissolution mechanism and species - the dissolution of PNND takes place via reversible dissociation of azodioxy dimers, generation of the dinitroso benzene intermediate, and solubilization of the later (as confirmed by GPC measurements).

The solubility of $\mathrm{Li}_{2}-\mathrm{BQDO}$, and of other ionic lithiated oximate species, was in turn found higher than expected. However, this expectation was not based on quantifiable or established rules, but merely on general knowledge with organic battery materials, as well as literature survey. Typically, the ionic organic compounds have low solubility in battery electrolyte polar solvents, this being one strategy set forward for organic battery material stable cycling ${ }^{8}$. This can be assigned to relatively strong binding of Li-cations to the di-anionic organic centre (or framework) and the solubility thus dependent on the solvation strength (polarity, dielectric constant) of the used solvent.

In the studied series of compounds, a trend can be indeed identified, with the di-anionic species ( $\mathrm{Li}_{2}-\mathrm{BQDO}, \mathrm{Li}_{2}-\mathrm{DMGO}, \mathrm{Li}_{2}-\mathrm{DPGO}, \mathrm{Li}_{2}-\mathrm{PADO}$ ) having similar solubility in the range of 4-6 $\mathrm{mM}$; whereas the tetra-anionic $\mathrm{Li}_{4}$-TMTO has considerably lower solubility ( $2 \mathrm{mM}$ ) which could be explained by a stronger ionic binding to Li-ions, or dissolution via ion pairs to compensate for. 

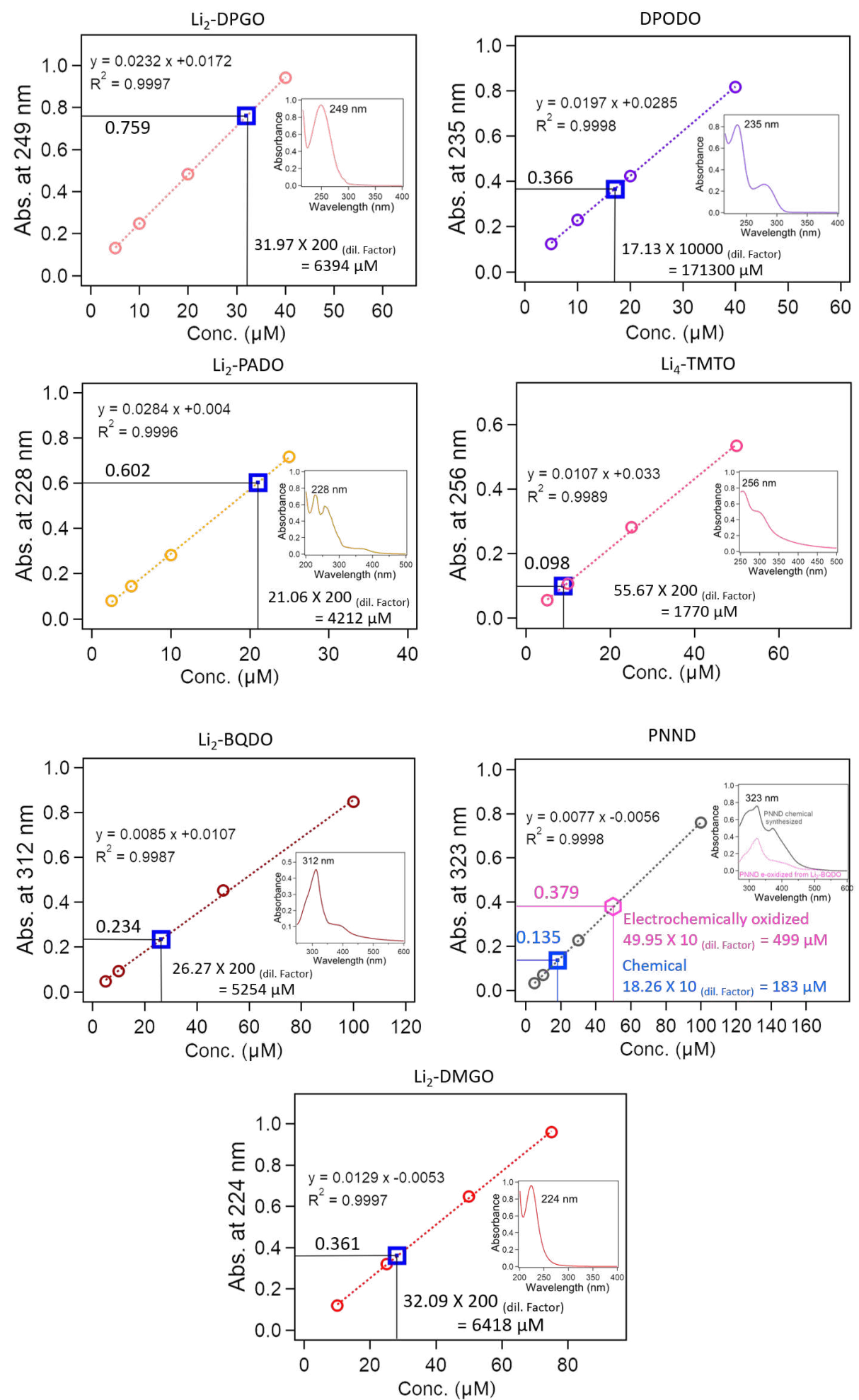

Supplementary Figure S21. UV-visible solubility calibration plots of the studied materials and their unknown diluted solutions in 1M Li-TFSI in EC/DMC electrolyte. Inset figure in each figure is respective UV-visible spectra. 
Except for PNND, stock solutions for all other electrode materials ( $\mathrm{Li}_{2}-\mathrm{BQDO}, \mathrm{Li}_{2}-\mathrm{DMGO}, \mathrm{Li}_{2}-$ DPGO, DPODO, $\mathrm{Li}_{2}-\mathrm{PADO}, \mathrm{Li}_{2}-\mathrm{TMTO}$ ) were made in $\mathrm{MeOH} / \mathrm{ACN}$ mixture, then dilutions were made with $A C N$ to prepare the calibration curves.

Saturated solutions of each material were prepared in electrolyte $1 \mathrm{M} \mathrm{Li-TFSI} \mathrm{in} \mathrm{EC/DMC}$ electrolyte. The resulting mixture was filtered through Millipore Millex-HV PVDF $0.2 \mu \mathrm{m}$ filters to remove the insolubilized solids. The filtrate was subsequently diluted in ACN/DMSO by a factor given indicated in every graph (Supplementary Figure 21). Four different standard solutions were prepared for each material to make the calibration curves.

The calibration curves were obtained by linear fit of maximum absorbance of the standard solutions vs concentration using the Beer-Lambert law: $A=\varepsilon \mid C$ ( $A$ : absorbance; $\varepsilon$ : molar extinction coefficient; I: length of the cell; C: electrode material concentration).

The solubility of the analysed materials was found to be relatively high in all cases (except PNND). These are following the trend (from highest to lowest soluble species): DPODO $>\mathrm{Li}_{2}-$ DMGO $>\mathrm{Li}_{2}$-DPGO $>\mathrm{Li}_{2}-\mathrm{BQDO}>\mathrm{Li}_{2}-$ PADO $>\mathrm{Li}_{4}-\mathrm{TMTO}>$ PNND e-oxidized. 

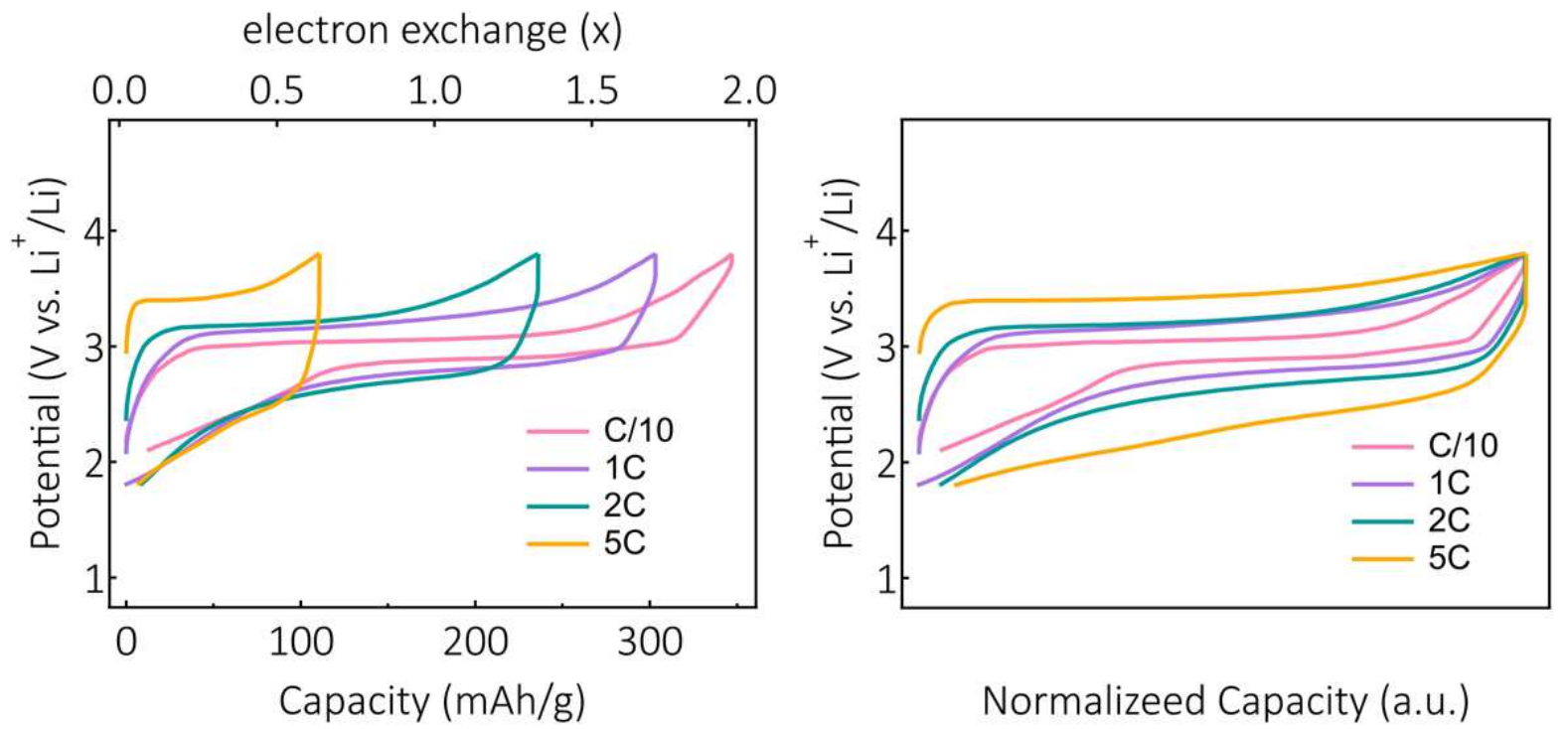

Supplementary Figure S22. Rate capability test of $\mathrm{Li}_{2}-\mathrm{BQDO}$.

The rate capability of the $\mathrm{Li}_{2}-\mathrm{BQDO}$ electrode (mass loading around $4 \mathrm{mg} / \mathrm{cm}^{2}$ ), measured at various $\mathrm{C}$-rates, in a half-cell configuration, and in $7 \mathrm{M} \mathrm{LiTFSI} \mathrm{in} \mathrm{EC/DMC} \mathrm{as} \mathrm{electrolyte.} \mathrm{The}$ electrode can still reach a high capacity, close to $300 \mathrm{mAh} / \mathrm{g}$, at a high 1C-rate (corresponding to 1 electron exchange in $30 \mathrm{~min}$ ).

From the Normalized capacity vs. Potential graph (right panel), an increase in the polarization with the increase of C-rate can be observed, attributed to a series of factors including working or Li-metal counter electrode polarization, as well as low ionic conductivity of the high concentration electrolyte. 


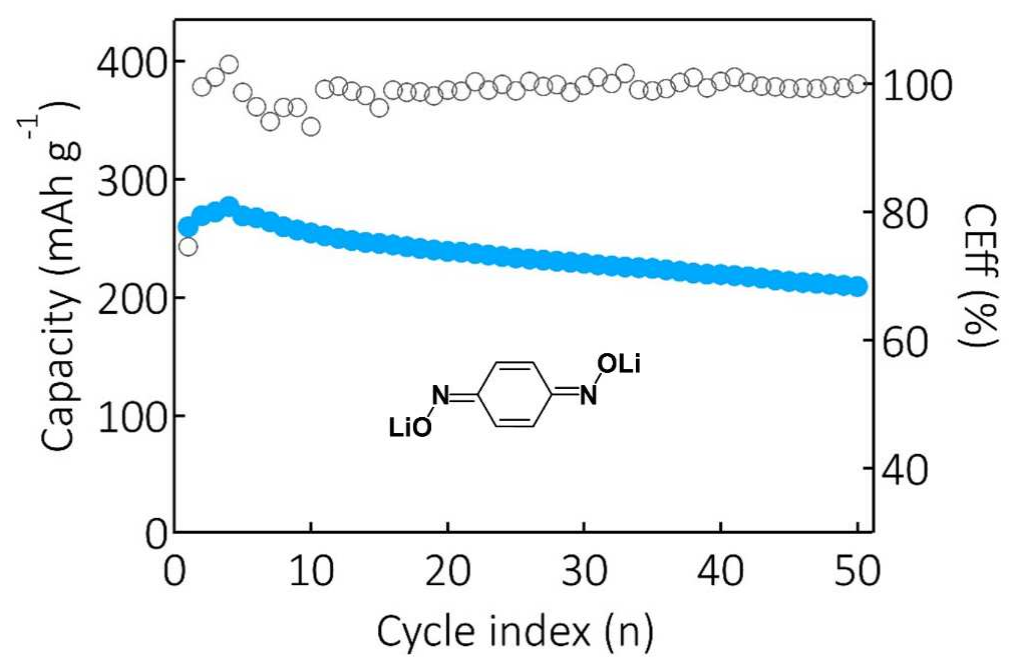

Supplementary Figure S23. The capacity retention and coulombic efficiency of $\mathrm{Li}_{2}-\mathrm{BQDO}$ cycled in a saturated LiTFSI in EC/DMC (1:1 vol\%) electrolyte. The cell was cycled a rate of $\mathrm{C} / 2$ (one electron exchange in $1 \mathrm{~h}$ ).

Compared to dilithium-1,4-benzenediolate, which can barely be cycled for a maximum of 5-10 cycles $^{9}$ (with more than $75 \%$ capacity loss) due to the very high solubility of $1,4-$ benzoquinone in electrolytes ${ }^{10}, \mathrm{Li}_{2}$-BQDO shows very stable cycling behavior with $75 \%$ capacity retention over 50 cycles. 


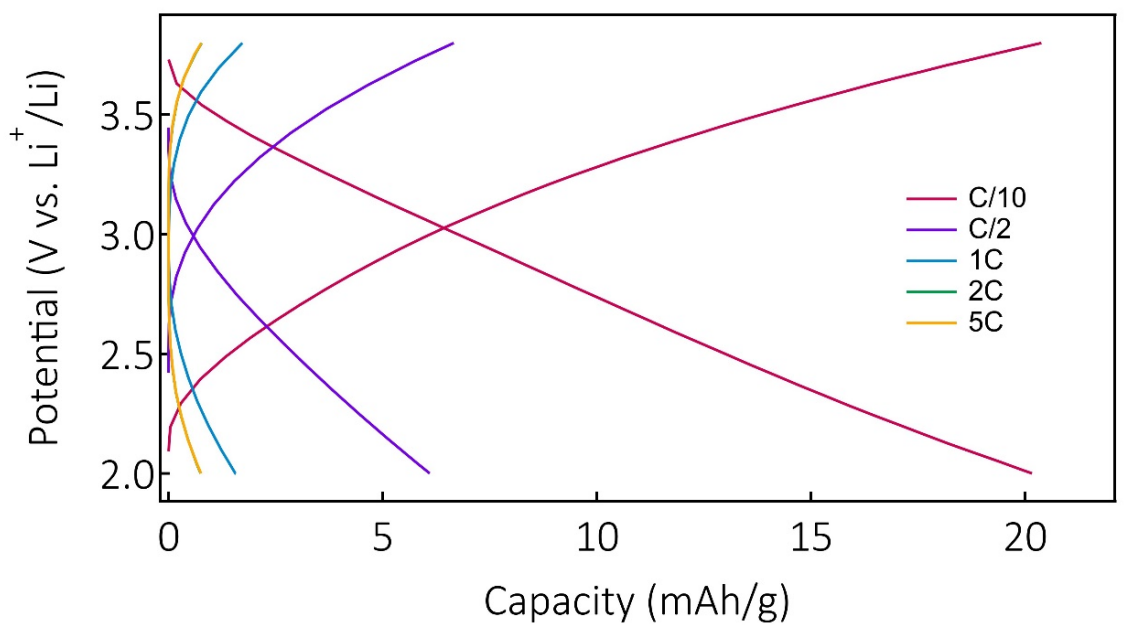

Supplementary Figure S24. Control experiment for the determination of the specific capacity contribution from Ketjen Black conductive carbon.

A pure Ketjen Black electrode was assembled in 2-electrode cell using Li-metal as counter/reference electrode and cycled in similar conditions as the studied active materials. For instance, the current corresponding to the C-rate applied in the graph above (for pure Ketjen Black electrode) is equal to the current densities applied to $\mathrm{Li}_{2}$-BQDO electrode (Fig. S22). 
$\mathrm{XLi}$ in the redox units

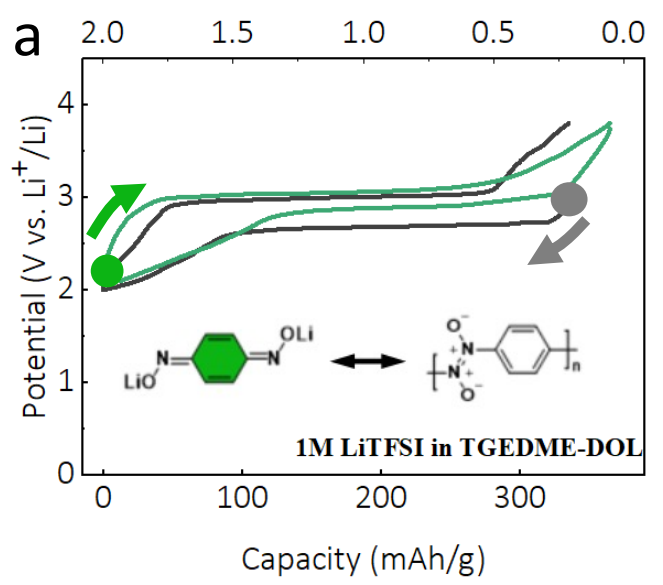

$\mathrm{XLi}$ in the redox units

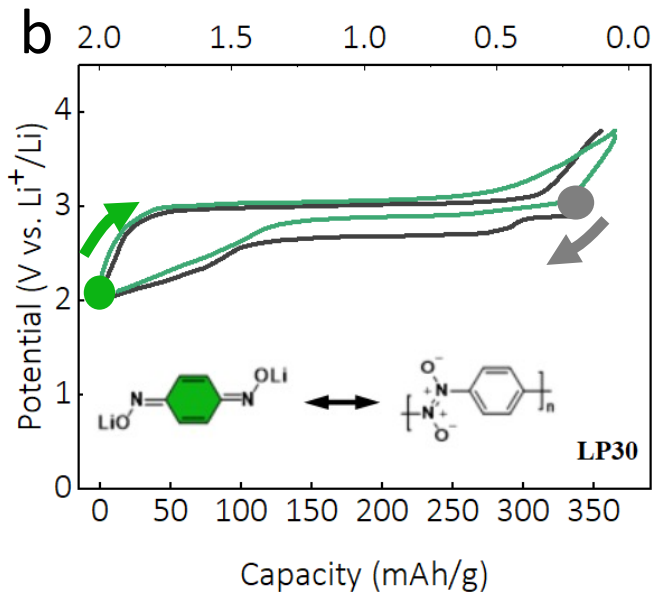

$\mathrm{XLi}$ in the redox units

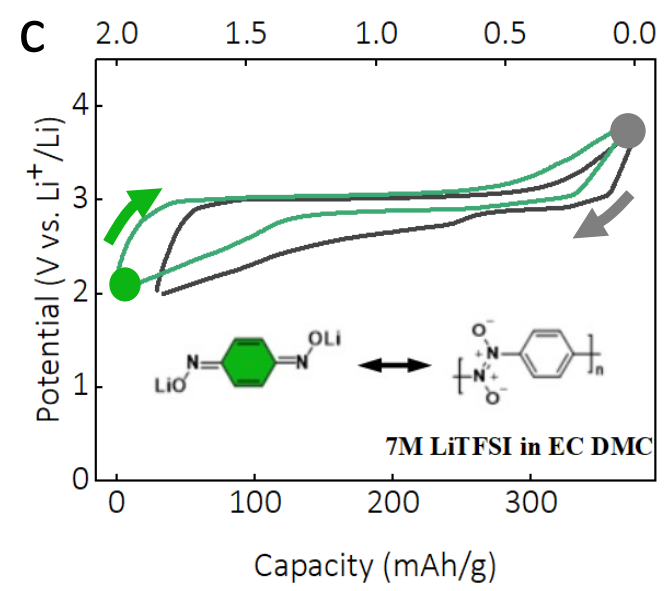

Supplementary Figure S25. Capacity-potential profile of chemically synthesized PNND and comparisons of cycling profiles of in different electrolytes.

$(a, b, c)$ Comparisons of cycling profiles of chemically prepared PNND and $\mathrm{Li}_{2}-\mathrm{BQDO}$. The electrodes, cell assembly and testing were performed in similar conditions with the exception of the used electrolyte.

(d) The top panel shows the charge-discharge profile of the PNND polymer prepared by chemical oxidation. The measurement was performed in a 2-electrode half-cell configuration using PNND as positive electrode material (electrode prepared with 50wt.\% PNND, 40wt.\% Ketjen black conductive carbon and 10\% PTFE binder), and Li-metal as reference/counter electrode; and cycled at a current density of $40 \mathrm{~mA} / \mathrm{g}$ (equivalent of $\mathrm{C} / 10$ rate).

Overall, the chemically prepared PNND and $\mathrm{Li}_{2}-\mathrm{BQDO}$ have similar galvanostatic cycling profiles, further corroborating the two-electron reversible redox mechanism between PNND (oxidized state) and $\mathrm{Li}_{2}-\mathrm{BQDO}$ (reduced state), and the reverse process. 
a<smiles>ON=C1C=CC(=NO[GeH3])C=C1</smiles><smiles>COc1ccc(OC)cc1</smiles>

$3.0 \mathrm{~V}$ vs. $\mathrm{Li}^{+} / \mathrm{Li}$, air-stable

$200 \mathrm{mV}$ raise

$2.8 \mathrm{~V}$ vs. $\mathrm{Li}^{+} / \mathrm{Li}$, air-sensitive

b

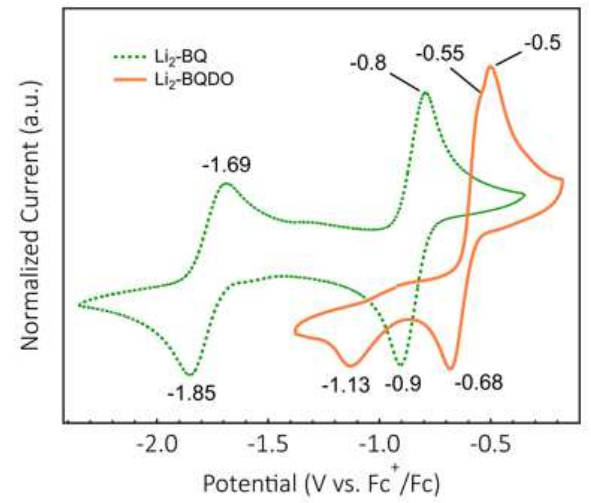

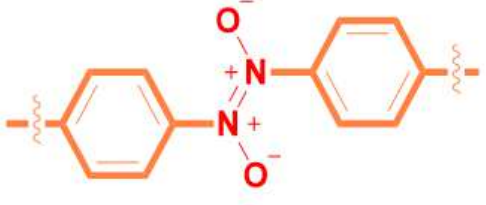

PNND

$$
\frac{-2 \mathrm{Li}^{+},-2 \mathrm{e}^{-}}{\stackrel{+2 \mathrm{Li}^{+},+2 \mathrm{e}^{-}}{\longrightarrow}}
$$

Polymeric insoluble oxidized phase

No solubility issue

Highly soluble oxidized phase

C

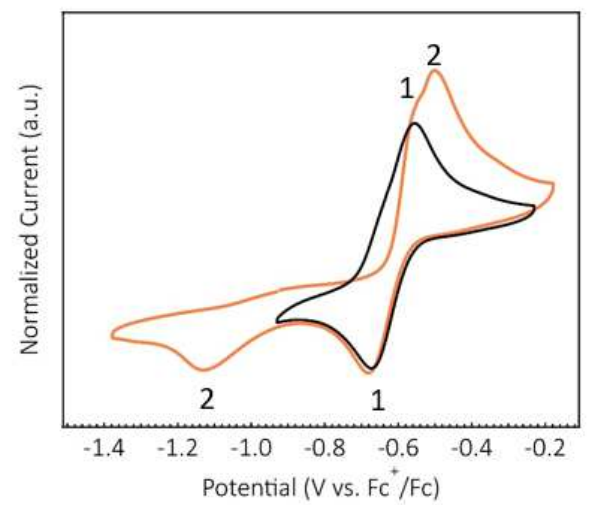

Supplementary Figure S26. Comparison of electrochemical properties for the main building units studied in this work: dilithium-1,4-benzenediolate ( $\left.\mathrm{Li}_{2}-\mathrm{BQ}\right)$ vs. dilithium $p$ Benzoquinone dioxime (Li2-BQDO).

a) The redox reaction of $\mathrm{Li}_{2}-\mathrm{BQDO}$ and of $\mathrm{Li}_{2}-\mathrm{BQ}$, highlighting the difference in the polymeric and single molecule oxidation reaction products. b) Cyclic voltammetry curve of $\mathrm{Li}_{2}-\mathrm{BQ} 100$ $\mathrm{mM}$ tetrabutylammonium perchlorate in DMSO) overlapped with CV of $\mathrm{Li}_{2}-\mathrm{BQDO}(100 \mathrm{mM}$ $\mathrm{LiCl}$ in $\mathrm{DMSO})$. The measured potentials were calibrated with a $\mathrm{Fc}^{+} / \mathrm{Fc}$ internal reference. The analysis shows higher redox potential of $\mathrm{Li}_{2}-\mathrm{BQDO}$ compared to $\mathrm{Li}_{2}-\mathrm{BQ}$, with two pairs of redox waves (anodic peaks: -1.13/-0.55, -0.68/-0.5 V for Li 2 -BQDO and -1.85/-1.69, -0.9 V/-0.8 V for $\mathrm{Li}_{2}-\mathrm{BQ}$ vs. $\mathrm{Fc}^{+} / \mathrm{Fc}$ ), indicating a two-electron stepwise process. c) Cyclic voltammetry curves of $\mathrm{Li}_{2}$-BQDO measured in two different potential windows: orange curve - full scan window, with the two cathodic processes observed, and narrow potential scan window (black curve) with only one pair of redox peaks noted. The redox pairs are noted with 1 and 2 and highlight the asymmetric anodic and cathodic reaction pathways of $\mathrm{Li}_{2}$-BQDO. 


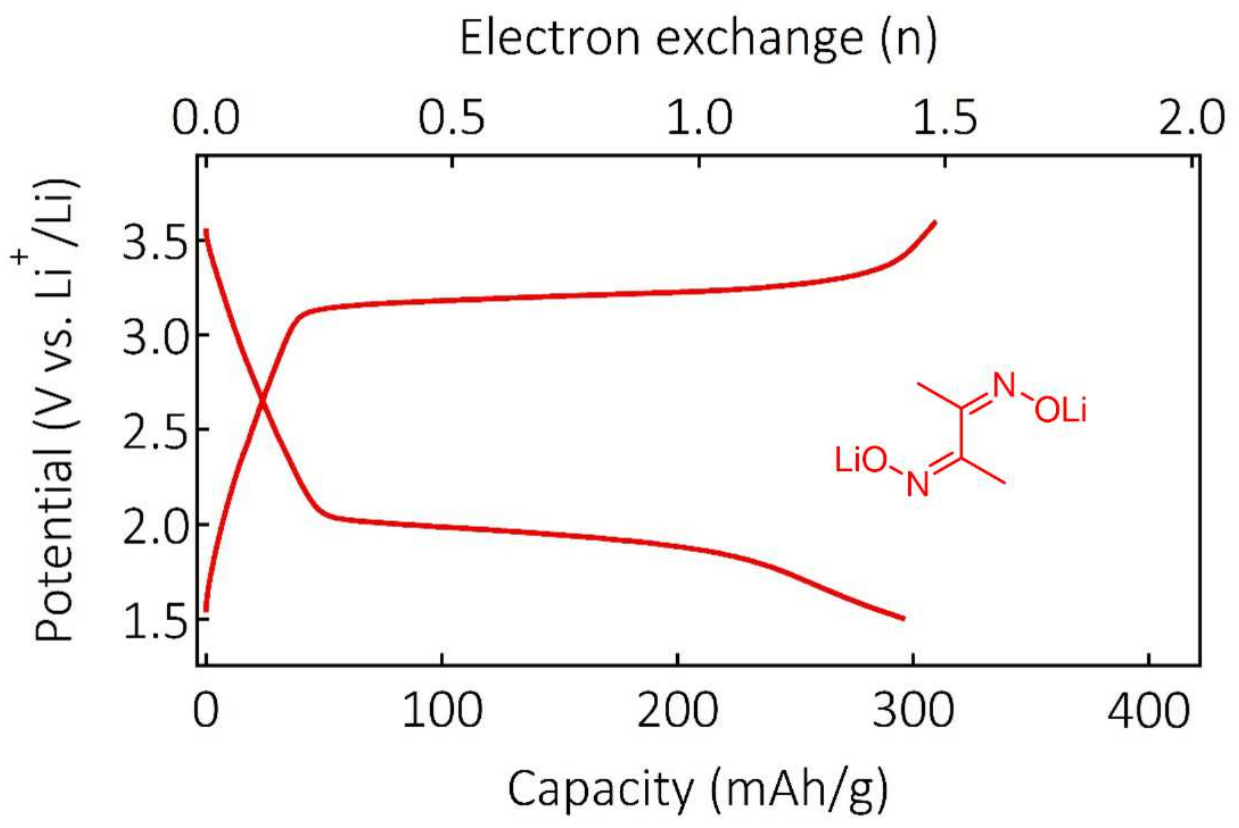

Supplementary Figure S27. Solid-state electrochemistry of dilithium-dimethylgyloxime ( $\mathbf{L i}_{2}$ DMGO).

Charge-discharge profile of $\mathrm{Li}_{2}$-DMGO electrode material as measured in a Lithium half-cell. The measurement was done with cycling rate of $\mathrm{C} / 10$ in a $5 \mathrm{M}$ LiTFSI in tetraglyme electrolyte, with an active material mass loading of $4 \mathrm{mg} / \mathrm{cm}^{2}$. The charge plateau is located at around 3.1V vs. $\mathrm{Li}^{+} / \mathrm{Li}$, while the discharge plateau is around $2.1 \mathrm{~V} \mathrm{vs}$. $\mathrm{Li}+/ \mathrm{Li}$, resulting in a large polarization of nearly $1 \mathrm{~V}$. The material utilisation is of 1.5 electron exchange (for a theoretical of two electrons), explained by the high solubility and elution from the electrode of both $\mathrm{Li}_{2}$ DMGO (Table S6) and its oxidized form (3,4-dimethyl-1,2,5-oxadiazole 2-oxide, that is a liquid) phases. 


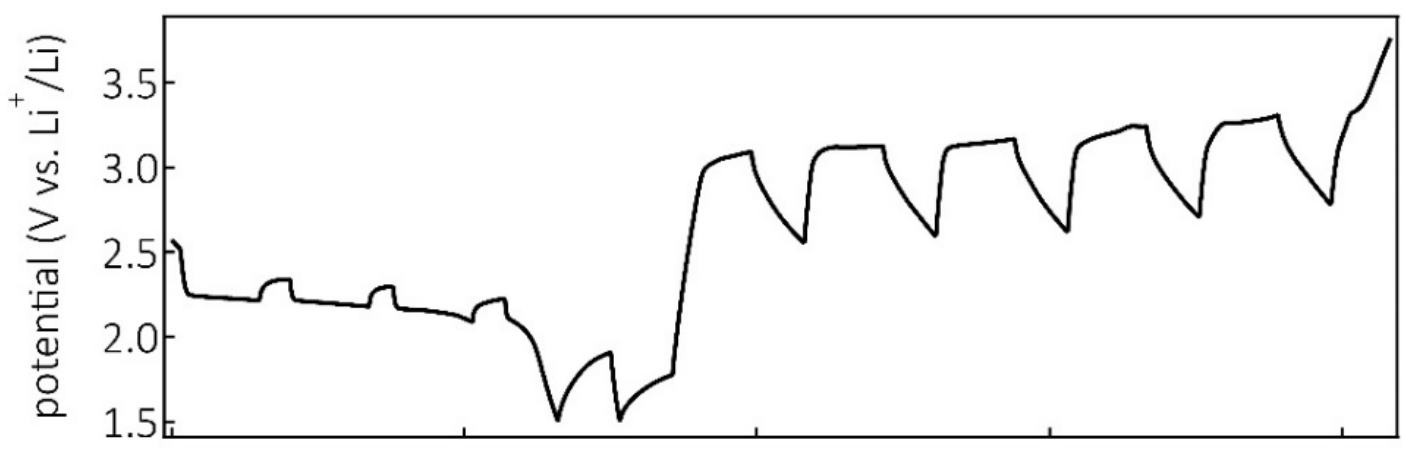

Time (t)

Supplementary Figure S28. Galvanostatic Intermittent Titration Technique (GITT) plot of the $\mathrm{Li}_{2}$-DPGO - DPODO redox as measured in a Li half cell configuration.

The test was performed with intermittent discharge/charge periods of 2 hours (rate of $C / 10$ ) followed by relaxation periods of 1 hour. The data shows that the main contribution to the polarization originates during the oxidation process ( $\mathrm{Li}_{2}$-DPGO $\rightarrow$ DPODO, over $500 \mathrm{mV}$ with the equilibrium not reached within one hour). Longer relaxation time have been also applied to allow the equilibrium to be reached, yet the solubility resulted in cells failure. The reduction process (DPODO $\rightarrow \mathrm{Li}_{2}-\mathrm{DPGO}$ ) only contributes by $\sim 150 \mathrm{mV}$ to the overall polarization, with the equilibrium reached within $1 \mathrm{~h}$ of relaxation. 


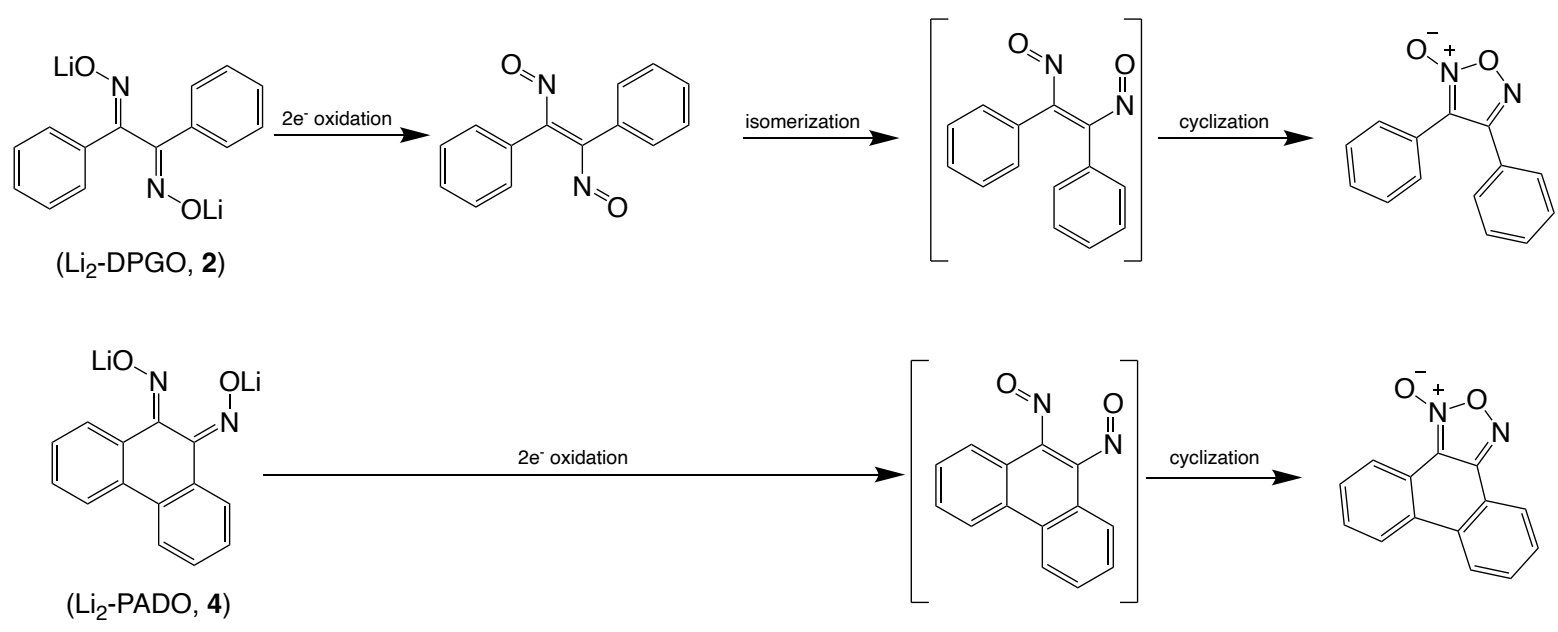

Supplementary Figure S29. Structural reorganization of $\mathrm{Li}_{2}-\mathrm{DPGO}$ and $\mathrm{Li}_{2}-\mathrm{PADO}$ during the oxidation process.

The aim of investigating the electrochemical performance of dilithium 9,10-phenanthrene dioximate $\left(\mathrm{Li}_{2}\right.$-PADO, 4$)$ was based on the assumption that the kinetically limiting step (and thus contributing to large polarization observed in galvanostatic cycling, Fig. 3 main text) is the $E-Z$ isomerization step of $\left(\mathrm{Li}_{2}-\mathrm{DPGO}, 2\right)$. However, high large hysteresis of $\sim 1 \mathrm{~V}$ was also observed for $\mathrm{Li}_{2}-\mathrm{PADO}$, indicating that the $E$ - $Z$ isomerization is not the main contributor to the cell polarization (Fig. 3c). The experimental results have thus been corroborated to calculations for the energy profile of the $\mathrm{Li}_{2}$-DPGO $\leftrightarrow$ DPODO conversion process (Fig. 3e, analysing both, linear and closed structure reaction pathways, and in both, gas and solid phases). 


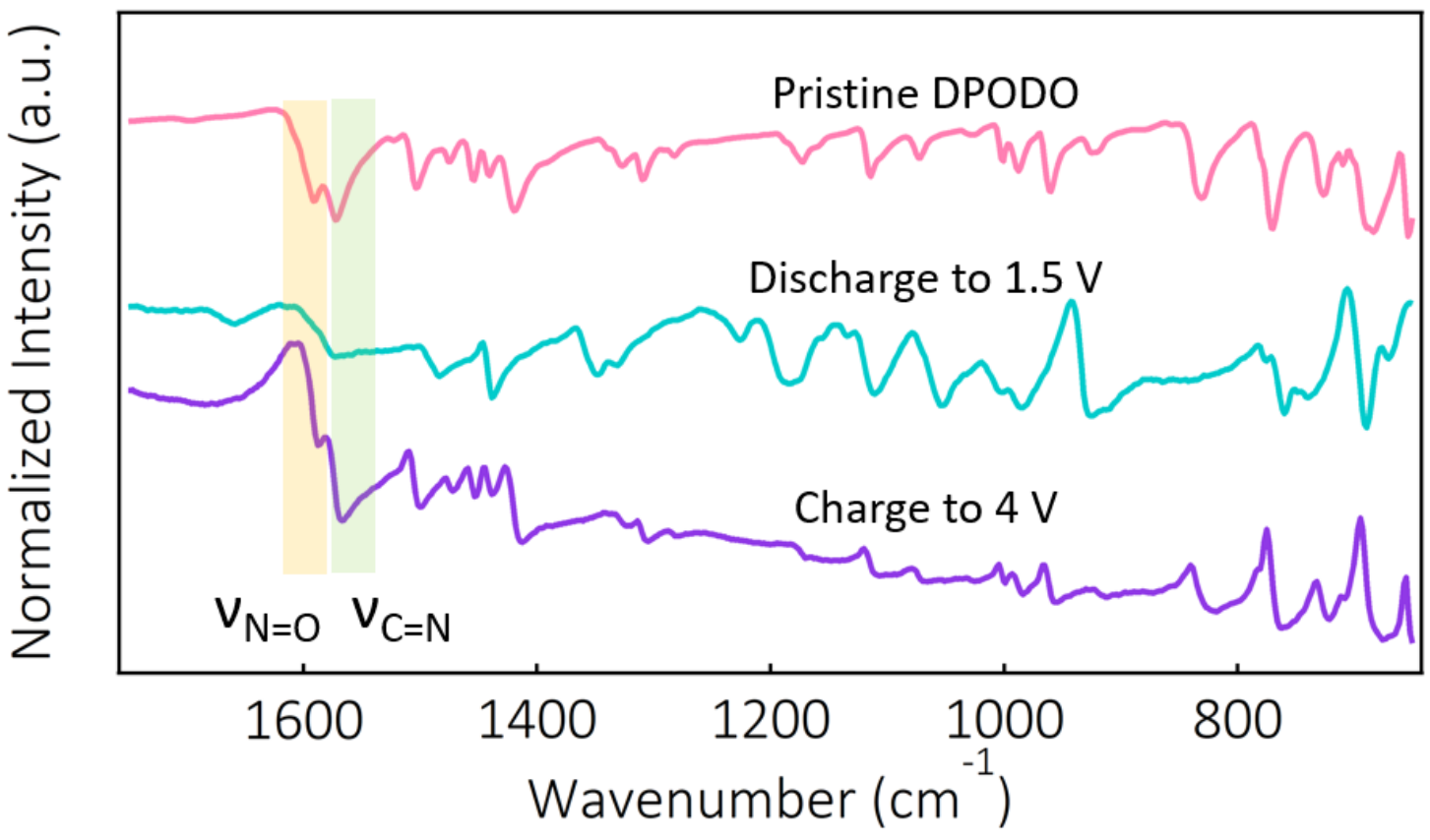

Supplementary Figure S30. Ex-situ FTIR survey of reversible electrochemical reaction between $\mathrm{Li}_{2}-\mathrm{DPGO}$ and DPODO in solid phase.

Ex-situ FTIR analysis was carried during the cycling of DPODO electrode (70\% active material and $30 \%$ conductive carbon). Three different state-of-charge states were analysed: pristine (oxidized DPODO form), two electrons reduced (DPODO $\rightarrow \mathrm{Li}_{2}$-DPGO, discharged to $1.5 \mathrm{~V} \mathrm{vs} \mathrm{Li}^{+} / \mathrm{Li}$ ) form, and one full redox cycle (Charge to $4 \mathrm{~V}$ vs $\mathrm{Li}^{+} / \mathrm{Li}, \mathrm{DPODO} \rightarrow \mathrm{Li}_{2}-\mathrm{DPGO} \rightarrow$ DPODO). The analysis shows excellent reversibility, given the identical spectra between the pristine and full-cycled data. The full-discharged spectrum was also found similar to the one of the as synthesized $\mathrm{Li}_{2}$-DPGO sample (Fig. S15), corroborating the electrochemical redox mechanism between DPODO and $\mathrm{Li}_{2}$-DPGO. 


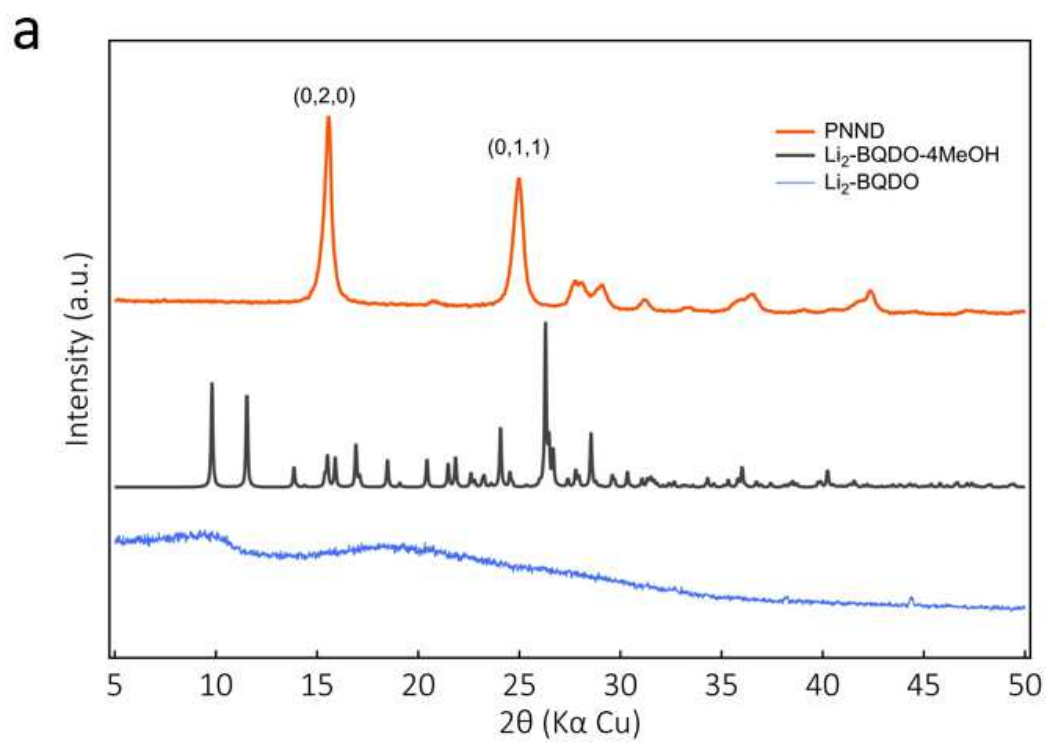

b

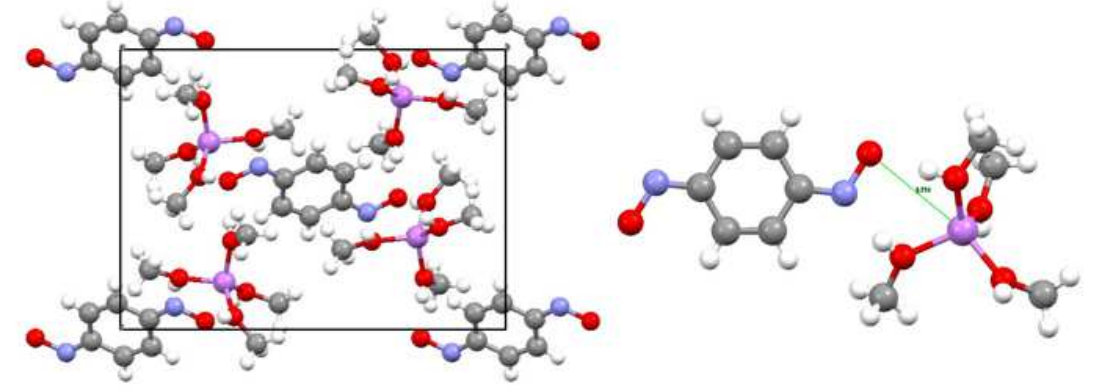

Supplementary Figure S31. PXRD data of $\mathrm{Li}_{2}-\mathrm{BQDO}$ and PNND phases and analysis of $\mathrm{Li}_{2}-$ BQDO-8MeOH crystal structure.

(a) PXRD of PNND polymer, of $\mathrm{Li}_{2}-\mathrm{BQDO}-8 \mathrm{MeOH}$ and of $\mathrm{Li}_{2}-\mathrm{BQDO}$ phases. The crystal structure of PNND polymer was solved from the PXRD data (Figs. S19 and S20) whereas the crystal structure of $\mathrm{Li}_{2}-\mathrm{BQDO}-8 \mathrm{MeOH}$ was obtained from single crystal data analysis.

An interesting feature worth to be noted is that $\mathrm{Li}_{2}-\mathrm{BQDO}-8 \mathrm{MeOH}$ is crystalline (black curve), whereas removing $\mathrm{MeOH}$ at $150{ }^{\circ} \mathrm{C}$ results in an amorphous $\mathrm{Li}_{2}-\mathrm{BQDO}$ phase (blue curve). The amorphous nature of the $\mathrm{Li}_{2}-\mathrm{BQDO}$ is preserved even during electrochemical charge-discharge cycles (Fig. 4, Main text), implying an exotic <amorphous small molecule salt $>$ to <crystalline polymer> cyclic electrochemical conversion.

(b) The single crystals of $\mathrm{Li}_{2}-\mathrm{BQDO}-8 \mathrm{MeOH}$ were obtained by slow diffusion of diethylether into a solution of $\mathrm{Li}_{2}-\mathrm{BQDO}$ in methanol at $-30{ }^{\circ} \mathrm{C}$ over a period of one month. The X-ray diffraction analysis shows that $\mathrm{Li}_{2}-\mathrm{BQDO}-8 \mathrm{MeOH}$ crystallizes in the monoclinic space group $\mathrm{P}$ $21 / \mathrm{n}\left(\mathrm{a}=7.0409(4) \AA, b=15.3351(10) \AA, c=11.1693(7) \AA, \beta=94.206(6)^{\circ}\right.$, and $V=1202.73$ $\AA$ 3. Each of the oximate function of the molecule binding two Li cations, which are solvated by four methanol molecules in a tetrahedral coordination. 
Supplementary Table S7. Crystal data and structure refinement for $\mathrm{Li}_{2}-\mathrm{BQDO}-8 \mathrm{MeOH}$ :

\begin{tabular}{|c|c|}
\hline Empirical formula & $\mathrm{C}_{14} \mathrm{H}_{36} \mathrm{Li}_{2} \mathrm{~N}_{2} \mathrm{O}_{10}$ \\
\hline Formula weight & 406.33 \\
\hline Temperature $(\mathrm{K})$ & $100(2)$ \\
\hline Wavelength $(\AA)$ & 1.54184 \\
\hline Crystal system & Monoclinic \\
\hline Space group & $P 21 / n$ \\
\hline \multirow[t]{6}{*}{ Unit cell dimensions $\left(\AA^{\circ}{ }^{\circ}\right)$} & $a=7.0409(4)$ \\
\hline & $b=15.3351(10)$ \\
\hline & $c=11.1693(7)$ \\
\hline & $a=90$ \\
\hline & $b=94.206(6)$ \\
\hline & $g=90$ \\
\hline Volume $\left(\AA^{3}\right)$ & $1202.73(13)$ \\
\hline Z & 2 \\
\hline Density (calculated) $\left(\mathrm{g} / \mathrm{cm}^{3}\right)$ & 1.122 \\
\hline Absorption coefficient $\left(\mathrm{mm}^{-1}\right)$ & 0.778 \\
\hline$F(000)$ & 440 \\
\hline Crystal size (mm3) & $0.495 \times 0.453 \times 0.329$ \\
\hline Theta range for data collection $\left({ }^{\circ}\right)$ & 6.935 to 67.096 \\
\hline Reflections collected & 4644 \\
\hline Independent reflections & $2109[R$ (int) $=0.0311]$ \\
\hline Completeness to $\mathrm{q}=66.489^{\circ}(\%)$ & 98.7 \\
\hline Absorption correction & Semi-empirical from equivalents \\
\hline Max. and min. transmission & 1.00000 and 0.86329 \\
\hline Refinement method & Full-matrix least-squares on F2 \\
\hline Data / restraints / parameters & $2109 / 4 / 143$ \\
\hline Goodness-of-fit on F2 & 1.083 \\
\hline Final $R$ indices $[I>2 s(I)]$ & $R_{1}=0.0402, w R_{2}=0.0992$ \\
\hline $\mathrm{R}$ indices (all data) & $R_{1}=0.0483, w R_{2}=0.1089$ \\
\hline $\operatorname{Dr}(\max , \min )(\mathrm{e} . \AA \AA-3)$ & $0.182,-0.232$ \\
\hline
\end{tabular}



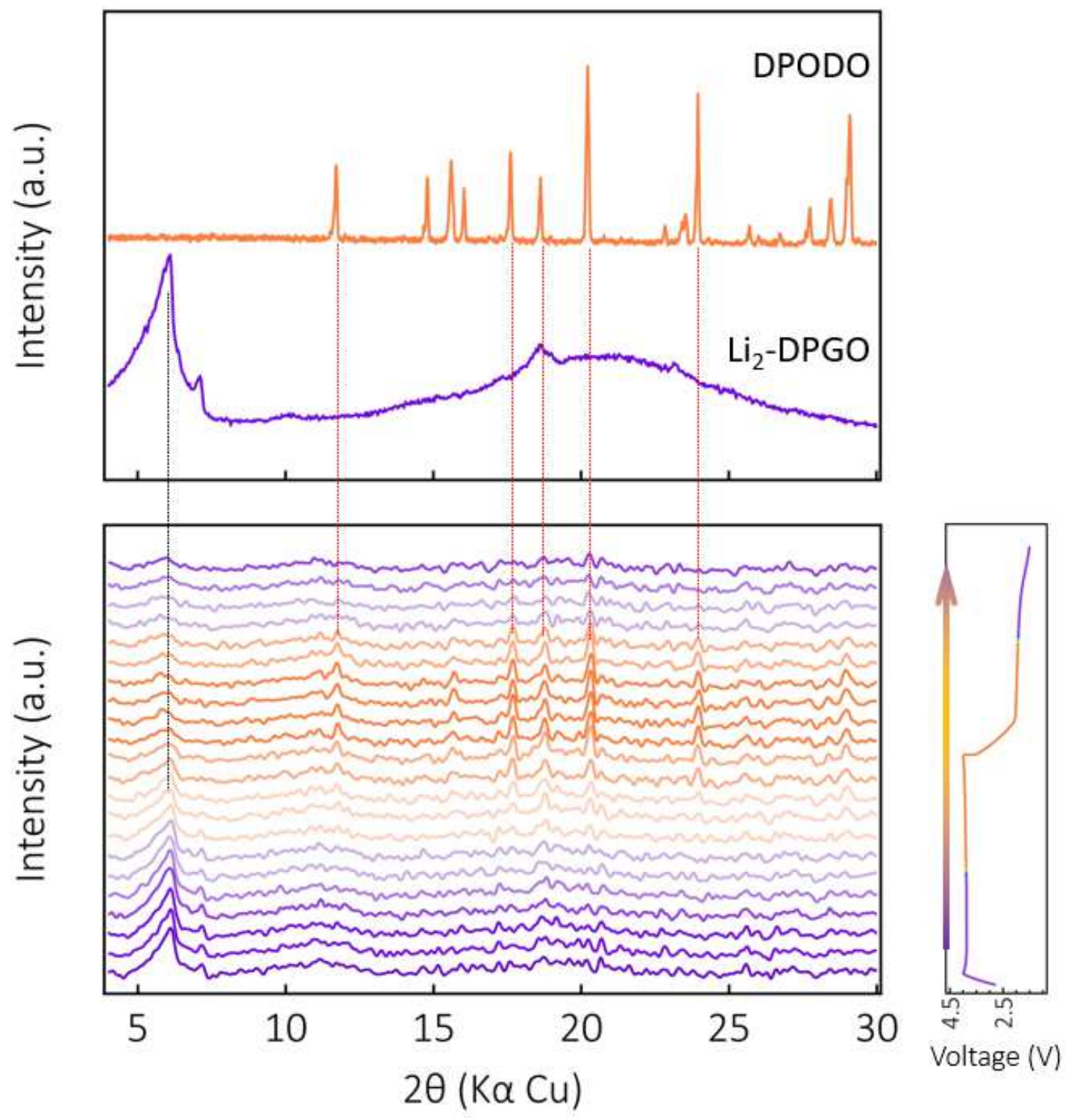

Supplementary Figure S32. In-situ XRD survey of $\mathrm{Li}_{2}$-DPGO during electrochemical cycling in solid phase.

The as synthesized $\mathrm{Li}_{2}$-DPGO is poorly crystalline (alike most of the Li-oximate derivatives disclosed in this work). Upon oxidation (charge), the $\mathrm{Li}_{2}$-DPGO phase gradually vanishes, simultaneous to the appearance of a new phase assigned to DPODO (matching the PXRD pattern of the chemically synthesized single crystal of DPODO ${ }^{11}$ ). The forward and reverse reactions thus proceed through a simultaneous two-electron, bi-phasic mechanism, corroborating the formation of furoxan ring after oxidation of $\mathrm{Li}_{2}-\mathrm{DPGO}$ (Fig. S29). Upon continuous cycling, the formed $\mathrm{Li}_{2}$-DPGO phase becomes less crystalline. 

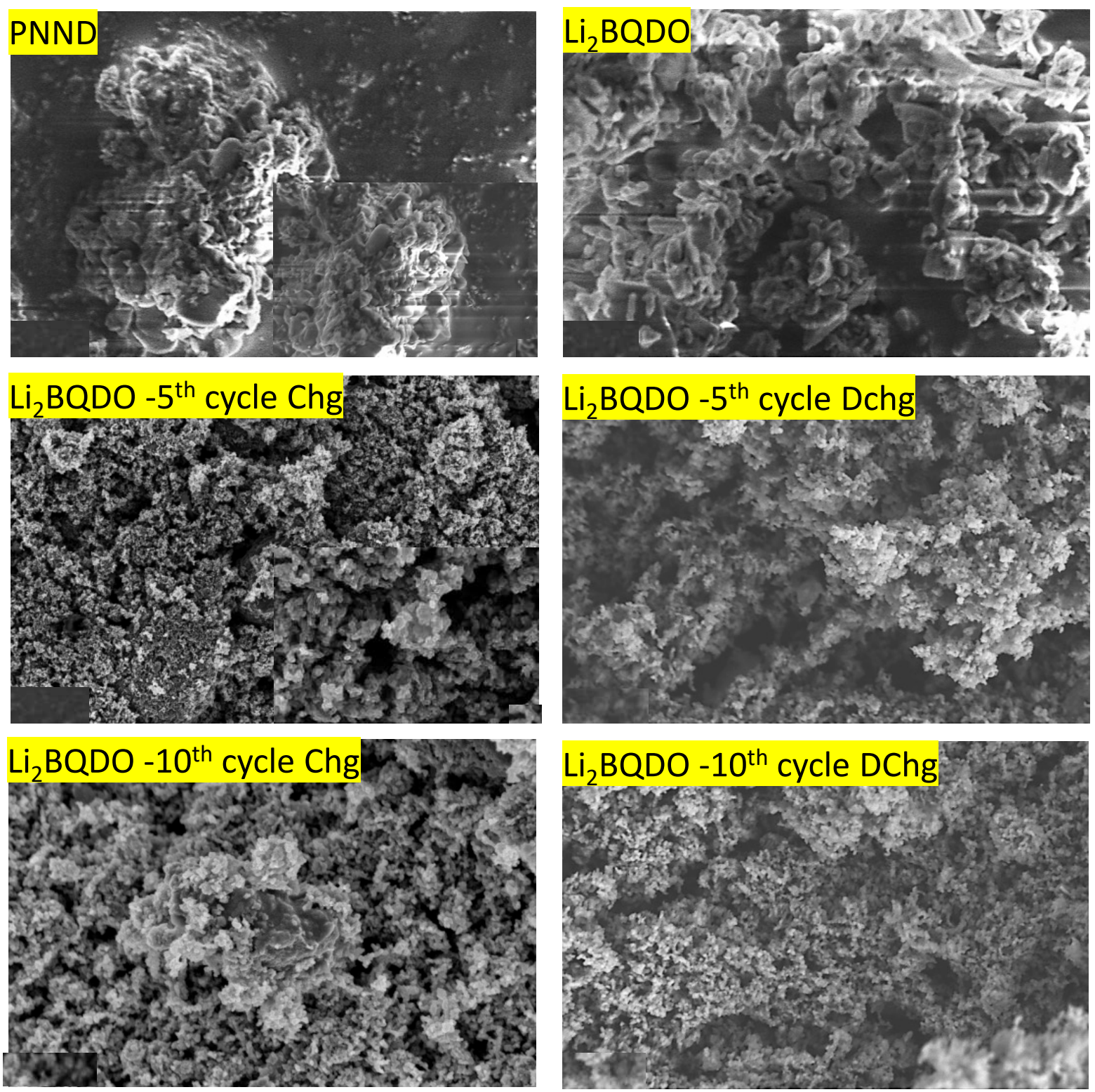

Supplementary Figure S33. SEM images of pristine PNND and $\mathrm{Li}_{2} \mathrm{BQDO}$ at different cycling sequences. 


\section{Gel Permeation Chromatography (GPC) analysis of soluble PNND species}

PNND (chemically synthesized)

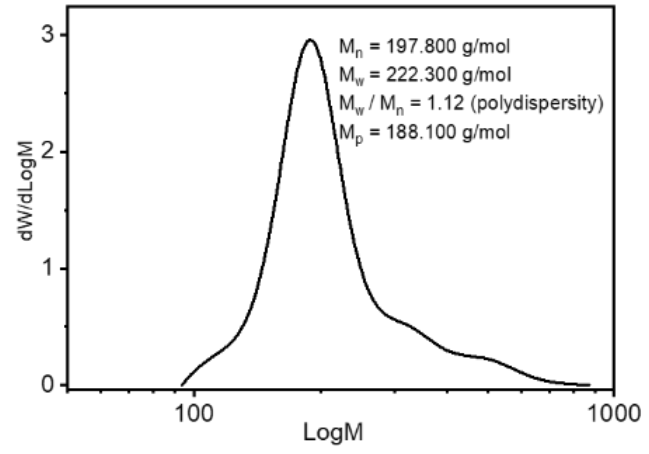

$\mathrm{Li}_{2} \mathrm{BQDO}$ after 10 cycle (charge)

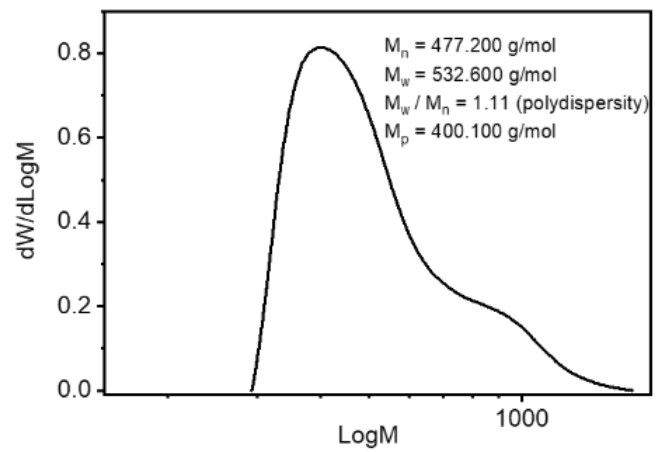

\section{GPC analysis of chemically synthesized PNND (left panel) and electrochemically synthesized PNND (right panel).}

The electrochemically synthesized PNND was prepared by galvanostatic cycling (10 full cycles) of $\mathrm{Li}_{2}$-BQDO, stopping the cycling in fully charged (oxidized) state, disassembling the cell, washing the positive electrode with DMC, followed by drying.

The PNND has low solubility in common solvents. Only partial solubility was observed in DMF and DMSO (Table S6) after long sonication. After this step, the DMF dispersion was centrifuged and the supernatant was filtered through a 0.2-micron filter before GPC analysis. The GPC was performed on an Agilent gel permeation chromatography (GPC) system equipped with an Agilent $1100 / 1200$ pump ( $25^{\circ} \mathrm{C}$; eluent: DMF, $2.5 \mathrm{mM}$ of $\mathrm{NH}_{4} \mathrm{PF}_{6}$; flow rate: $1 \mathrm{~mL} / \mathrm{min}$ ). The estimated $M$ are relative masses since these are determined with respect to polystyrene standards which have different hydrodynamic and elution volumes as compared to PNND, which is primarily composed of monomers and small-chain oligomers.

The analysis shows that the soluble species are of low $M$, in the range of the molecular weight of the 1,4-dinitrosobenzene monomer $(136,11 \mathrm{~g} / \mathrm{mol})$, with a series of peaks with lower intensity and corresponding to integer multiples of the main peak $\mathrm{Mw}$. The low polydispersity $(1.11-1.12)$ for a potentially non-controlled polymerization process is also an indication that the species resulting from the solubilization of PNND are monomers or few units (2-5) oligomers.

The solubilization via depolymerization can be explained by the low activation energy for the dissociation of azodoxy dimers $(20-30 \mathrm{kcal} / \mathrm{mol})$, implying that this covalent bonding can be broken - in this case, under the influence of polar solvents. This is also supported by previous findings wherein mono-nitroso compounds have been observed to behave differently in dissolved and solid phases: faintly yellow in the solid state, bright green in solutions ${ }^{12}$. This was attributed to the reversible dimerization of nitroso compounds with most nitrosoarenes thus existing as (dissolved) monomers in solution, and dimeric in the solid state, and at room temperature. 


\section{DFT Calculation Section.}

\section{The case study of $\mathrm{Li}_{2}$-DPGO $\rightleftarrows$ DPODO conversion.}

\section{Computational setup.}

DFT was performed for both gas- and solid-phase systems. The gas-phase simulations were performed with the GAMESS package ${ }^{13}$. Total energies for each of the oxidation states, $E_{\text {tot }}$ were computed for the relaxed structures using the Minessota M11/M15 exchangecorrelation functionals ${ }^{14}$ and the $6-31 G^{* *}$ Pople basis set. Solid-state calculations were performed using SIESTA ${ }^{15}$ which uses norm-conserving pseudopotentials and LCAO representation of the wavefunction. The exchange correlation functional used was PBE for solid (PBEsol $\left.{ }^{16}\right)$; this choice being known to produce reliable geometric structures for molecular crystals bulk states ${ }^{17}$. In order to take into account the van der Waals interaction, we applied the Grimme's corrections to PBEsol ${ }^{18}$.

\section{Geometric models.}

Gas phase calculations - were performed for two structures: closed and linear one (Figure S34). In all cases, the results are reported after performing a structural relaxation up to a gradient of $0.01 \mathrm{eV} / \mathrm{A}$.
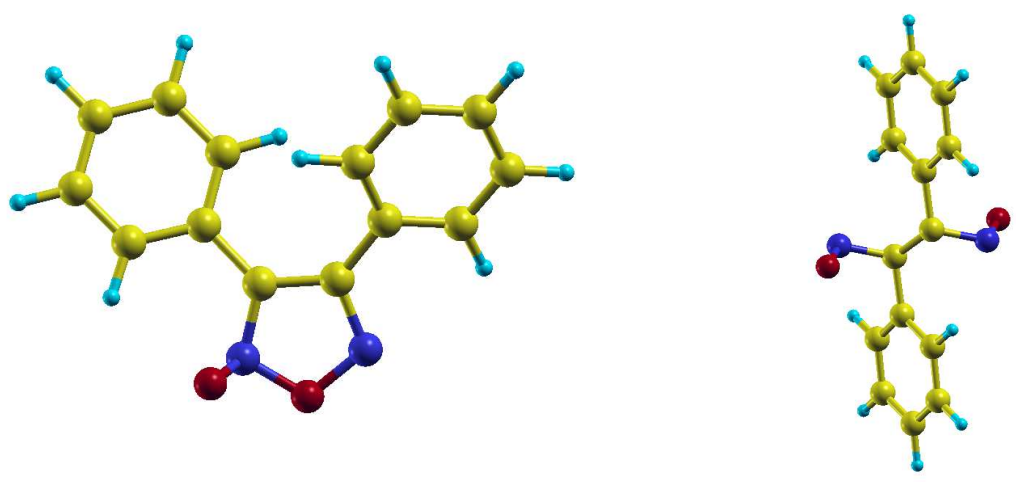

Supplementary Figure S34: Ball-and-stick representation for closed (left) and linear (right) structures of DPODO and DPGO2- in gas phase.

For the solid-phase calculations we start out investigations for two types of packing (i.e. for closed and linear structures, respectively). We used two types of computational models: in the first one, the structural relaxation is applied only to atoms in the cell (experimental geometry of the cell is preserved); second, the structural relaxation of the atomic positions and cell parameters is performed. For all models the structural relaxation was conducted to attain a gradient of less than $0.05 \mathrm{ev} / \AA ̊ \AA$. . Results for the relaxed cells are given in Table S7. 
Supplementary Table S8: Experimental (bold) and calculated values for the crystal structures for closed (Row 1) and for the linear structures (Row 2).

\begin{tabular}{lllllll}
\hline & $\boldsymbol{a}[\AA \AA$ & $b[\AA ̊$ & $\boldsymbol{C}[\AA ̊$ & Alpha $\left({ }^{\circ}\right)$ & Beta $\left({ }^{\circ}\right)$ & Gamma $\left({ }^{\circ}\right)$ \\
\hline closed & $15.15 / 14.28$ & $12.26 / 12.25$ & $12.66 / 11.64$ & $90 / 89.95$ & $94.36 / 101.05$ & $90 / 89.97$ \\
\hline linear & $11.42 / 11.95$ & $20.01 / 20.43$ & $11.70 / 8.90$ & $90 / 90$ & $114.71 / 115.01$ & $90 / 90$ \\
& & & & & & \\
\hline
\end{tabular}

To investigate the insertion of $\mathrm{Li}$ in the structures we used supercell models with $2 \times 2 \times 2$ unit cells, allowing more freedom to Li-ions (i.e. with respect to periodic boundary conditions) for both close/linear structures.

We calculated three models corresponding to a gradual insertion of $\mathrm{Li}$-ions into the organic bulk: no Li atoms included, 50\% of the maximum amount of Li atoms (i.e. 8 atoms in each super-cell i.e. a single $\mathrm{Li}$ atoms per molecule) and 100\% of $\mathrm{Li}$ atoms (i.e. $16 \mathrm{Li}$ atoms per supercell i.e. $2 \mathrm{Li}$ atoms per molecule). The initial guess for the positions of $\mathrm{Li}$ atoms was produced by generating random positions at distances between 2.8 and $2.5 \AA$ around the oxygen atoms, followed by full relaxation up to $0.05 \mathrm{ev} / \AA$. . As an example, the relaxed structures for $16 \mathrm{Li}$ atoms in the two types of supercells in given in Figure S35.

The effect of $\mathrm{Li}$ insertion (i.e. 100\% of Li atoms in the structure, that is 16 atoms/supercell) upon the cell parameters is presented in Table S8. As a qualitative comment we note that the effect of $\mathrm{Li}$ insertion upon the volume change of the unit cells is opposite for the two structures: for closed structure the volume is increasing in presence of $\mathrm{Li}$, while for the linear one the volume is decreasing.

Supplementary Table S9: Effect of the Li insertion upon the structural parameters of the crystals: calculated values for the crystal structure parameters in presence of $L i$ atoms for closed structure (Row 1, volume $=2050.03 \AA^{3}$ compared to $2344.65 \AA^{3}$ without Li) and for the linear one (Row 2, volume $=2014.04 \AA^{3}$ compared to $2427.212 \AA^{3}$ without Li). For comparison, refer to the values in Table S7 for the structures without $L i$.

\begin{tabular}{|c|c|c|c|c|c|c|}
\hline & $a[\AA ̊]$ & $b[\AA ̊]$ & $C[\AA ̊]$ & Alpha $\left({ }^{\circ}\right)$ & Beta $\left({ }^{\circ}\right)$ & Gamma $\left({ }^{\circ}\right)$ \\
\hline closed & 15.21 & 10.53 & 12.86 & 87.16 & 84.76 & 89.38 \\
\hline linear & 10.25 & 17.25 & 11.78 & 90.52 & 104.93 & 89.52 \\
\hline
\end{tabular}


a

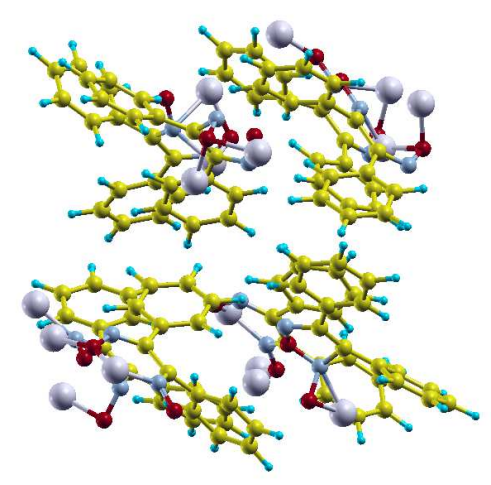

C

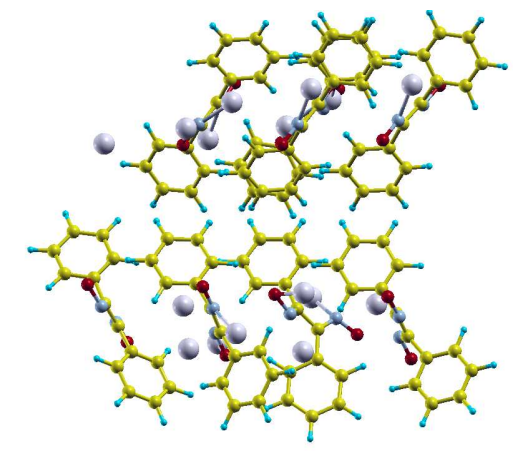

$\mathrm{b}$

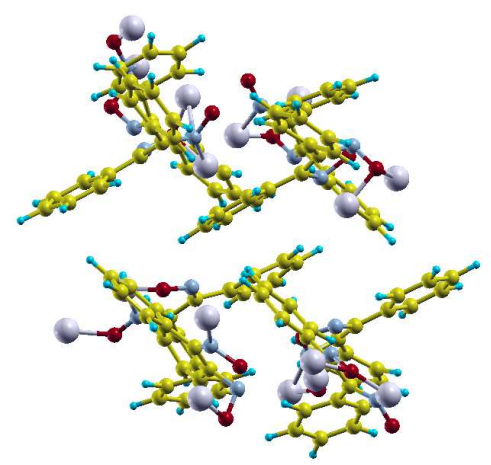

d

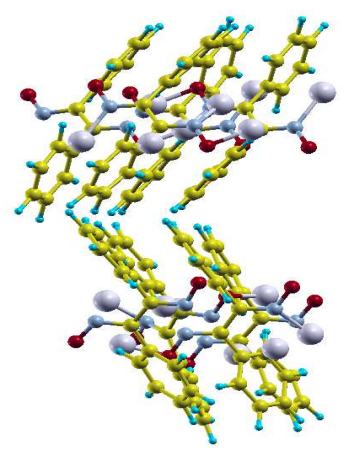

Supplementary Figure S35: Representation of the supercells used in calculations for closed structure ( $a$ and $b$ ) and for the linear one ( $c$ and d). Maximum number of Li atoms (i.e. 16) - represented with large gray balls - is included in each structure.

\section{Results and Discussions.}

\subsection{Geometric structures.}

The statistical distribution of selected bond lengths in various states was monitored by using histograms of interatomic pair distances. Since the system is periodic, not all the pairs are properly considered (i.e. the bonds at the border of the periodically repeated cell); the analysis on larger super-cells was thus performed, and then re-normalizing the total number to the cell used in calculations. Non-integer numbers in the histogram indicate atoms that are not properly taken into account due to periodic boundary conditions.

- $\rightarrow$ Li-O and Li-N pairs: A total of 100 steps between $1 \AA$ and $5 \AA$ was used to build the histograms, with the results for closed and linear structures being given in Figures S36 and S37, respectively. It can be noticed that for closed structure (Figure S36), Li atoms have the tendency to coordinate $\mathrm{O}$ atoms with a majority of $\mathrm{Li}-\mathrm{O}$ at a distance of about $1.9-2.0 \AA$. For the linear structure (Figure S37), Li atoms are coordinated equally to $\mathrm{O}$ and $\mathrm{N}$ atoms, with a slightly higher number of Li-N pairs (around 40), at distances equal to 2.1 and $2.3 \AA$, respectively, and similar number for Li-O pairs at distances of around $1.8-1.9 \AA$. 

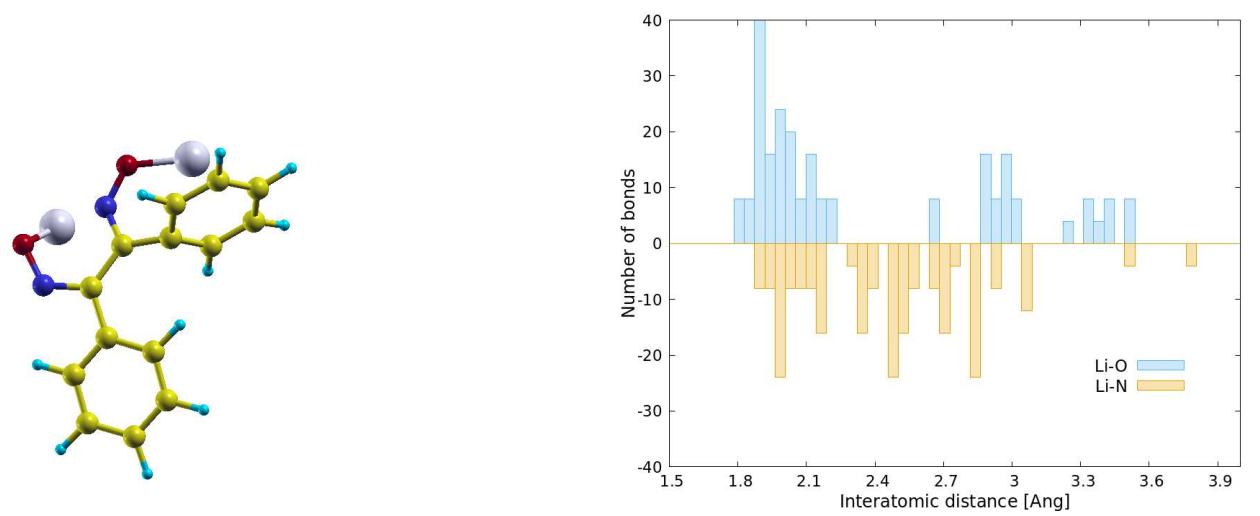

Supplementary Figure S36: Geometric analysis for closed structure. Left: geometric structure of a selected molecular unit in the bulk after structural relaxation in the presence of Li atoms (gray balls) - showing that the closed structure is not preserved in presence of the two Li atoms (i.e. the $\mathrm{N}-\mathrm{O}$ bond is broken). Right: histogram count of the Li-O (blue) and Li-N (yellow) distances in presence of Li atoms. While for the Li$\mathbf{N}$ we note a more disperse distribution of the bonds, the values of $\mathrm{Li}-\mathrm{O}$ are grouped around value $1.9 \AA$ A. Large distances (e.g. over $2.5 \AA ̊$ ) are purely geometric results, no chemical interactions can be present.
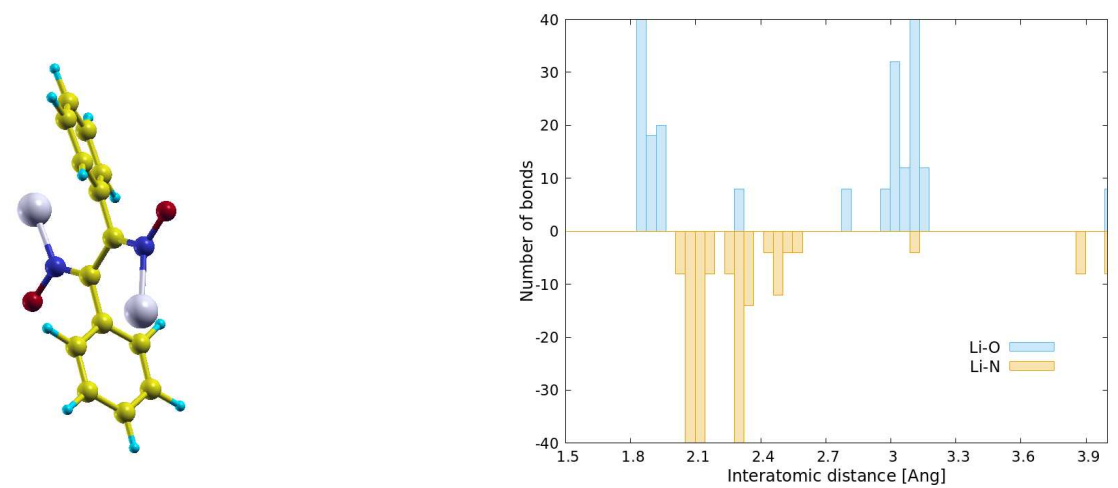

Supplementary Figure S37: Geometric analysis for linear structure. Left: geometric structure of a molecule in the bulk after structural relaxation in presence of Li (gray atoms). Right: histogram count the Li-O (blue) and Li-N distances (yellow), respectively, in presence of Li atoms; the distances are grouped around $1.9 \AA$ (for Li-O), and 2.1 - 2.3 $\AA$ (for Li-N). 
- $\rightarrow \mathbf{C}=\mathbf{N}$ bond: To investigate the $\mathrm{C}=\mathrm{N}$ bond evolution upon redox conversion, a smaller step in the histogram ( $0.002 \AA$ ) was used, since the $\mathrm{C}=\mathrm{N}$ is a significantly stronger bond. The results for the two structures and for the three Li concentrations (i.e. $0 \%, 50 \%$ and $100 \%$ ) are presented in Figure S38. In the absence of $\mathrm{Li}$ (no distortions of the structure) the number of corresponding $\mathrm{C}=\mathrm{N}$ bonds in the histogram is obtained. For the linear structure (top), in absence of $\mathrm{Li}$, sixteen $\mathrm{C}=\mathrm{N}$ bonds with lengths around $1.344 \AA$ (i.e. all bonds are equal in the absence of $\mathrm{Li}$ ) are counted. For the closed structure (bottom), two groups of eight $\mathrm{C}=\mathrm{N}$ bonds, with lengths around $1.342 \AA$ and $1.352 \AA$, respectively, are noted - corresponding to the two types of $\mathrm{C}=\mathrm{N}$ bonds in the closed structure.

The presence of $50 \%$ of all $\mathrm{Li}$ atoms leads to a random distribution of the $\mathrm{C}=\mathrm{N}$ bonds for both structures. Indeed, the orange histogram indicates an almost uniform distribution of the bonds, which is the indication that no dominant structure is present in the two structures (linear or closed one).

For the fully saturated structure $(100 \% \mathrm{Li})$, we note that in the closed structure the presence of a first $\mathrm{Li}$ atom has the effect that all $\mathrm{C}-\mathrm{N}$ distances tend to increase: all values are superior to $1.34 \AA$ (i.e. the bond length in absence of $\mathrm{Li}$ ), with about ten bonds counted for $1.36 \AA$. For the $\mathrm{C}=\mathrm{N}$ bond, this represents a weakening of the chemical bond in presence of $\mathrm{Li}$ atoms (longer distance, weaker bond). For the linear structure the effect is opposite, with many bonds centred around $1.33 \AA$ (inferior to $1.345 \AA$ in absence of Li). This indicates stronger bonding in presence of $\mathrm{Li}$ for the linear structure.

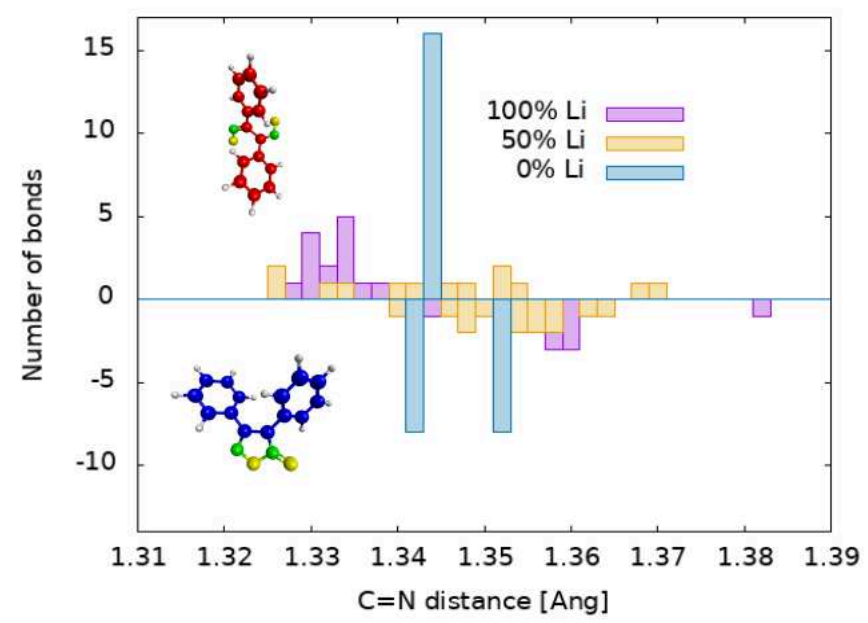

Supplementary Figure S38: Histogram of the C-N distances (nitrogen represented with green in the inset of the pictures, carbon with blue) as function of Li concentration for the two structural models presented in Figure S34. Top values- linear structure; bottom values - closed structure. It can be noted that presence of Li (yellow and purple boxes) has an opposite effect on the $\mathrm{C}-\mathrm{N}$ bond length in the two structures: smaller (1.333 $\AA$ ) for linear configuration, and larger (1.360 Å) for the closed configuration. 


\section{Summary of the geometric properties analysis:}

The impact of $\mathrm{Li}$ atoms on the molecular configuration for the two structures in solid phase (i.e. closed and linear) has an opposite effect, as follows:

- The volume of the elementary cell: linear structure has a lower volume of the unit cell, while the closed one has a larger volume of the unit cell, upon the insertion of Li.

- Strength of $C=N$ bond: in the linear structure a shortening of the bond is brought by the presence of $\mathrm{Li}$, while for the closed configuration, the bonds are longer (i.e. weaker).

- To note that the weakening of the $\mathrm{C}=\mathrm{N}$ bond in closed structure leads to a complete breakup, so that the structure is not stable in presence of Li.

- Finally, the statistical analysis of the $\mathrm{Li}-\mathrm{O} / \mathrm{Li}-\mathrm{N}$ distances reveals that $\mathrm{Li}$ is coordinated predominantly to $\mathrm{O}$ in the closed configuration, while for the linear one a similar statistical trend of Li-O and Li-N bonds is observed.

\subsection{Energetic stability.}

The total energy of the systems in bulk state and as free molecules, for each oxidation state was calculated. Energetic diagram in gas-phase of the closed/linear structures is represented in Figure S39, for relaxed molecules bearing total charges $0,-1$ and -2 . The calculations show that the closed structure is not stable for -2 charge (i.e., after structural relaxation at -2 charge, the closed structure is transformed into the linear one). It can be concluded that in gas phase, the most stable structure is the structure with total charge $Q=-1$, while the presence of the second electron is destabilizing the structure.

The diagram for total energy of the bulk states with different amounts of Li atoms inserted in the structure (i.e. $0 \%, 50 \%$ and $100 \%$ of the total 16 atom/supercell) is given in Figure $\$ 40$. We note that the presence of Li leads to an energetic stabilization of the structures. If no $\mathrm{Li}$ atoms are present, the closed structure is more stable, while in presence of $100 \% \mathrm{Li}$ atoms the linear one is more stable. In the intermediate case (only half of $\mathrm{Li}$ atoms are insert) we found structures with energies that are close to each other (around $0.5 \mathrm{eV}$ difference).

To be noted that the results on energetic stability corroborate those on the volume cell calculations in presence of Li. Indeed, for the linear structure we found that in presence of $\mathrm{Li}$ the cell volume diminishes, indicating a more efficient packing, while the total energy of the system shows an important energetic stabilization in this case.

The overall energy difference per Li atom (difference between the most stable structure in the two redox states / molecule / $\mathrm{Li}$ atom) is $\mathbf{0 . 6 7 / 0 . 7 2 ~} \mathbf{~ e V}$ for models with experimental / relaxed cell parameters, respectively. 


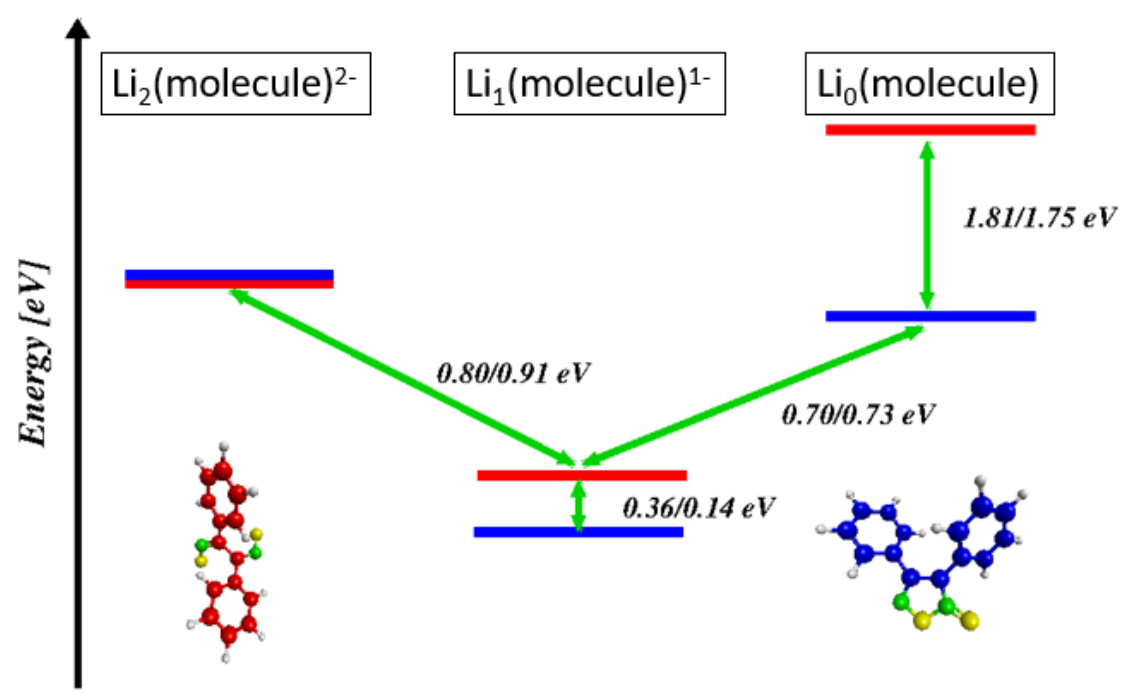

Supplementary Figure S39: Diagram of total energy/molecule for various oxidation states (total charge: $0,-1$ and -2 corresponding to $\mathrm{Li}_{0}$ (molecule)=[DPODO], $\mathrm{Li}_{1}(\text { molecule })^{1-}=$ [ $\mathrm{Li}_{1-}{ }^{-}$ 'DPGO'] and $\mathrm{Li}_{2}$ (molecule) ${ }^{2-}=\left[\mathrm{Li}_{2}-\mathrm{DPGO}\right]$, respectively) for linear/closed structures (represented with red/blue lines) in gas phase. The differences (expressed in eV) are indicated for two types of exchange-correlation functionals, M11 and M15, respectively.

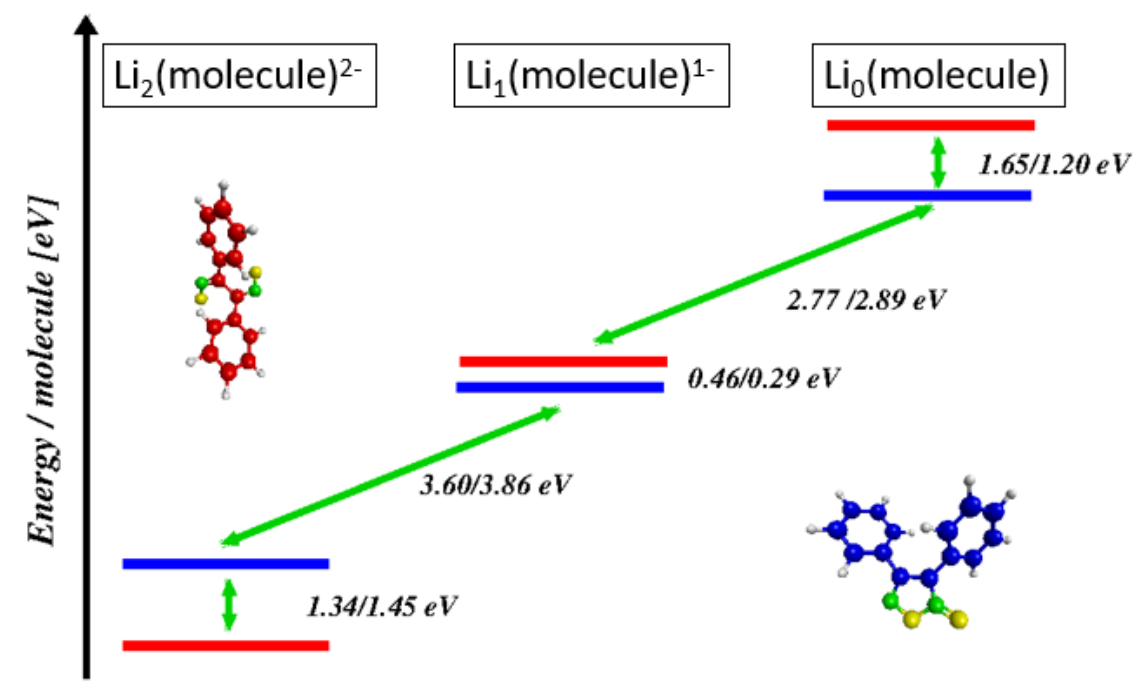

Supplementary Figure S40: Diagram of the total energy/unit cell (i.e. 8 molecules) for various oxidation states (amount of Li per molecule formulation: Lio(molecule)=[DPODO], $\mathrm{Li}_{1}$ (molecule $^{1-}=\left[\mathrm{Li}_{1} \text {-'DPGO'}\right]^{\prime}$ and $\mathrm{Li}_{2}$ (molecule $\left.^{2-}=\left[\mathrm{Li}_{2}-\mathrm{DPGO}\right]\right)$ for linear/closed structures (represented with red/blue lines) in bulk phase. The differences (expressed in eV) are indicated for two types of calculations: based on the experimental cell parameters / calculated cell parameters (see Tables S7 and S8), while atomic positions are fully relaxed in all cases. 


\subsection{Summary on the energetic properties:}

- Gas phase calculations indicate that a charge -2 leads to an energetic destabilization of the molecule. The closed structure does not exist in this state (transforms into a distorted linear configuration).

- In bulk form both types of structures (closed and linear) can accommodate one or two Li atoms per molecule and this is increasing their energetic stability. However, we mention that the molecular units of the closed structure are transformed to the linear structure in presence of Li atoms (i.e. similar to gas phase).

- Main difference between gas phase and bulk structure is that in bulk the presence of $\mathrm{Li}$ (i.e. ionized molecules) leads the most energetically stable structure, while for gas the molecule with charge -2 represents the less stable structure.

- We conclude that this is entirely a consequence of intermolecular interactions in bulk phase. Consequently, the redox potential should be depicted as strongly dependent on the intermolecular interactions in bulk.

\section{Conclusion}

DFT calculations prove a close correlation between the molecular conformation of $\mathrm{Li}_{2}$-DPGO $\rightleftarrows D P O D O$ (i.e. closed vs. linear) and the oxidation state. In the gas-phase, the closed structure is not stable in -2 charge state (i.e. a distorted linear structure is formed); in the bulk structures, while the configuration is still of linear in the presence of two Li atoms, the system is energetically stabilized, pointing out the role of intermolecular interactions as function of the redox state. The DFT results also indicate an energy barrier that can be estimated around $1 \mathrm{eV}$ between reduced and oxidized states. All results are consistent with the model presented and discussed in Figure S29 and experimental observations. 


\section{Bibliography}

1. Agilent, CrysAlisPro Version 1.171.37.35, Agilent Technologies UK Ltd, Yarnton, England. 2014.

2. Sheldrick, G. M., A short history of SHELX. Acta Crystallogr A 2008, 64 (Pt 1), 112-22.

3. Samsonov, V. A., Volodarskii, L.V. \& Khisamutdinov, G.K., Formation of 1,1,4,4-tetramethoxy-2,3,5,6tetrahydroximinocyclohexane by the interaction of trinitrosophloroglucinol with hydroxylamine hydrochloride in methanol. Chemistry of Heterocyclic Compounds 1997, 33, 471-474.

4. Joseph H. Trepagnier, W., Del., and John V. Vaughen, Woodstown, N. J. Preparation of dinitrosobenzenes (U.S. Patent No. 2,419,976). 1947.

5. Altomare, A.; Cuocci, C.; Giacovazzo, C.; Moliterni, A.; Rizzi, R.; Corriero, N.; Falcicchio, A., EXPO2013: a kit of tools for phasing crystal structures from powder data. Journal of Applied Crystallography 2013,46 (4), 1231-1235.

6. Kariuki, B., International Union of Crystallography - Extended Software/Methods Development Issue.

7. Poizot, P.; Gaubicher, J.; Renault, S.; Dubois, L.; Liang, Y.; Yao, Y., Opportunities and Challenges for Organic Electrodes in Electrochemical Energy Storage. Chem Rev 2020, 120 (14), 6490-6557.

8. Lakraychi, A. E.; Dolhem, F.; Vlad, A.; Becuwe, M., Organic Negative Electrode Materials for Metal-lon and Molecular-Ion Batteries: Progress and Challenges from a Molecular Engineering Perspective. Advanced Energy Materials 2021,11 (32).

9. $\quad$ Sieuw, L.; Lakraychi, A. E.; Rambabu, D.; Robeyns, K.; Jouhara, A.; Borodi, G.; Morari, C.; Poizot, P.; Vlad, A., Through-Space Charge Modulation Overriding Substituent Effect: Rise of the Redox Potential at $3.35 \mathrm{~V}$ in a Lithium-Phenolate Stereoelectronic Isomer. Chemistry of Materials 2020, 32 (23), 9996-10006.

10. Sieuw, L.; Jouhara, A.; Quarez, E.; Auger, C.; Gohy, J. F.; Poizot, P.; Vlad, A., A H-bond stabilized quinone electrode material for Li-organic batteries: the strength of weak bonds. Chem Sci 2019, 10 (2), 418-426.

11. Sillitoe, A. K. M. M. H., 3,4-Diphenylfurazan N-oxide. Acta Crystallographica Section B: Structural Crystallography and Crystal Chemistry 1978, 34 (6), 2021-2023.

12. Feuer, H., The Chemistry of the Nitro and Nitroso Groups, Part 1. Interscience: 1969.

13. Michael W. Schmidt, K. K. B., Jerry A. Boatz,Steven T. Elbert,Mark S. Gordon,Jan H. Jensen,Shiro Koseki,Nikita Matsunaga,Kiet A. Nguyen,Shujun Su,Theresa L. Windus,Michel Dupuis,John A. Montgomery Jr, General atomic and molecular electronic structure system. Journal of Computational Chemistry 1993, 14 (11), 137-1363.

14. Peverati, R.; Truhlar, D. G., Improving the Accuracy of Hybrid Meta-GGA Density Functionals by Range Separation. The Journal of Physical Chemistry Letters 2011, 2 (21), 2810-2817.

15. Ordejón, P.; Artacho, E.; Soler, J. M., Self-consistent order-N density-functional calculations for very large systems. Physical Review B 1996, 53 (16), R10441-R10444.

16. Perdew, J. P.; Ruzsinszky, A.; Csonka, G. I.; Vydrov, O. A.; Scuseria, G. E.; Constantin, L. A.; Zhou, X.; Burke, K., Restoring the Density-Gradient Expansion for Exchange in Solids and Surfaces. Physical Review Letters 2008, 100 (13), 136406.

17. Quertinmont, J.; Carletta, A.; Tumanov, N. A.; Leyssens, T.; Wouters, J.; Champagne, B., Assessing Density Functional Theory Approaches for Predicting the Structure and Relative Energy of Salicylideneaniline Molecular Switches in the Solid State. The Journal of Physical Chemistry C 2017, 121 (12), 6898-6908.

18. Grimme, S., Semiempirical GGA-type density functional constructed with a long-range dispersion correction. J Comput Chem 2006, 27 (15), 1787-99. 